\title{
STATIONARY GAUSSIAN RANDOM FIELDS ON HYPERBOLIC SPACES AND ON EUCLIDEAN SPHERES *,**
}

\author{
S. COHEN $^{1}$ AND M.A. LifShits ${ }^{2}$
}

\begin{abstract}
We recall necessary notions about the geometry and harmonic analysis on a hyperbolic space and provide lecture notes about homogeneous random functions parameterized by this space. The general principles are illustrated by construction of numerous examples analogous to Euclidean case. We also give a brief survey of the fields parameterized by Euclidean spheres. At the end we give a list of important open questions in hyperbolic case.
\end{abstract}

Mathematics Subject Classification. 60G15, 60G10, 51M10.

Received July 6, 2009.

\section{INTRODUCTION}

In this article we would like to give a self-contained introduction to spectral theory of stationary fields parameterized by hyperbolic spaces. This aim might look surprising for at least two reasons: first, the analytic theory of such fields is a classical part of harmonic analysis on symmetric spaces and complete references for this are $[9,12]$; second, hyperbolic spaces are not familiar to many probabilists.

Let us give probabilistic motivations for this study.

Fractional fields parameterized by finite-dimensional Euclidean spaces are today very often used in the modelling of images. See for instance [20] or $[3,8,13]$. Besides a lot of various applications, those fields can be used to obtain "textures" on flat spaces, but it is obvious even for non-specialist that "textures" exist on curved spaces. The most popular model for fractional fields is certainly the fractional Brownian field, and, maybe, even before the fractional Brownian fields, the Lévy Brownian field which will be rigorously defined in Section 8. These fields have stationary increments, which means that their distributions are invariant in a certain sense with respect to the action of the group of translations on the Euclidean space. Hence from the beginning it is quite obvious that among all manifolds that could parameterize the fields modelling "textures" on curved spaces, those on which a group of isometries is acting will be nicer than the others. A deeper reason is the fact that harmonic analysis is a classical topic on symmetric spaces (i.e. heuristically Riemannian manifolds on which isometries are acting nicely, see [16] for reference on this subject) and will provide us with a spectral analysis

Keywords and phrases. Hyperbolic space, Random fields, Lévy's Brownian field.

* ANR GDSA and grants NSh-638.2008.1, RFBR 09 - 01 - 12180-ofi_m.

** Most of this work was done when M. Lifshits was invited professor in Université Paul Sabatier.

1 Université de Toulouse, Université Paul Sabatier, Institut de Mathématiques de Toulouse, 31062 Toulouse, France. Serge.Cohen@math. univ-toulouse.fr

2 Department of Mathematics and Mechanics, St. Petersburg State University, Bibliotechnaya pl., 2, 198504, Stary Peterhof, Russia. lifts@mail.rcom.ru 
of these fields. However real symmetric spaces include Euclidean spaces, Euclidean spheres, and hyperbolic spaces, so why should we focus on hyperbolic spaces, when we want to extend the theory of stationary fields to curved spaces? Basically because the hyperbolic space is much more similar to the Euclidean spaces than the sphere for topological reason: it is not compact. This fundamental fact has a consequence for spectrum of the Laplacian which is continuous in Euclidean and hyperbolic case but discrete for the sphere.

See [1] for a reference on the geometry of hyperbolic spaces.

The case of stationary fields on Euclidean sphere will be addressed in Section 12 and turns out to be simpler than the hyperbolic case.

Working on this topic for probabilists and statisticians, no acquainted with geometry is not an easy job : there are a lot of notations to learn. Hence, although it would be natural to extend this study to general symmetric spaces, we chose to restrict ourselves to hyperbolic space and Euclidean sphere, to give a short introduction to useful tools we need in hyperbolic and spherical geometry. On the other side, analysts might think that harmonic analysis on symmetric space is an old subject and there is nothing new in this probabilistic treatment. They might be true, but we were surprised to discover that it was not easy at all to associate probabilistic fields to analytical objects like their covariance or the variance of their increments. As far as we know, the new part of this article is the integral representation of various canonical stationary fields. We hope that the comparison between the Euclidean case and the hyperbolic case would be of some interest and will help the reader to better understand stationary fields on curved spaces.

Just to begin with, here is one surprising fact about fractional Brownian fields on hyperbolic spaces and on the sphere: they just do exist not over the whole classical range of Hurst exponent $0<H<1$ as in the Euclidean case, but only when $0<H \leq 1 / 2$. See e.g. [20] on the range of admissible $H$ and more generally for simulations or applications in statistic. From the point of view of applications, it is a disturbing fact since $H$ is related not only to spectral properties of fractional fields but also to the regularity of the sample paths. More precisely, on Euclidean space, $H$-fractional Brownian fields have $H$-Hölder sample paths and the Hölder exponent is linked to the smoothness of "textures". Therefore there exists a gap for "textures" on sphere and hyperbolic spaces, they miss a canonical fractional model with stationary increments. At the beginning of this work, we hoped that integral representations of fractional Brownian field, or Lévy Brownian field, i.e. $H=1 / 2$ could be the starting point to define a canonical extension of fractional Brownian field to the case $H>1 / 2$. Unfortunately, by now we only have very partial answers for integral representations of fractional Brownian field and leave many open questions.

In Section 2 we provide the reader with a reminder of the Euclidean case for sake of comparison.

Section 3 is devoted to the introduction of the disk model of the hyperbolic space in dimension $n=2$. We recall the metric, the group of isometries, horocycles and the Laplace-Beltrami operator in this case. Actually we are mainly interested in the eigenfunctions of the Laplace-Beltrami operator which will be the basic tools for the Fourier transform on hyperbolic space. Radial eigenfunctions are called spherical functions.

In the following Section 4 the hyperboloid model for the hyperbolic space is introduced for all $n \in \mathbb{N}$ and related to the disk model when $n=2$. These two sections are elementary but we believe that they will be useful for probabilists.

A very important difference between hyperbolic and Euclidean space is the fact that in the first case there exist a lower part of the spectrum and an upper part of the spectrum. While the upper spectrum looks familiar to probabilists, because it is related to the Fourier transform on hyperbolic space, the lower spectrum has no counterpart in the Euclidean case and we provide series and integral representation of the fields corresponding to the lower spectrum in Section 5.

Sections 6 and 7 use a classical technique to generate stationary fields that goes back to Chentsov [7]. Let us consider a class $\mathcal{C}$ of geometric objects stable with respect to the action of isometries. If one knows how the isometry is acting on the class $\mathcal{C}$ and possesses a measure on $\mathcal{C}$ invariant under the action of the isometries, one can construct a white noise on $\mathcal{C}$ and a family of stationary fields on the underlying space associated to convolutions of $L^{2}$ functions with this white noise. For instance, for the class of geodesics of the hyperbolic plane, the stationary fields thus constructed include the Lévy Brownian field. Unfortunately, we do not know how to 
deduce the spectral decomposition for homogeneous field based on geodesics. However one can also consider the class of horocycles, and we get the spectral decomposition for homogeneous field based on horocycles in Section 6. It is a striking fact that this decomposition uses only the upper spectrum of Laplacian.

In Section 8 we compile all we know about the spectrum of fractional Brownian field. Although the description is partial, this part yields an explicit formula for the classical Fourier transform of the upper spectrum of Lévy Brownian field.

In the Euclidean case Ornstein-Uhlenbeck fields are classical stationary fields. We investigate in Section 9 the differences between Ornstein-Uhlenbeck fields on hyperbolic space and on Euclidean space.

Beside fractional Brownian fields, there exists another canonical field with stationary increments in the Euclidean space for which the variance of the increment is the square of the Euclidean norm. It is a degenerate field because it can be generated by a primitive white noise of rank $n$, where $n$ is the dimension of the Euclidean space. In the hyperbolic case there also exists a quadratic form in a generalized sense due to Gangolli [12], which is the variance of the increments of a stationary field on the hyperbolic space. In Section 10 we study properties of this field, which is generated by an infinite-dimensional white noise, and hence is no more degenerate.

The spectral properties of the white noise on hyperbolic space itself are studied in Section 11.

Section 12 is devoted to a short review of the stationary fields on Euclidean spheres.

Open questions are raised in Section 13.

\section{A sUmmary FOR the EUCLIDEAN CASE}

Let us recall definitions of stationary fields and fields with stationary increments on Euclidean space. Proofs of these elementary facts can be found in the general reference [25] for Gaussian fields parameterized by Euclidean spaces.

Definition 2.1. A centered Gaussian field $(X(t))_{t \in \mathbb{R}^{n}}$ is stationary if there exists a function $K$ such that the covariance $\mathcal{R}(t, s) \stackrel{\text { def }}{=} \mathbb{E} X_{t} \overline{X_{s}}=K(t-s)$. The function $K$ is called the auto-covariance function of the field $X$.

This definition will be generalized to fields parameterized by a space on which a group is operating in Definition 5.1. Definition 2.1 is a particular instance of Definition 5.1, when the group of translations is acting on $\mathbb{R}^{n}$.

Next, it is easy to prove that if $K$ is the auto-covariance function of stationary centered Gaussian field $X$ then it is a non-negative definite function in the following sense.

Definition 2.2. A real valued function $f$ on $\mathbb{R}^{n}$ is called non-negative definite if for every $d \geq 1, \lambda_{1}, \ldots, \lambda_{d} \in \mathbb{C}$, $t_{1}, \ldots, t_{d} \in \mathbb{R}^{n}$

$$
\sum_{i, j=1}^{d} \lambda_{i} \overline{\lambda_{j}} f\left(t_{i}-t_{j}\right) \geq 0 .
$$

This definition will be generalized in Section 5.1.

Moreover, if $K$ is a non-negative definite function then there exists a stationary centered Gaussian field $X$ such that $K$ is the auto-covariance function of the field $X$.

One can show that distributions of stationary centered Gaussian field $X$ satisfy

$$
(X(t+\delta))_{t \in \mathbb{R}^{n}} \stackrel{(d)}{=}(X(t))_{t \in \mathbb{R}^{n}}, \quad \forall \delta \in \mathbb{R}^{n} .
$$

This formula simply means that the distribution of the field is invariant under the action of the translations.

We also recall a spectral characterization of non-negative definite functions called Bochner's theorem (see [33] for a proof).

Theorem 2.3. Bochner's theorem.

Among the continuous real valued functions on $\mathbb{R}^{n}$, the non-negative definite functions are those functions that are the Fourier transforms of finite measures. 
Definition 2.4. If $X$ is a stationary centered Gaussian field such that the auto-covariance function $K$ is continuous, by Bochner Theorem 2.3, there exists a unique finite measure $\mu$ on $\mathbb{R}^{n}$ such that

$$
K(t)=\int_{\mathbb{R}^{n}} \mathrm{e}^{\mathrm{i} t . \xi} \mu(\mathrm{d} \xi)
$$

The measure $\mu$ is called the spectral measure of the field $X$. If $\mu$ admits a density with respect to the Lebesgue measure $\mathrm{d} \xi$, this density is called the spectral density of $X$.

Spectral representation of Gaussian stationary field.

Let $X_{t}, t \in \mathbb{R}^{n}$ be a centered stationary Gaussian field with auto-covariance

$$
K(h)=\mathbb{E} X_{t+h} X_{t} .
$$

Assume that $K$ is a continuous function and that the corresponding spectral measure $\mu(\mathrm{d} \xi)=f(\xi) \mathrm{d} \xi$. Notice that $K(h)=K(-h)$, and by Bochner's theorem $K(h)=\int_{\mathbb{R}^{n}} \mathrm{e}^{\mathrm{i} h \cdot \xi} f(\xi) \mathrm{d} \xi$, so $f(\xi)=f(-\xi)$ is a non negative function in $L^{1}\left(\mathbb{R}^{n}\right)$. Let us consider the field defined by

$$
Y_{t}=\int_{\mathbb{R}^{n}} \sqrt{f(\xi)} \mathrm{e}^{\mathrm{i} t \cdot \xi} \widehat{W}(\mathrm{~d} \xi),
$$

where $\widehat{W}(\mathrm{~d} \xi)$ is an appropriate complex white noise controlled by Lebesgue measure (we refer to [25] for the definition and basic properties of the white noise).

Clearly, $Y$ is a centered Gaussian field with auto-covariance function

$$
\begin{aligned}
\mathbb{E} Y_{t} Y_{s} & =\int_{\mathbb{R}^{n}} f(\xi) \mathrm{e}^{\mathrm{i}(t-s) \xi} \mathrm{d} \xi \\
& =K(t-s) .
\end{aligned}
$$

Hence, $Y$ and $X$ have the same distribution and (2.3) is called the spectral representation of $X$.

Let us consider an important sub-class of stationary fields. Namely, let us make an additional assumption that the distribution of a stationary field $X$ is rotation-invariant. It means that the measure $\mu$ in $(2.2)$ must be spherically invariant. Hence, (2.2) writes as

$$
K(t)=\int_{0}^{\infty} \int_{\mathbb{S}^{n-1}} \mathrm{e}^{\mathrm{i} t \cdot \lambda \sigma} \mathrm{d} \sigma \nu(\mathrm{d} \lambda),
$$

where $\mathrm{d} \sigma$ is the unique rotation-invariant unit measure on the sphere $\mathbb{S}^{n-1}$ and $\nu$ is a finite measure on $(0, \infty)$.

Definition 2.5. The functions

$$
\phi_{\lambda}(t):=\int_{\mathbb{S}^{n-1}} \mathrm{e}^{\mathrm{i} t \cdot \lambda \sigma} \mathrm{d} \sigma, \quad t \in \mathbb{R}^{n}
$$

are called spherical functions on $\mathbb{R}^{n}$.

With this definition the previous relation writes simply as

$$
K(t)=\int_{0}^{\infty} \phi_{\lambda}(t) \nu(\mathrm{d} \lambda)
$$

It means that any rotation invariant covariance of a stationary field is just a mixture of spherical functions. This also explains the importance of the latter notion. 
Let us mention few properties of spherical functions. It is almost obvious that their values are real and that $\phi_{\lambda}(t)$ is actually determined by $\|t\|$. There is a useful self-similarity property

$$
\phi_{\lambda}(c t)=\phi_{c \lambda}(t), \quad t \in \mathbb{R}^{n}, c>0 .
$$

and even more important relation to Laplace operator. Namely, an easy calculation yields

$$
\Delta \phi_{\lambda}(t)=-\lambda^{2} \phi_{\lambda}(t) .
$$

Hence, $\phi_{\lambda}$ are spherically invariant eigenfunctions of Laplace operator that have negative eigenvalues. This fact will help us to identify the spherical functions when we will move from $\mathbb{R}^{n}$ to other manifolds.

Let us now consider fields with stationary increments.

Definition 2.6. A field $(X(t))_{t \in \mathbb{R}^{n}}$ such that

$$
(X(t+\delta)-X(s+\delta))_{t \in \mathbb{R}^{n}} \stackrel{(d)}{=}(X(t)-X(s))_{t \in \mathbb{R}^{n}},
$$

for every $s, t$, and $\delta$ in $\mathbb{R}^{n}$ is called a field with stationary increments.

There exists also a spectral representation of the covariance of fields with stationary increments, which is similar to Bochner's theorem. Let us assume that $X$ is a centered Gaussian field with stationary increments. Moreover, if $X_{0}=0$ a.s. and the covariance is continuous, then there exists a sigma-finite measure $\mu$ on $\mathbb{R}^{n}$ satisfying

$$
\int_{\mathbb{R}^{n}} \min \left(1,\|\xi\|^{2}\right) \mu(\mathrm{d} \xi)<+\infty
$$

and a symmetric non-negative definite matrix $\Sigma$ such that

$$
\mathcal{R}(t, s)=\int_{\mathbb{R}^{n}}\left(\mathrm{e}^{\mathrm{i} t \cdot \xi}-1\right)\left(\mathrm{e}^{-\mathrm{i} s \cdot \xi}-1\right) \mu(\mathrm{d} \xi)+t \cdot \Sigma s .
$$

(See a discussion concerning this result in [40] and the references therein.) On can also deduce from this result an integral representation for Gaussian fields with stationary increments.

Let us consider a centered Gaussian field $X$ with stationary increments such that $X_{0}=0$ a.s. Let us assume that its covariance function is continuous, and that the spectral measure $\mu(\mathrm{d} \xi)=f(\xi) \mathrm{d} \xi$. As in the stationary case, $f$ is called the spectral density of $X$. Then

$$
X_{t} \stackrel{(d)}{=} \int_{\mathbb{R}^{n}}\left(\mathrm{e}^{\mathrm{i} t \cdot \xi}-1\right) \sqrt{f(\xi)} \widehat{W}(\mathrm{~d} \xi)+t \cdot N
$$

where $\widehat{W}(\mathrm{~d} \xi)$ is the white noise controlled by Lebesgue measure and where $N$ is a centered Gaussian random vector with covariance $\Sigma$ independent of $\widehat{W}$. Note that $\int_{\mathbb{R}^{n}}\left(\mathrm{e}^{\mathrm{i} t \cdot \xi}-1\right) \sqrt{f(\xi)} \widehat{W}(\mathrm{~d} \xi)$ and $N$ are independent for all $t$.

Again, let us consider a sub-class of rotation invariant fields with stationary increments. In this case the spectral measure $\mu$ in (2.6) is spherically invariant and $\Sigma$ is the unit matrix up to a positive constant. Therefore, (2.6) writes as

$$
\mathcal{R}(t, s)=\int_{0}^{\infty} \int_{\mathbb{S}^{n-1}}\left(\mathrm{e}^{\mathrm{i} t \cdot \lambda \sigma}-1\right)\left(\mathrm{e}^{-\mathrm{i} s \cdot \lambda \sigma}-1\right) \mathrm{d} \sigma \mathrm{d} \nu(\lambda)+a t \cdot s,
$$

where $a \geq 0$ and $\nu$ is a measure on $(0, \infty)$ satisfying

$$
\int_{0}^{\infty} \min \left(1, \lambda^{2}\right) \nu(\mathrm{d} \lambda)<+\infty
$$


Actually, all properties of $X$ are defined by the structure function

$$
\begin{aligned}
\mathcal{R}(t, t) & =\int_{0}^{\infty} \int_{\mathbb{S}^{n-1}}\left|\mathrm{e}^{\mathrm{i} t \cdot \lambda \sigma}-1\right|^{2} \mathrm{~d} \sigma \nu(\mathrm{d} \lambda)+a\|t\|^{2} \\
& =2 \int_{0}^{\infty}\left(1-\phi_{\lambda}(t)\right) \nu(\mathrm{d} \lambda)+a\|t\|^{2} .
\end{aligned}
$$

Notice that the quadratic term is directly related to the behavior of spherical functions with small parameter $\lambda$. Namely, by expanding the exponential function we readily obtain

$$
\lim _{\lambda \rightarrow 0} \frac{1-\phi_{\lambda}(t)}{\lambda^{2}}=\frac{\|t\|^{2}}{2 n} .
$$

Two important examples could be mentioned at this stage. First, let $X_{1}, \ldots, X_{n}$ be a sequence of i.i.d. standard normal random variables. Let a finite rank field with stationary increments $(X(t))_{t \in \mathbb{R}^{n}}$, be defined by

$$
X(t)=\sum_{j=1}^{n} t_{j} X_{j} .
$$

Then $\mathcal{R}(t, t)=\sum_{j=1}^{n} t_{j}^{2}=\|t\|^{2}$ which clearly corresponds to the quadratic term in the expression for the structure function. Notice that in our future investigation the similar generalized quadratic terms will appear and they will be still related to the behavior of the spherical function of small parameter. However, the corresponding random fields will not be so degenerate as $X$.

Second, the formula

$$
\mathcal{R}(t, t)=\|t\|^{2 H}, \quad 0<H<1,
$$

defines a family of rotationally invariant Gaussian fields with stationary increments $W^{H}$ called $H$-fractional Lévy Brownian fields. The classical case $H=1 / 2$ was considered by Lévy [22]. By using property (2.4), it is easy to see that the spectral measures

$$
\nu_{H}(\mathrm{~d} \lambda)=\frac{c_{H, n} \mathrm{~d} \lambda}{\lambda^{2 H+1}}, \quad 0<\lambda<\infty
$$

with appropriate choice of constants $c_{H, n}$, correspond to $W^{H}$. Notice that $\nu_{H}$ satisfies the integrability condition (2.8) exactly when $H \in(0,1)$.

\section{Disk MODEL OF HYPERBolid SPACE}

\subsection{The space and its transformations}

In this section we only consider the real hyperbolic space of dimension 2. The disk model (or Poincaré model) for this space is a unit disk $\mathbb{D}=\{z \in \mathbb{C},|z|<1\}$ on the complex plane. The space can be endowed with a Riemannian structure, from which we only need, so far, the distance [17], p. 30,

$$
d\left(z_{1}, z_{2}\right):=\frac{1}{2} \log \frac{\left|1-\bar{z}_{1} z_{2}\right|+\left|z_{2}-z_{1}\right|}{\left|1-\bar{z}_{1} z_{2}\right|-\left|z_{2}-z_{1}\right|} .
$$

In particular,

$$
d(0, z):=\frac{1}{2} \log \frac{1+|z|}{1-|z|}, \quad z \in \mathbb{D}
$$

Conversely, if $d(0, z)=r$, then

$$
|z|=\frac{\mathrm{e}^{r}-\mathrm{e}^{-r}}{\mathrm{e}^{r}+\mathrm{e}^{-r}}=\tanh (r) .
$$


Consider a group of isometries indexed by the matrix group

$$
S U(1,1)=\left\{\left(\begin{array}{cc}
\alpha & \beta \\
\bar{\beta} & \bar{\alpha}
\end{array}\right):|\alpha|^{2}-|\beta|^{2}=1\right\} .
$$

Every $g \in S U(1,1)$ acts on $\mathbb{D}$ as

$$
g(z)=\frac{\alpha z+\beta}{\bar{\beta} z+\bar{\alpha}}
$$

and we identify the matrix $g$ with this mapping. One can show that $g$ is an isometry with respect to the distance $d$.

Consider some important subgroups of $S U(1,1)$.

Example 3.1. Subgroup of rotations

$$
S O(2)=\left\{\rho=\left(\begin{array}{cc}
\mathrm{e}^{\mathrm{i} \phi} & 0 \\
0 & \mathrm{e}^{-\mathrm{i} \phi}
\end{array}\right), 0 \leq \phi \leq 2 \pi\right\} .
$$

Of course, $\rho(z)=\mathrm{e}^{2 \mathrm{i} \phi} z$ is a simple rotation. It is obvious that $S O(2)=\{g: g(0)=0\}$. Moreover, if $g_{1}(0)=g_{2}(0)$, then there exists a rotation $\rho \in S O(2)$ such that $g_{1}=g_{2} \rho$. In other words, $\mathbb{D}$ can be identified with the quotient space $S U(1,1) / S O(2)$ and thus considered as a symmetric space. It is worthwhile to mention that more generally all symmetric spaces are isomorphic to the group $G$ of their isometries quotioned by the subgroup of isometries that fix a particular point, denoted by $K$. In the case of $\mathbb{D}, K=S O(2)$.

Example 3.2. Translation subgroup

$$
A=\left\{a_{t}=\left(\begin{array}{c}
\cosh (t) \sinh (t) \\
\sinh (t) \\
\cosh (t)
\end{array}\right),-\infty<t<\infty\right\} .
$$

Since $a_{t}$ is a real matrix, it maps the diameter $(-1,+1)$ onto itself. An easy calculation shows the translation property

where we let

$$
\tilde{d}\left(0, a_{t} z\right)=\tilde{d}(0, z)+t, \quad z \in(-1,+1)
$$

$$
\tilde{d}(0, z):=\left\{\begin{array}{cl}
d(0, z), & 0 \leq z<1, \\
-d(0, z), & -1<z \leq 0 .
\end{array}\right.
$$

Example 3.3. Orthogonal shift subgroup

$$
N=\left\{n_{u}=\left(\begin{array}{cc}
1+i u & -i u \\
i u & 1-i u
\end{array}\right),-\infty<u<\infty\right\} .
$$

Note that any $g \in S U(1,1)$ also acts on the boundary $B=\{z \in \mathbb{C},|z|=1\}$ of $\mathbb{D}$. Indeed, we have $g(1)=\frac{\alpha+\beta}{\beta+\bar{\alpha}}$, hence $|g(1)|=1$. Next, remark that any element of $B$ writes as $\rho(1)$ for some rotation $\rho \in S U(1,1)$. Finally, we have $g(\rho(1))=(g \rho)(1) \in B$.

Any point in $\mathbb{D}$ can be achieved from 0 by using a unique combination of shifts $z=n_{u} a_{t}(0)$. Therefore, one can consider $(u, t)$ as coordinates of $z$ called Iwasawa coordinates, see Figure 1.

One can establish commutation rule [17], p. 36, $n_{u} a_{t}=a_{t} n_{u \mathrm{e}^{-2 t}}$.

Actually there are other coordinates on $\mathbb{D}$, and we fix some notation:

- Euclidean coordinates $z=x+\mathrm{i} y$,

- polar Euclidean coordinates $z=\rho \mathrm{e}^{\mathrm{i} \theta}$ with $\theta \in[0,2 \pi)$,

- polar hyperbolic coordinates $z=\tanh (r) \mathrm{e}^{\mathrm{i} \theta}$, where $r=d(z, 0)$ as deduced from (3.3). 


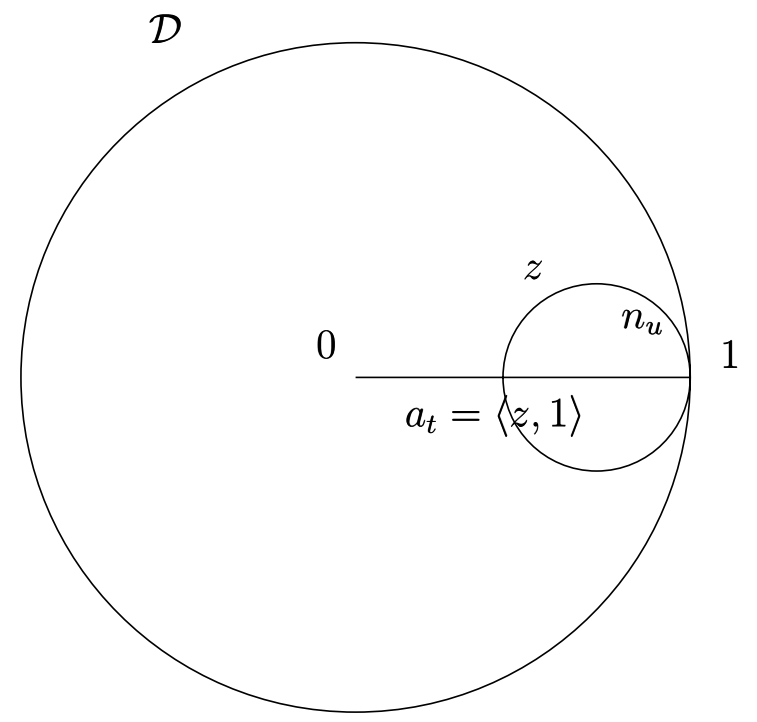

FiguRE 1. Horocycle.

\subsection{Invariant measure}

There exists an infinite measure which is invariant with respect to all $g \in S U(1,1)$. It is unique up to a constant that we denote by the same $c$ in the following formula. In Euclidean coordinates this measure writes as

$$
\mathrm{d} z=c\left(1-x^{2}-y^{2}\right)^{-2} \mathrm{~d} x \mathrm{~d} y=\frac{c|z|}{\left(1-|z|^{2}\right)^{2}} \mathrm{~d}|z| \mathrm{d} \theta .
$$

By (3.3), we can write in polar hyperbolic coordinates

$$
\mathrm{d} z=c \sinh (2 r) \mathrm{d} r \mathrm{~d} \theta .
$$

It follows easily that the measure of the ball of radius $r$ (in hyperbolic distance) is $V(r)=c \cosh ^{2}(r)$. On the other hand, in Iwasawa coordinates [17], p. 37

$$
\mathrm{d} z=c \mathrm{e}^{-2 t} \mathrm{~d} u \mathrm{~d} t
$$

\subsection{Laplace-Beltrami operator}

One may wonder why we chose the distance $d$ in (3.1) on $\mathbb{D}$. Actually $\mathbb{D}$ is a Riemannian manifold and $d$ is very natural from this point of view, as a Riemann distance.

On any Riemannian manifold there exists a Laplace-Beltrami operator, which is of particular interest for spectral studies. For Euclidean space, Laplace-Beltrami operator is just the classical Laplace operator. Let us recall that Laplace-Beltrami operator on hyperbolic plane [17], p. 38, acts on smooth functions in polar hyperbolic coordinates $f=f(r, \theta)$ on $\mathbb{D}$ as

$$
\Delta f=\frac{\mathrm{d}^{2}}{\mathrm{~d} r^{2}} f+2 \operatorname{coth}(2 r) \frac{\mathrm{d}}{\mathrm{d} r} f+\frac{4}{\sinh ^{2}(2 r)} \frac{\mathrm{d}^{2}}{\mathrm{~d} \theta^{2}} f
$$

where $r$ denotes hyperbolic distance from 0 . In particular, for spherically invariant functions, $f=f(r)$,

$$
\Delta f=\frac{\mathrm{d}^{2}}{\mathrm{~d} r^{2}} f+2 \operatorname{coth}(2 r) \frac{\mathrm{d}}{\mathrm{d} r} f .
$$




\subsection{Horocycles and the outer product}

We now introduce an outer product $\langle z, b\rangle$ for $z \in \mathbb{D}, b \in B$. In some respects it is similar to the scalar product in Euclidean space.

Let us be more precise and recall that $B$ denotes the boundary of the disk $\mathbb{D}$. Any Euclidean circle touching $B$ and belonging to $\mathbb{D}$ except for this touching point is called horocycle. We will denote $\mathcal{H}$ the two-dimensional space of all horocycles.

For any $b \in B$ and $z \in \mathbb{D}$ consider the unique horocycle $h$ that contains both $b$ and $z$. Denote the outer product

$$
\langle z, b\rangle:= \begin{cases}+\operatorname{dist}(0, h), & \text { if } 0 \text { is outside of } h, \\ -\operatorname{dist}(0, h), & \text { if } 0 \text { is inside of } h,\end{cases}
$$

where

$$
\operatorname{dist}(0, h)=\inf _{\zeta \in h} d(0, \zeta)
$$

More explicitly, [17], p. 34,

$$
\langle z, b\rangle=\frac{1}{2} \log \frac{1-|z|^{2}}{|z-b|^{2}} .
$$

All horocycles are the sets of the form $\{z \in \mathbb{D},\langle z, b\rangle=c\}$ thus providing a counterpart to hyperplanes in Euclidean space.

We start with some elementary properties of this outer product.

Proposition 3.4. We have the following.

- For all $b \in B$ it is true that $\langle 0, b\rangle=0$.

- For any rotation $\rho$ we have $\langle\rho(z), \rho(b)\rangle=\langle z, b\rangle$. In particular, $\langle-z,-b\rangle=\langle z, b\rangle$. However, in general we have $\langle-z, b\rangle \neq-\langle z, b\rangle$ and $\langle z,-b\rangle \neq-\langle z, b\rangle$. The corresponding equalities are only true when $z$ belongs to the diameter $(-b,+b)$.

- There is a "magic formula",

$$
\langle g(z), g(b)\rangle=\langle z, b\rangle+\langle g(0), g(b)\rangle .
$$

- We have

$$
\langle g(0), g(b)\rangle=-\left\langle g^{-1}(0), b\right\rangle .
$$

- Using (3.10), we can write the derivative of the boundary action [17], p. 45, as

$$
\frac{\mathrm{d} g(b)}{\mathrm{d} b}=\mathrm{e}^{2\left\langle g^{-1}(0), b\right\rangle}=\mathrm{e}^{-2\langle g(0), g(b)\rangle} .
$$

Proof of (3.9). Let $b=1$. Represent $g=\rho n_{u} a_{t}$. Then

$$
\begin{aligned}
\langle g(z), g(b)\rangle & =\left\langle\rho n_{u} a_{t}(z), \rho n_{u} a_{t}(b)\right\rangle=\left\langle n_{u} a_{t}(z), n_{u} a_{t}(b)\right\rangle \\
& =\left\langle n_{u} a_{t}(z), 1\right\rangle=\left\langle a_{t}(z), 1\right\rangle=\langle z, 1\rangle+t .
\end{aligned}
$$

Putting here $z=0$ we have $\langle g(0), g(b)\rangle=t$ and (3.9) holds. For general $b \in B$, we represent it as a result of rotation $b=\rho(1)$ and obtain

$$
\begin{aligned}
\langle g(z), g(b)\rangle & =\left\langle g \rho \rho^{-1}(z), g \rho(1)\right\rangle \\
& =\left\langle\rho^{-1}(z), 1\right\rangle+\langle g \rho(0), g \rho(1)\rangle=\langle z, b\rangle+\langle g(0), g(b)\rangle .
\end{aligned}
$$


Proof of (3.10). putting $z=g^{-1}(0)$ in (3.9) we get

$$
0=\langle 0, g(b)\rangle=\left\langle g g^{-1}(0), g(b)\right\rangle=\left\langle g^{-1}(0), b\right\rangle+\langle g(0), g(b)\rangle .
$$

Let now $h$ be a horocycle containing $b \in B$. Since the value $r=\langle z, b\rangle$ is the same for all $z \in h$, we can identify the horocycle $h$ with the pair $(r, b)$. Hence,

$$
\mathcal{H}=\{(r, b), r \in \mathbb{R}, b \in B\} .
$$

It is instructive to see that any $g \in S U(1,1)$ transforms horocycles in horocycles. Indeed, let $b=1$. Then, for any rotation $\rho \in S U(1,1)$ we have $\rho(r, 1)=(r, \rho(b))$, for any translation $a_{t}$ we have $a_{t}(r, 1)=(r+t, 1)$, and for any orthogonal shift $n_{u}$ we have $n_{u}(r, 1)=(r, 1)$.

Since any $g \in S U(1,1)$ can be represented ${ }^{3}$ as $g=\rho n_{u} a_{t}$, we get $g(1)=\rho(1)$,

$$
\langle g(0), g(1)\rangle=\left\langle\rho n_{u} a_{t}(0), \rho(1)\right\rangle=\left\langle n_{u} a_{t}(0), 1\right\rangle=\left\langle a_{t}(0), 1\right\rangle=t,
$$

hence

$$
\begin{aligned}
g(r, 1) & =\rho n_{u} a_{t}(r, 1)=\rho(r+t, 1)=(r+t, \rho(1)) \\
& =(r+\langle g(0), g(1)\rangle, g(1)) .
\end{aligned}
$$

More generally, we have

$$
g(r, b)=(r+\langle g(0), g(b)\rangle, g(b)), \quad \forall r \in \mathbb{R}, b \in B .
$$

This formula explains the action of $S U(1,1)$ on $\mathcal{H}$. It is now easy to establish the existence of the unique (up to a constant) invariant measure of $\mathcal{H}$ which is

$$
\mathrm{d} h=\mathrm{e}^{2 r} \mathrm{~d} r \mathrm{~d} b,
$$

where $\mathrm{d} b$ is the Haar measure on $B$.

We will need the following important result for the white noise representation of the random fields. The next formula is the counterpart in hyperbolic geometry of the classical functional equations for exponential functions in Euclidean geometry : $\mathrm{e}^{\mathrm{i} t \xi} \mathrm{e}^{\mathrm{i} s \xi}=\mathrm{e}^{\mathrm{i}(t+s) \xi}$.

Lemma 3.5. Let $g_{1}, g_{2} \in S U(1,1)$ and $\lambda \in \mathbb{R}$. Then

$$
\int_{B} \mathrm{e}^{(\mathrm{i} \lambda+1)\left\langle g_{1}(0), b\right\rangle} \overline{\mathrm{e}^{(\mathrm{i} \lambda+1)\left\langle g_{2}(0), b\right\rangle}} \mathrm{d} b=\int_{B} \mathrm{e}^{(\mathrm{i} \lambda+1)\left\langle g_{2}^{-1} g_{1}(0), b\right\rangle} \mathrm{d} b .
$$

Proof. Make the variable change $b=g_{2} y$ so that, according to (3.11),

$$
\mathrm{d} y=\exp \left\{2\left\langle g_{2}(0), b\right\rangle\right\} \mathrm{d} b .
$$

We see that the left hand side of the identity to be proved equals

$$
\int_{B} \mathrm{e}^{(\mathrm{i} \lambda+1)\left\langle g_{1}(0), b\right\rangle} \mathrm{e}^{-(\mathrm{i} \lambda+1)\left\langle g_{2}(0), b\right\rangle} \mathrm{d} y=\int_{B} \mathrm{e}^{(\mathrm{i} \lambda+1)\left(\left\langle g_{1}(0), b\right\rangle-\left\langle g_{2}(0), b\right\rangle\right)} \mathrm{d} y .
$$

\footnotetext{
${ }^{3}$ More precisely, find $u, t$ such that $g^{-1}(0)=n_{u} a_{t}(0)$, then there is a rotation $\rho$ such that $g^{-1}=n_{u} a_{t} \rho^{-1}$, hence $g=\rho a_{-t} n_{-u}$.
} 
Notice that by (3.9)

$$
\begin{aligned}
\left\langle g_{1}(0), b\right\rangle-\left\langle g_{2}(0), b\right\rangle & =\left\langle g_{1}(0), g_{2}(y)\right\rangle-\left\langle g_{2}(0), g_{2}(y)\right\rangle \\
& =\left\langle g_{2} g_{2}^{-1} g_{1}(0), g_{2}(y)\right\rangle-\left\langle g_{2}(0), g_{2}(y)\right\rangle=\left\langle g_{2}^{-1} g_{1}(0), y\right\rangle,
\end{aligned}
$$

as required.

Notice that

$$
\mathrm{e}^{2\langle z, b\rangle}=\frac{1-|z|^{2}}{|z-b|^{2}}
$$

is nothing but the classical Poisson kernel. Recall that for any harmonic function $F$ (i.e. $\Delta F=0$ ) on $\mathbb{D}$ one has

$$
F(z)=\int_{B} \mathrm{e}^{2\langle z, b\rangle} F(b) \mathrm{d} b .
$$

In particular, by letting $F \equiv 1$, we get

$$
\int_{B} \mathrm{e}^{2\langle z, b\rangle} \mathrm{d} b=1, \quad \forall z \in \mathbb{D} .
$$

One can also get the same formula by using Lemma 3.5 with $\lambda=0$ and $g_{1}(0)=g_{2}(0)=z$.

\subsection{Spherical functions}

For any $\lambda \in \mathbb{R}$ we define spherical functions (see [17], p. 38)

$$
\varphi_{\lambda}(z)=\int_{B} \mathrm{e}^{(\mathrm{i} \lambda+1)\langle z, b\rangle} \mathrm{d} b .
$$

Obviously, $\varphi_{\lambda}(0)=1$ and, we see that $\varphi_{\lambda}(\cdot)$ is rotation-invariant by rotation invariance of $\langle\cdot, \cdot\rangle$, cf. Proposition 3.4. It follows from (3.17) that $\left|\varphi_{\lambda}(z)\right| \leq 1$. We shall see later that $\varphi_{\lambda}(\cdot)$ is non-negative definite on $\mathbb{D}$.

Notice that $\varphi_{\lambda}(z)=\varphi_{-\lambda}(z)$. Indeed, let $g_{2}(0)=z, g_{1}=i d$ in (3.15). We see that

$$
\begin{aligned}
\varphi_{-\lambda}(z) & =\int_{B} \overline{\mathrm{e}^{(\mathrm{i} \lambda+1)\left\langle g_{2}(0), b\right\rangle}} \mathrm{d} b=\int_{B} \mathrm{e}^{(\mathrm{i} \lambda+1)\left\langle g_{2}^{-1}(0), b\right\rangle} \mathrm{d} b \\
& =\varphi_{\lambda}\left(g_{2}^{-1}(0)\right) .
\end{aligned}
$$

Next, since $g_{2}$ is an isometry, we have $d\left(g_{2}^{-1}(0), 0\right)=d\left(0, g_{2}(0)\right)$. It follows that there exists a rotation $\rho$ such that $\rho g_{2}^{-1}(0)=g_{2}(0)$. By rotation invariance of $\varphi_{\lambda}$, we obtain $\varphi_{\lambda}\left(g_{2}^{-1}(0)\right)=\varphi_{\lambda}\left(\rho g_{2}^{-1}(0)\right)=\varphi_{\lambda}\left(g_{2}(0)\right)=\varphi_{\lambda}(z)$, which concludes the computations.

Since obviously $\varphi_{-\lambda}=\overline{\varphi_{\lambda}}$, we can also conclude that the values of $\varphi_{\lambda}$ actually are real.

The importance of these functions comes from the fact that they are eigenfunctions of Laplace-Beltrami operator defined in (3.6). Namely,

$$
\Delta \varphi_{\lambda}=-\left(1+\lambda^{2}\right) \varphi_{\lambda}
$$

\subsection{Fourier transform}

At this point, we have introduced all we need to recall from [17] , p. 33 the Fourier transform on the hyperbolic disk, which is an isometry $f \mapsto \tilde{f}$

$$
L^{2}(\mathbb{D}) \rightarrow L^{2}\left(\mathbb{R} \times B, \frac{\lambda \tanh (\pi \lambda / 2)}{2 \pi} \mathrm{d} \lambda \mathrm{d} b\right)
$$


given (when the involved integrals are well defined) by

$$
\tilde{f}(\lambda, b)=\int_{\mathbb{D}} f(z) \mathrm{e}^{(-\mathrm{i} \lambda+1)\langle z, b\rangle} \mathrm{d} z
$$

and its inverse

$$
f(z)=\frac{1}{4 \pi} \int_{\mathbb{R}} \int_{B} \tilde{f}(\lambda, b) \mathrm{e}^{(\mathrm{i} \lambda+1)\langle z, b\rangle} \lambda \tanh (\pi \lambda / 2) \mathrm{d} b \mathrm{~d} \lambda .
$$

For any radial function $f$ the Fourier transform $\tilde{f}(\lambda, b)$ is also radial (does not depend on the argument $b$ ). Hence,

$$
\begin{aligned}
\tilde{f}(\lambda) & :=\tilde{f}(\lambda, b)=\int_{B} \tilde{f}(\lambda, b) \mathrm{d} b \\
& =\int_{B} \int_{\mathbb{D}} f(z) \mathrm{e}^{(-\mathrm{i} \lambda+1)\langle z, b\rangle} \mathrm{d} z \mathrm{~d} b=\int_{\mathbb{D}} f(z) \int_{B} \mathrm{e}^{(-\mathrm{i} \lambda+1)\langle z, b\rangle} \mathrm{d} b \mathrm{~d} z \\
& =\int_{\mathbb{D}} f(z) \varphi_{-\lambda}(z) \mathrm{d} z=\int_{\mathbb{D}} f(z) \varphi_{\lambda}(z) \mathrm{d} z .
\end{aligned}
$$

Inversion formula (3.21) yields

$$
\begin{aligned}
f(z) & =\frac{1}{4 \pi} \int_{B} \int_{\mathbb{R}} \tilde{f}(\lambda, b) \mathrm{e}^{(\mathrm{i} \lambda+1)\langle z, b\rangle} \lambda \tanh (\pi \lambda / 2) \mathrm{d} \lambda \mathrm{d} b \\
& =\frac{1}{4 \pi} \int_{\mathbb{R}} \tilde{f}(\lambda) \lambda \tanh (\pi \lambda / 2) \int_{B} \mathrm{e}^{(\mathrm{i} \lambda+1)\langle z, b\rangle} \mathrm{d} b \mathrm{~d} \lambda \\
& =\frac{1}{4 \pi} \int_{\mathbb{R}} \tilde{f}(\lambda) \lambda \tanh (\pi \lambda / 2) \varphi_{\lambda}(z) \mathrm{d} \lambda \\
& =\frac{1}{2 \pi} \int_{0}^{\infty} \tilde{f}(\lambda) \lambda \tanh (\pi \lambda / 2) \varphi_{\lambda}(z) \mathrm{d} \lambda .
\end{aligned}
$$

The strange tanh expression comes from the asymptotic behavior of spherical functions via Harish-Chandra function (see (4.33) below).

\section{Hyperboloid MODEL}

\subsection{Main objects}

In this section we consider the real $n$-dimensional hyperbolic space represented as a connected component of a hyperboloid in $\mathbb{R}^{n+1}$. For $n=2$, we show the connection with the disk model considered in Section 3 .

Consider the part of hyperboloid $\mathcal{L}_{n} \subset \mathbb{R}^{n+1}$ defined as

$$
\mathcal{L}_{n}=\left\{\eta=\left(\tau, \eta_{1}, \ldots, \eta_{n}\right) \in \mathbb{R}^{n+1}, \tau>0, \tau^{2}-\sum \eta_{j}^{2}=1\right\} .
$$

We will consider the point $\overline{0}=(1,0, \ldots, 0)$ as the center of $\mathcal{L}_{n}$.

We will also need a kind of boundary

$$
\bar{B}=\left\{\bar{b}=\left(1, b_{1}, \ldots, b_{n}\right) \in \mathbb{R}^{n+1}, \sum b_{j}^{2}=1\right\}=\left\{\bar{b}=(1, b), b \in \mathbb{S}^{n-1}\right\} .
$$

Notice that $\bar{B}$ does not belong to $\mathcal{L}_{n}$. For any $x, y \in \mathbb{R}^{n+1}$ we set

$$
[x, y]:=x_{1} y_{1}-\sum_{j=2}^{n+1} x_{j} y_{j} .
$$


This bilinear form will be used in three different contexts:

- for $x, y \in \mathcal{L}_{n}$ as an inner product, to define the hyperbolic distance;

- for $x \in \mathcal{L}_{n}, y \in \bar{B}$ as an outer product;

- for $x, y \in \bar{B}$.

The distance $d_{n}(\cdot, \cdot)$ on $\mathcal{L}_{n}$ is defined by the formula

$$
\cosh d_{n}\left(\eta, \eta^{\prime}\right):=\left[\eta, \eta^{\prime}\right]=\tau \tau^{\prime}-\sum \eta_{j} \eta_{j}^{\prime} .
$$

Notice that $[\eta, \eta]=1$, hence $d_{n}(\eta, \eta)=0$. Furthermore,

$$
\left[\eta, \eta^{\prime}\right] \geq \tau \tau^{\prime}-\left(\sum_{j} \eta_{j}^{2}\right)^{1 / 2}\left(\sum_{j}\left(\eta_{j}^{\prime}\right)^{2}\right)^{1 / 2} \geq \tau \tau^{\prime}-\sqrt{\tau^{2}-1} \sqrt{\tau^{\prime 2}-1} \geq 1
$$

hence (4.1) is well defined. We have, in particular,

$$
\cosh d_{n}(\eta, \overline{0})=\tau
$$

and

$$
\sum_{j} \eta_{j}^{2}=\tau^{2}-1=\cosh ^{2} d_{n}(\eta, \overline{0})-1=\sinh ^{2} d_{n}(\eta, \overline{0}) .
$$

Finally, recall what is invariant measure in $\mathcal{L}_{n}$. According to [17], p. 153, the surface measure $A(r)$ of the sphere of radius $r$ is given by

$$
A(r)=c(\sinh r)^{n-1} .
$$

Hence the radial representation of invariant measure is

$$
\mathrm{d} \eta=c(\sinh r)^{n-1} \mathrm{~d} r \mathrm{~d} \theta .
$$

\subsection{Spherical functions on $\mathcal{L}_{\boldsymbol{n}}$}

For any $b \in B=\mathbb{S}^{n-1}$ we write $\bar{b}=(1, b) \in \bar{B}$ and define the family of spherical functions indexed by $s \in \mathbb{C}$, as

$$
\omega_{s}(\eta)=\int_{B}[\eta, \bar{b}]^{-s} \mathrm{~d} b, \quad \eta \in \mathcal{L}_{n} .
$$

Recall that

$$
[\eta, \bar{b}]=\tau-\sum_{j} \eta_{j} b_{j}=\cosh r-\sinh r \cos \theta,
$$

where $r=d_{n}(\eta, \overline{0})$ and $\theta$ the angle between vectors $\eta$ and $b$.

The related Laplace-Beltrami operator (restricted to the class of spherically symmetric functions) can be defined as [11], p. 200,

$$
\Delta f=\frac{\mathrm{d}^{2}}{\mathrm{~d} r^{2}} f+\frac{A^{\prime}(r)}{A(r)} \frac{\mathrm{d}}{\mathrm{d} r} f
$$

where $A$ is defined in (4.3). Since $\frac{A^{\prime}(r)}{A(r)}=(n-1) \operatorname{coth}(r)$, we get

$$
\Delta f=\frac{\mathrm{d}^{2}}{\mathrm{~d} r^{2}} f+(n-1) \operatorname{coth}(r) \frac{\mathrm{d}}{\mathrm{d} r} f .
$$

All spherical functions are eigenfunctions of Laplace-Beltrami operator with eigenvalues $-\lambda_{s}=-s(n-1-s)$ but we are only interested in those corresponding to the negative eigenvalues (i.e. positive $\lambda$ 's). This leads to 
two connected families:

- Upper spectrum: $s=\frac{n-1}{2}+\nu i, \nu \in \mathbb{R}$. The corresponding $\lambda$ 's are $\lambda_{s}=\frac{(n-1)^{2}}{4}+\nu^{2}$ and $\nu,-\nu$ provide the same spherical function. Therefore, it is sufficient to keep $\nu \geq 0$. From the point of view of operators in Hilbert spaces, the Laplace-Beltrami operator is a non-positive symmetric operator on $L^{2}\left(\mathcal{L}_{n}\right)$. Since these spherical functions are in the $L^{2}\left(\mathcal{L}_{n}\right)$, they are eigenfunctions of the Laplace-Beltrami operator in this sense.

- Lower spectrum: $0<s \leq n-1$. The corresponding $\lambda$ 's are $\lambda_{s}=s(n-1-s) \in\left[0, \frac{(n-1)^{2}}{4}\right)$ and again it is sufficient to keep the half-interval $\left[0, \frac{n-1}{2}\right]$ in view of the repetitions.

Notice that $s=\frac{n-1}{2}$ belongs to both spectra.

For $n=2$, we have the upper spectrum $\left(\frac{1}{2}+\nu i, \nu \geq 0\right)$, and the lower spectrum $\left(0, \frac{1}{2}\right]$.

We can give the following more explicit representation for spherical functions. Clearly, $\omega_{s}(\eta)$ depends only on the value of $\sum_{j} \eta_{j}^{2}$. More precisely, let $r=d_{n}(\eta, \overline{0})$. Recall that $\cosh r=[\eta, \overline{0}]=\tau$ and $\sum_{j} \eta_{j}^{2}=\sinh ^{2} r$.

By letting $\theta$ denote the angle between $\left(\eta_{1}, \ldots, \eta_{n}\right)$ and $\left(b_{1}, \ldots, b_{n}\right)$, we get [11], p. 207,

$$
\begin{aligned}
\omega_{s}(\eta) & =\int_{B}\left(\tau-\sum_{j} \eta_{j} b_{j}\right)^{-s} \mathrm{~d} b=\int_{B}(\cosh r-\sinh r \cdot \cos \theta)^{-s} \mathrm{~d} b \\
& =C_{n} \int_{0}^{\pi}(\cosh r-\sinh r \cdot \cos \theta)^{-s} \sin ^{n-2} \theta \mathrm{d} \theta,
\end{aligned}
$$

where

$$
C_{n}=\frac{\Gamma(n / 2)}{\sqrt{\pi} \Gamma((n-1) / 2)}
$$

We will also need a limiting function

$$
Q(\eta)=\lim _{s \rightarrow 0} s^{-1}\left[1-\omega_{s}(\eta)\right]=C_{n} \int_{0}^{\pi} \log (\cosh r-\sinh r \cdot \cos \theta) \sin ^{n-2} \theta \mathrm{d} \theta
$$

called generalized quadratic form. The function $Q$ thus emerges at the point $s=0$ in the spectrum. Notice that $\omega_{0}(\eta) \equiv 1$ and

$$
\Delta Q(\eta)=\lim _{s \rightarrow 0} s^{-1}\left[0-\Delta \omega_{s}(\eta)\right]=\lim _{s \rightarrow 0} s^{-1} s(n-1-s) \omega_{s}(\eta)=(n-1) \omega_{0}(\eta)=n-1 .
$$

For small dimensions one can calculate some of these integrals, e.g. for $n=2$ and $r=d_{n}(\eta, \overline{0})$,

$$
Q(\eta)=2 \log \cosh (r / 2)
$$

and for $n=3$ (the most convenient for calculation)

$$
\begin{gathered}
Q(\eta)=r \operatorname{coth} r-1, \\
\omega_{s}(\eta)=\frac{\sinh ((s-1) r)}{(s-1) \sinh r} .
\end{gathered}
$$

Remark: One can find explicit but more complicated formulas for $Q$ in arbitrary dimension in [28]. The expressions are different for odd and even dimensions.

Remark: When applying the limiting procedure analogous to (4.10) to Euclidean spherical functions from Section 2 , we obtain for any $t \in \mathbb{R}^{n}$

$$
\widehat{Q}(t)=\lim _{s \rightarrow 0} s^{-2}\left[1-\phi_{s}(t)\right]=\frac{1}{2} \int_{\mathbb{S}^{n-1}}(t . \sigma)^{2} \mathrm{~d} \sigma=\frac{\|t\|^{2}}{2 n},
$$


which justifies the name of generalized quadratic form for $Q$. Both $Q$ and $\widehat{Q}$ play the role of degenerate term in respective spectral representations for the fields with stationary increments, see (2.9) above and (5.21) below.

The quadratic form $\widehat{Q}$ also appears as a degenerate term in the Lévy-Khinchin formula for infinite-divisible distributions in $\mathbb{R}^{n}$ where it corresponds to Gaussian distribution. We have no similar role for $Q$ in hyperbolic case.

Notice that all $\omega_{s}(\cdot)$ are bounded functions (they are actually non-negative definite) while $Q$ has a linear growth in $r$, as $r \rightarrow \infty$. We will consider the asymptotic behavior more carefully later.

Now we just mention one useful non-asymptotic inequality. Namely [11], p. 210

$$
\omega_{s}(\eta) \geq 1-Q(\eta) s \geq 1-r s, \quad \forall \eta \in \mathcal{L}_{n}, s \in\left(0, \frac{n-1}{2}\right]
$$

Proof. By (4.4)

$$
\begin{aligned}
\omega_{s}(\eta) & =\int_{B}[\eta, \bar{b}]^{-s} \mathrm{~d} b=\int_{B} \mathrm{e}^{-s \log [\eta, \bar{b}]} \mathrm{d} b \\
& \geq \int_{B}(1-s \log [\eta, \bar{b}]) \mathrm{d} b=1-Q(\eta) s .
\end{aligned}
$$

On the other hand, it is clear from (4.10) that

$$
Q(\eta) \leq \log \cosh (r) \leq r
$$

and we are done.

\subsection{Relation between the disk and hyperboloid models}

\subsubsection{Isometry of Riemannian manifolds}

When $n=2$, we have introduced two models for the hyperbolic plane. We now describe the correspondence between those models. Towards this aim, we recall some elementary facts on Riemannian manifolds, from which we get a complete dictionary. We refer the reader to [16] to fill the gaps in this brief presentation.

In our case a manifold is a surface embedded in some $\mathbb{R}^{m}$. For instance $\mathcal{L}_{2}$ is the surface in $\mathbb{R}^{3}$ defined by $\mathcal{L}_{2}=\left\{\left(\tau, \eta_{1}, \eta_{2}\right) \in \mathbb{R}^{3}, 1=\tau^{2}-\eta_{1}^{2}-\eta_{2}^{2}\right\}$. For every point $\eta=\left(\tau, \eta_{1}, \eta_{2}\right) \in \mathcal{L}_{2}$, one can equip the tangent space at point $\eta$ denoted by $T_{\eta} \mathcal{L}_{2}$ with a scalar product inherited from the Euclidean scalar product in $\mathbb{R}^{3}$. Since $\mathcal{L}_{2}=F^{-1}(1)$ for $F\left(\tau, \eta_{1}, \eta_{2}\right)=\tau^{2}-\eta_{1}^{2}-\eta_{2}^{2}, V=\left(\theta, v_{1}, v_{2}\right) \in T_{\eta} \mathcal{L}_{2}$ if and only if $d F(\eta)(V)=0$ i.e.

$$
\tau \theta=\eta_{1} v_{1}+\eta_{2} v_{2}=(\bar{\eta}, v)_{\mathbb{R}^{2}},
$$

where we denote by $\bar{\eta}=\left(\eta_{1}, \eta_{2}\right)$, by $v=\left(v_{1}, v_{2}\right)$, and by $(\cdot, \cdot)_{\mathbb{R}^{2}}$ the Euclidean scalar product on $\mathbb{R}^{2}$. If $V, V^{\prime}=\left(\theta^{\prime}, v_{1}^{\prime}, v_{2}^{\prime}\right) \in T_{\eta} \mathcal{L}_{2}$ we set by definition

$$
\left(V, V^{\prime}\right)_{T_{\eta} \mathcal{L}_{2}}:=\frac{1}{4}\left(\theta \theta^{\prime}+v_{1} v_{1}^{\prime}+v_{2} v_{2}^{\prime}\right),
$$

which can be rewritten as

$$
\left(V, V^{\prime}\right)_{T_{\eta} \mathcal{L}_{2}}=\frac{1}{4}\left(\left(v, v^{\prime}\right)-\frac{(\bar{\eta}, v)_{\mathbb{R}^{2}}\left(\bar{\eta}, v^{\prime}\right)_{\mathbb{R}^{2}}}{\tau^{2}}\right)
$$

in view of (4.16). We call $\mathcal{L}_{2}$ equipped with this family of scalar products a Riemannian manifold. We can also consider $\mathbb{D}$ as a Riemannian manifold embedded in $\mathbb{R}^{2}$. First, it is an open set in $\mathbb{C}$ which is identified with $\mathbb{R}^{2}$. 
Next, the tangent space $T_{z} \mathbb{D}$ at point $z$, which is identified with $\mathbb{R}^{2}$, is equipped (see [17], p. 29) with the scalar

$$
\left(u_{1}, u_{2}\right)_{T_{z} \mathbb{D}}=\frac{\left(u_{1}, u_{2}\right)_{\mathbb{R}^{2}}}{\left(1-|z|^{2}\right)^{2}} .
$$

Now we introduce a one to one map $\mathcal{I}$ between $\mathcal{L}_{2}$ and $\mathbb{D}$ by associating $\eta$ with $\mathcal{I}(\eta)=z=z_{1}+i z_{2}$ where

$$
z_{j}=\frac{\eta_{j}}{\sqrt{1+\eta_{1}^{2}+\eta_{2}^{2}}+1}
$$

for $j=1,2$. The inverse of $\mathcal{I}$ is then given by

$$
\tau=\frac{1+|z|^{2}}{1-|z|^{2}}, \quad \eta_{j}=\frac{2 z_{j}}{1-|z|^{2}}
$$

for $j=1,2$. Then the inner product transposed to $\mathbb{D}$ is

$$
\left[z, z^{\prime}\right]:=\left[\eta, \eta^{\prime}\right]=\tau \tau^{\prime}-\left(\eta_{1} \eta_{1}^{\prime}+\eta_{2} \eta_{2}^{\prime}\right)=\frac{\left(1+|z|^{2}\right)\left(1+\left|z^{\prime}\right|^{2}\right)-4\left(z_{1} z_{1}^{\prime}+z_{2} z_{2}^{\prime}\right)}{\left(1-|z|^{2}\right)\left(1-\left|z^{\prime}\right|^{2}\right)}
$$

We will now prove that $\mathcal{I}$ is a Riemannian isometry. Namely, we check the equality of the corresponding Riemann structures. We fix $\eta \in \mathcal{L}_{2}$ and $\mathcal{I}(\eta)=z \in \mathbb{D}$ and verify that the scalar product in the tangent space $T_{\eta} \mathcal{L}_{2}$ is mapped to the scalar product of $T_{z} \mathbb{D}$, by the differential of $\mathcal{I}$.

Thus we have to define and compute this differential.

Let $u$ be a tangent vector at point $z \in \mathbb{D}$. Let $z_{\delta}=z+\delta u$ for $\delta$ small enough and $\left(\tau_{\delta}, \eta_{\delta}\right)$ be the corresponding point in $\mathcal{L}_{2}$. By definition,

$$
\tau_{\delta}=\frac{1+\left|z_{\delta}\right|^{2}}{1-\left|z_{\delta}\right|^{2}}
$$

It is easy to see that $\frac{\mathrm{d}\left|z_{\delta}\right|^{2}}{\mathrm{~d} \delta}=2 z \bar{u}$, where we have identified $u \in T_{z} \mathbb{D}$ with the complex number $u=u_{1}+i u_{2}$. Hence,

$$
\theta:=\frac{\mathrm{d} \tau_{\delta}}{\mathrm{d} \delta}=\frac{4 z \bar{u}}{\left(1-|z|^{2}\right)^{2}}
$$

Next, $\eta_{\delta}=\frac{2 z_{\delta}}{1-\left|z_{\delta}\right|^{2}} \cdot$ By differentiating this, we get

$$
v:=\frac{\mathrm{d} \eta_{\delta}}{\mathrm{d} \delta}=\frac{4(z, u)_{\mathbb{R}^{2}}}{z}\left(1-|z|^{2}\right)^{2}+\frac{2 u}{1-|z|^{2}}=\theta z+\frac{2 u}{1-|z|^{2}} .
$$

The relation $u \rightarrow(\theta, v)$ given by the formula above defines the differential $d \mathcal{I}^{-1}$. The differential is easily invertible: we get

$$
\frac{2 u}{1-|z|^{2}}=v-\theta z=v-\frac{(\bar{\eta}, v)_{\mathbb{R}^{2}}}{\tau}
$$

hence,

$$
u=\frac{1-|z|^{2}}{2}\left(v-\frac{(\bar{\eta}, v)_{\mathbb{R}^{2}}}{\tau} z\right),
$$

and we denote the map thus defined $\mathrm{d} \mathcal{I}_{\eta}$. We can now check that if $\mathcal{I}(\eta)=z, V, V^{\prime} \in T_{\eta} \mathcal{L}_{2}$

$$
\left(\mathrm{d} \mathcal{I}_{\eta}(V), \mathrm{d} \mathcal{I}_{\eta}\left(V^{\prime}\right)\right)_{T_{z} \mathbb{D}}=\left(V, V^{\prime}\right)_{T_{\eta} \mathcal{L}_{2}} .
$$

$$
\begin{aligned}
& \text { If } V_{1}=\left(\theta_{1}, v_{1}\right), V_{2}=\left(\theta_{2}, v_{2}\right) \text { and } u_{1}=\mathrm{d}(\mathcal{I})_{\eta} V_{1}, u_{2}=\mathrm{d}(\mathcal{I})_{\eta} V_{2} \\
& \qquad\left(u_{1}, u_{2}\right)_{T_{z} \mathbb{D}}=\frac{\left(u_{1}, u_{2}\right)_{\mathbb{R}^{2}}}{\left(1-|z|^{2}\right)^{2}}=\frac{1}{4}\left(v_{1}-\frac{\left(\bar{\eta}, v_{1}\right)_{\mathbb{R}^{2}}}{\tau} z, v_{2}-\frac{\left(\bar{\eta}, v_{2}\right)_{\mathbb{R}^{2}}}{\tau} z\right)_{\mathbb{R}^{2}}
\end{aligned}
$$


because of (4.23). Hence,

$$
\begin{aligned}
\left(u_{1}, u_{2}\right)_{T_{z} \mathbb{D}} & =\frac{1}{4}\left(v_{1} \frac{\left(\bar{\eta}, v_{1}\right)_{\mathbb{R}^{2}}}{\tau} \frac{\bar{\eta}}{\tau+1}, v_{2} \frac{\left(\bar{\eta}, v_{2}\right)_{\mathbb{R}^{2}}}{\tau} \frac{\bar{\eta}}{\tau+1}\right)_{\mathbb{R}^{2}} \\
& =\frac{1}{4}\left(\left(v_{1}, v_{2}\right)_{\mathbb{R}^{2}}-\frac{\left(\bar{\eta}, v_{1}\right)_{\mathbb{R}^{2}}\left(\bar{\eta}, v_{2}\right)_{\mathbb{R}^{2}}}{\tau^{2}}\left[\frac{2 \tau}{\tau+1}-\frac{\|\bar{\eta}\|_{\mathbb{R}^{2}}^{2}}{(\tau+1)^{2}}\right]\right) \\
& =\frac{1}{4}\left(\left(v_{1}, v_{2}\right)_{\mathbb{R}^{2}}-\frac{\left(\bar{\eta}, v_{1}\right)_{\mathbb{R}^{2}}\left(\bar{\eta}, v_{2}\right)_{\mathbb{R}^{2}}}{\tau^{2}}\left[\frac{2 \tau}{\tau+1}-\frac{\tau^{2}-1}{(\tau+1)^{2}}\right]\right) \\
& =\frac{1}{4}\left(\left(v_{1}, v_{2}\right)_{\mathbb{R}^{2}}-\frac{\left(\bar{\eta}, v_{1}\right)_{\mathbb{R}^{2}}\left(\bar{\eta}, v_{2}\right)_{\mathbb{R}^{2}}}{\tau^{2}}\right) \\
& =\left(V_{1}, V_{2}\right)_{T_{\eta} \mathcal{L}_{2}}
\end{aligned}
$$

and we see that the two scalar products coincide.

\subsubsection{Isometry of metric spaces}

We will now check that $\mathcal{I}$ is an isometry between the metric spaces $\left(\mathcal{L}_{2}, \frac{1}{2} d_{2}\right)$, where $d_{2}$ is defined in (4.1) for $n=2$, and $(\mathbb{D}, d)$, where $d$ is defined in (3.1). Actually it is a straightforward consequence of the facts that $\frac{1}{2} d_{2}$ is the geodesic distance associated with the Riemannian structure (4.17) of $\mathcal{L}_{2}$ and $d$ is the associated geodesic distance with the Riemannian structure (4.19). We briefly recall what is a geodesic distance on a Riemannian manifold. If $\gamma:(-1,1) \mapsto M$ is a $C^{1}$ curve on a Riemannian manifold, the length of $\gamma$ is defined by:

$$
L(\gamma):=\int_{-1}^{1}\left(\frac{\mathrm{d}}{\mathrm{d} t} \gamma(t), \frac{\mathrm{d}}{\mathrm{d} t} \gamma(t)\right)_{T_{\gamma(t)} M}^{\frac{1}{2}} \mathrm{~d} t .
$$

Then the geodesic distance between two points $m, m^{\prime} \in M$ is the minimum of the length of curves such that $\gamma(-1)=m, \gamma(1)=m^{\prime}$. Hence it is obvious that a Riemannian isometry is also an isometry for the associated geodesic distance. See [17], p. 60, for further results. It is classical that the geodesic distance on $\mathbb{D}$ is given by (3.1) (see [17], p. 30) and one can check that $\frac{1}{2} d_{2}$ is the geodesic distance on $\mathcal{L}_{2}$.

Since the just given proof is implicit, some further example would be instructive. In particular, for $z^{\prime}=0$ and any $z \in \mathbb{D}$, we derive from $(4.22)$

$$
[z, 0]=\frac{1+|z|^{2}}{1-|z|^{2}}
$$

which is to be successfully compared to $(3.2)$

$$
\cosh (2 d(z, 0))=\frac{1}{2}\left[\frac{1+|z|}{1-|z|}+\frac{1-|z|}{1+|z|}\right]=\frac{1+|z|^{2}}{1-|z|^{2}} .
$$

We see that $d_{2}(\eta, \overline{0})=2 d(z, 0)$.

\subsubsection{Relation between Laplace operators}

Once a Riemannian structure is defined on a manifold, there is general construction of Laplace-Beltrami operator (see [17], p. 31). Hence, the existence of a simple correspondence between Laplace-Beltrami operator on $\mathcal{L}_{2}$ and on $\mathbb{D}$ is not a surprise. Although it is quite obvious, there is factor two in the correspondence, which we try to make explicit in the following paragraph.

Since the distance in $\mathbb{D}$ is twice shorter than the one in $\mathcal{L}_{2}$, the radial derivative $\frac{\mathrm{d}}{\mathrm{d} r}$ is twice bigger in $\mathbb{D}$. Therefore Helgason's Laplace-Beltrami operator in (3.6) is 4 times bigger that its $\mathcal{L}_{2}$ analogue in (4.6) when $n=2$.

Let us explain this more precisely. For $\eta \in \mathcal{L}_{2}$ we have a corresponding $z \in \mathbb{D}$. For any function $f: \mathcal{L}_{2} \rightarrow \mathbb{R}$ we can define $f_{\mathbb{D}}: \mathbb{D} \rightarrow \mathbb{R}$ by letting $f_{\mathbb{D}}(z)=f(\eta)$. Fix for a while $\eta$ and $z$. Let $\eta^{\prime}$ and $z^{\prime}$ be the corresponding 
points approaching to $\eta$, resp. $z$ along radial geodesic lines from the side opposite to zero. Then the radial derivatives are

$$
\begin{gathered}
\frac{\mathrm{d}_{\mathcal{L}}}{\mathrm{d} r} f(\eta)=\lim _{\eta^{\prime} \rightarrow \eta} \frac{f\left(\eta^{\prime}\right)-f(\eta)}{d_{2}\left(\eta^{\prime}, \eta\right)}, \\
\frac{\mathrm{d}_{\mathbb{D}}}{\mathrm{d} r} f_{\mathbb{D}}(z)=\lim _{z^{\prime} \rightarrow z} \frac{f_{\mathbb{D}}\left(z^{\prime}\right)-f_{\mathbb{D}}(z)}{d\left(z^{\prime}, z\right)} .
\end{gathered}
$$

Since $f\left(\eta^{\prime}\right)-f(\eta)=f_{\mathbb{D}}\left(z^{\prime}\right)-f_{\mathbb{D}}(z)$ and $d_{2}\left(\eta^{\prime}, \eta\right)=2 d\left(z^{\prime}, z\right)$, we have $\frac{\mathrm{d} \mathbb{D}}{\mathrm{d} r} f_{\mathbb{D}}(z)=2 \frac{\mathrm{d} \mathcal{L}}{\mathrm{d} r} f(\eta)$. By iterating this argument, we have $\frac{\mathrm{d}_{\mathrm{D}}^{2}}{\mathrm{~d} r^{2}} f_{\mathbb{D}}(z)=4 \frac{\mathrm{d}_{\mathcal{c}}^{2}}{\mathrm{~d} r^{2}} f(\eta)$. Now comparing Laplace-Beltrami operators in (3.6) and (4.6) is straightforward:

$$
\begin{aligned}
\Delta_{\mathbb{D}} f_{\mathbb{D}}(z) & =\frac{\mathrm{d}_{\mathbb{D}}^{2}}{\mathrm{~d} r^{2}} f_{\mathbb{D}}(z)+2 \operatorname{coth}(2 d(z, 0)) \frac{\mathrm{d}_{\mathbb{D}}}{\mathrm{d} r} f(z) \\
& =4 \frac{\mathrm{d}_{\mathcal{L}}^{2}}{\mathrm{~d} r^{2}} f(\eta)+4 \operatorname{coth}\left(d_{2}(\eta, 0)\right) \frac{\mathrm{d}_{\mathcal{L}}}{\mathrm{d} r} f(\eta) \\
& =4 \Delta_{\mathcal{L}} f(\eta) .
\end{aligned}
$$

\subsubsection{Relation between spherical functions}

Write Helgason expression (3.18) as

$$
\begin{aligned}
\varphi_{\lambda}(z) & =\int_{B} \mathrm{e}^{(\mathrm{i} \lambda+1)\langle z, b\rangle} \mathrm{d} b \\
& =\int_{B}\left[\mathrm{e}^{-2\langle z, b\rangle}\right]^{-(\mathrm{i} \lambda+1) / 2} \mathrm{~d} b \\
& =\int_{B}\left[\frac{|z-b|^{2}}{1-|z|^{2}}\right]^{-(\mathrm{i} \lambda+1) / 2} \mathrm{~d} b .
\end{aligned}
$$

We express now the fraction as a function of $r=d(z, 0)$ and of $\theta$ - the angle between $z$ and $b$, as we did for Faraut spherical functions. Recall that $|z|=\tanh r$, see (3.3) Hence, $1-|z|^{2}=\cosh ^{-2} r$. On the other hand,

$$
|z-b|^{2}=|z|^{2}+1-2|z| \cos \theta=\tanh ^{2} r+1-2 \tanh r \cos \theta
$$

We get

$$
\begin{aligned}
\frac{|z-b|^{2}}{1-|z|^{2}} & =\cosh ^{2} r\left[\tanh ^{2} r+1-2 \tanh r \cos \theta\right] \\
& =\sinh ^{2} r+\cosh ^{2} r-2 \cosh r \sinh r \cos \theta \\
& =\cosh (2 r)-\sinh (2 r) \cos \theta \\
& =[\eta, \bar{b}] .
\end{aligned}
$$

The latter equality follows from (4.5) by using $2 r=2 d(z, 0)=d_{2}(\eta, \overline{0})$. We conclude that Helgason spherical functions reproduce Faraut's spherical functions from upper spectrum. Namely, if $z \in \mathbb{D}$ and $\eta \in \mathcal{L}_{2}$ are chosen as above, so that $d_{2}(\eta, \overline{0})=2 d(z, 0)$, then

$$
\varphi_{\lambda}(z)=\omega_{1 / 2+i \lambda / 2}(\eta) .
$$




\subsection{Asymptotic behavior of spherical functions}

We consider the behavior of functions (4.8) and (4.10) at zero and infinity giving special attention to the case $n=2$. Note that $C_{2}=\frac{\Gamma(1)}{\sqrt{\pi} \Gamma(1 / 2)}=\pi^{-1}$ and we get from (4.8) and (4.10) for $n=2$

$$
\begin{aligned}
& \omega_{s}(\eta)=\pi^{-1} \int_{0}^{\pi}(\cosh r-\sinh r \cdot \cos \theta)^{-s} \mathrm{~d} \theta \\
& Q(\eta)=\pi^{-1} \int_{0}^{\pi} \log (\cosh r-\sinh r \cdot \cos \theta) \mathrm{d} \theta .
\end{aligned}
$$

\subsubsection{Behavior at infinity}

The study of the asymptotic behavior of spherical functions is again a classical topic in harmonic analysis. Since it uses tools familiar to probabilists, we briefly recall results.

Proposition 4.1. For any $0<s<\frac{n-1}{2}$, it is true that

$$
\omega_{s}(\eta)=C(s, n) \mathrm{e}^{-s r}\left(1+O\left(\mathrm{e}^{-2 r}\right)+O\left(\mathrm{e}^{-2 r\left(\frac{n-1}{2}-s\right)}\right)\right), \quad r \rightarrow \infty,
$$

with

$$
C(s, n)=C_{n} 2^{s} \int_{0}^{\pi}(1-\cos \theta)^{-s} \sin ^{n-2} \theta \mathrm{d} \theta .
$$

For $s=\frac{n-1}{2}$ which is the boundary between upper and lower spectrum we have

$$
\omega_{s}(\eta)=C\left(\frac{n-1}{2}, n\right) \mathrm{e}^{-\frac{(n-1) r}{2}}(r+O(1)), \quad r \rightarrow \infty .
$$

For the upper spectrum we have the same uniform bound, since

$$
\left|\omega_{\frac{n-1}{2}+\mathrm{i} \lambda}(\eta)\right| \leq \omega_{\frac{n-1}{2}}(\eta)
$$

which can be used along with (4.29).

Proof. Since for fixed $\theta$

$$
\begin{gathered}
\cosh r-\sinh r \cdot \cos \theta=\frac{\mathrm{e}^{r}}{2}\left(1-\cos \theta+\mathrm{e}^{-2 r}(1+\cos \theta)\right), \\
\omega_{s}(\eta)=C_{n} \mathrm{e}^{-s r} 2^{s} \int_{0}^{\pi}\left(1-\cos \theta+\mathrm{e}^{-2 r}(1+\cos \theta)\right)^{-s} \sin ^{n-2} \theta \mathrm{d} \theta \\
=C_{n} \mathrm{e}^{-s r} 2^{s} \int_{0}^{\pi}(1-\cos \theta)^{-s} \sin ^{n-2} \theta \mathrm{d} \theta\left(1+O\left(\mathrm{e}^{-2 r}\right)+O\left(\mathrm{e}^{-2 r\left(\frac{n-1}{2}-s\right)}\right)\right) .
\end{gathered}
$$

Note that the integral converges exactly for $s<\frac{n-1}{2}$. We have thus (4.27).

By using (4.9) and a formula from [9], p. 361,

$$
\int_{0}^{\pi}(1-\cos \theta)^{-s} \sin ^{n-2} \theta \mathrm{d} \theta=2^{n-2-s} \frac{\Gamma\left(\frac{n-1}{2}-s\right) \Gamma\left(\frac{n-1}{2}\right)}{\Gamma(n-1-s)},
$$

we obtain

$$
C(s, n)=2^{n-2} \frac{\Gamma\left(\frac{n}{2}\right) \Gamma\left(\frac{n-1}{2}-s\right)}{\sqrt{\pi} \Gamma(n-1-s)}, \quad 0<s<\frac{n-1}{2} .
$$


In particular case $n=2$,

$$
C(s, 2)=\frac{\Gamma\left(\frac{1}{2}-s\right)}{\sqrt{\pi} \Gamma(1-s)}, \quad 0<s<\frac{1}{2} .
$$

We come to critical case $s=\frac{n-1}{2}$. Just split the integral in two parts $\left.\left(0, \mathrm{e}^{-r}\right)\right]$ and $\left(\mathrm{e}^{-r}, \pi\right]$ and obtain (4.29). To obtain (4.30), it is enough to write

$$
\left|\omega_{\frac{n-1}{2}+\mathrm{i} \lambda}(\eta)\right| \leq \int_{B}\left|[\eta, \bar{b}]^{-s}\right| \mathrm{d} b=\int_{B}[\eta, \bar{b}]^{-\frac{n-1}{2}} \mathrm{~d} b=\omega_{\frac{n-1}{2}}(\eta) .
$$

In Theorem 4.5 of [16] the following asymptotic behavior is proved for $n=2$ in terms of the disk model:

$$
\varphi_{\lambda}(z) \sim \mathrm{e}^{-r} \mathrm{e}^{\mathrm{i} \lambda r} c(\lambda), \quad r=d(z, 0) \rightarrow \infty, \quad \text { if } \Re(\mathrm{i} \lambda)>0,
$$

where

$$
c(\lambda)=\frac{\Gamma(\mathrm{i} \lambda / 2)}{\pi^{1 / 2} \Gamma((\mathrm{i} \lambda+1) / 2)} .
$$

is Harish-Chandra function. Notice that standard formulas $|\Gamma(\mathrm{i} y)|^{2}=\frac{\pi}{y \sinh (\pi y)}$ and $|\Gamma(1 / 2+\mathrm{i} y)|^{2}=\frac{\pi}{\cosh (\pi y)}$ yield

$$
|c(\lambda)|^{-2}=\left[\frac{2 \cosh (\pi \lambda / 2)}{\pi \lambda \sinh (\pi \lambda / 2)}\right]^{-1}=\frac{\pi \lambda \tanh (\pi \lambda / 2)}{2}
$$

which somehow explains the tanh in Fourier transform.

By applying (4.32) to $\lambda=(2 s-1)$ i, and moving to hyperboloid model, we see that (4.32) yields exactly the same asymptotics as (4.27) with the constant (4.31).

Now we move to the behavior of the generalized quadratic form at infinity.

Proposition 4.2. Let us define $q_{n}$ by the formula

$$
q_{n}(\eta):=Q(\eta)-r+\tilde{C}_{n}
$$

where $\tilde{C}_{n}=\log 2-C_{n} \int_{0}^{\pi} \log (1-\cos \theta) \sin ^{n-2} \theta \mathrm{d} \theta$.

Then, as $r=d_{n}(\eta, 0) \rightarrow \infty$,

$$
q_{n}(\eta)= \begin{cases}O\left(\mathrm{e}^{-r}\right) & n=2 \\ O\left(r \mathrm{e}^{-2 r}\right) & n=3 \\ O\left(\mathrm{e}^{-2 r}\right) & n>3\end{cases}
$$

Proof. One can write

$$
\begin{aligned}
Q(\eta)= & r-\log 2+C_{n} \int_{0}^{\pi} \log \left(1+\mathrm{e}^{-2 r}-\left(1-\mathrm{e}^{-2 r}\right) \cos \theta\right) \sin ^{n-2} \theta \mathrm{d} \theta \\
= & r-\log 2+C_{n} \int_{0}^{\pi} \log (1-\cos \theta) \sin ^{n-2} \theta \mathrm{d} \theta \\
& +C_{n} \int_{0}^{\pi} \log \left(1+\frac{\mathrm{e}^{-2 r}(1+\cos \theta)}{1-\cos \theta}\right) \sin ^{n-2} \theta \mathrm{d} \theta \\
= & r-\tilde{C}_{n}+q_{n}(\eta) .
\end{aligned}
$$


To see (4.35), for $n=2$ and $n=3$ the easiest way is to look at explicit formulas (4.12) and (4.13). For $n>3$ we simply use the elementary bound

$$
1-\cos \theta=2 \sin ^{2}(\theta / 2) \geq 2\left(\frac{\theta / 2}{\pi / 2}\right)^{2}=\frac{2 \theta^{2}}{\pi^{2}}, \quad 0 \leq \theta \leq \pi
$$

and have

$$
q_{n}(\eta) \leq \mathrm{e}^{-2 r} C_{n} \int_{0}^{\pi} \frac{2}{1-\cos \theta} \theta^{n-2} \mathrm{~d} \theta \leq \mathrm{e}^{-2 r} C_{n} \pi^{2} \int_{0}^{\pi} \theta^{n-4} \mathrm{~d} \theta:=c \mathrm{e}^{-2 r}
$$

Remark: For $n=2$, we find in the tables of integrals

$$
\int_{0}^{\pi} \log (1-\cos \theta) \mathrm{d} \theta=\int_{0}^{\pi / 2} \log (1-\cos \theta) \mathrm{d} \theta+\int_{0}^{\pi / 2} \log (1+\cos \theta) \mathrm{d} \theta=-\pi \log 2
$$

Hence,

$$
Q(\eta)=r-2 \log 2+O\left(\mathrm{e}^{-r}\right), \quad r \rightarrow \infty
$$

in accordance with (4.12).

\subsubsection{Behavior at zero}

When $r \rightarrow 0$, we have

$$
\cosh r-\sinh r \cdot \cos \theta=1+\frac{r^{2}}{2}-r \cos \theta+O\left(r^{3}\right)
$$

Hence,

$$
\begin{aligned}
\log (\cosh r-\sinh r \cdot \cos \theta) & =\frac{r^{2}}{2}-r \cos \theta-\frac{r^{2} \cos ^{2} \theta}{2}+O\left(r^{3}\right) \\
& =-r \cos \theta+\frac{r^{2} \sin ^{2} \theta}{2}+O\left(r^{3}\right) .
\end{aligned}
$$

By integrating we get

$$
\begin{aligned}
Q(\eta) & =C_{n} \int_{0}^{\pi}\left(-r \cos \theta+\frac{r^{2} \sin ^{2} \theta}{2}\right) \sin ^{n-2} \theta \mathrm{d} \theta+O\left(r^{3}\right) \\
& =C_{n} r^{2} \int_{0}^{\pi} \frac{\sin ^{n} \theta}{2} \mathrm{~d} \theta+O\left(r^{3}\right) \\
& =\frac{C_{n}}{2 C_{n+2}} r^{2}+O\left(r^{3}\right)=\frac{(n-1) r^{2}}{2 n}+O\left(r^{3}\right), \quad r \rightarrow 0 .
\end{aligned}
$$


Similarly

$$
\begin{aligned}
\omega_{s}(\eta)-1= & C_{n} \int_{0}^{\pi}(\exp \{-s \log (\cosh r-\sinh r \cdot \cos \theta)\}-1) \mathrm{d} \theta \\
= & -C_{n} s \int_{0}^{\pi} \log (\cosh r-\sinh r \cdot \cos \theta) \sin ^{n-2} \theta \mathrm{d} \theta \\
& +C_{n} \frac{s^{2}}{2} \int_{0}^{\pi} \log ^{2}(\cosh r-\sinh r \cdot \cos \theta) \sin ^{n-2} \theta \mathrm{d} \theta+O\left(r^{3}\right) \\
= & \frac{-s(n-1) r^{2}}{2 n}+C_{n} \frac{s^{2} r^{2}}{2} \int_{0}^{\pi} \cos ^{2} \theta \sin ^{n-2} \theta \mathrm{d} \theta+O\left(r^{3}\right) \\
= & \frac{-s(n-1) r^{2}}{2 n}+\frac{s^{2} r^{2}}{2}\left(1-\frac{C_{n}}{C_{n+2}}\right)+O\left(r^{3}\right) \\
= & \frac{-s(n-1) r^{2}}{2 n}+\frac{s^{2} r^{2}}{2}\left(1-\frac{n-1}{n}\right)+O\left(r^{3}\right) \\
= & \frac{-\left((n-1) s-s^{2}\right)}{2 n} r^{2}+O\left(r^{3}\right), \quad r \rightarrow 0 .
\end{aligned}
$$

For example, $n=3$ yields $\omega_{s}(\eta)=1-\frac{2 s-s^{2}}{6} r^{2}+O\left(r^{3}\right)$, in accordance with (4.14).

Remark: For more detailed and general information about the behavior of spherical functions see [35].

\section{Homogeneous Fields}

In this section we consider the following framework. Let $G$ be a group acting transitively on a space $T$ with a marked point $o$. Let $G_{0}=\{\rho \in G: \rho o=o\}$. Then we can identify $T$ with $G / G_{0}$.

We say that the action of $G$ is rotationally transitive if there is a $G$-invariant metrics $d(\cdot, \cdot)$ on $T$ and for any $t_{1}, t_{2} \in T$ such that $d\left(t_{1}, o\right)=d\left(t_{2}, o\right)$ there exists $\rho \in G_{0}$ such that $\rho t_{1}=t_{2}$.

\subsection{Stationary fields}

Definition 5.1. We say that a complex-valued Gaussian random field $\left\{X_{t}, t \in T\right\}$ is a stationary field if $\forall t_{1}, t_{2} \in T, g \in G$ it is true that $\mathbb{E} X_{t_{1}}=\mathbb{E} X_{t_{2}}$ and $\operatorname{cov}\left(X_{g t_{1}}, X_{g t_{2}}\right)=\operatorname{cov}\left(X_{t_{1}}, X_{t_{2}}\right)$. In the following we assume that $\mathbb{E} X_{t}=0$ for all $t \in T$.

One can extend the definition 2.2 of non-negative definite functions to the framework of this section.

Definition 5.2. A real valued function $f$ on $T$ is called non-negative definite if for every $d \geq 1, \lambda_{1}, \ldots, \lambda_{d} \in \mathbb{C}$, $t_{1}, \ldots, t_{d} \in T$

$$
\sum_{i, j=1}^{d} \lambda_{i} \overline{\lambda_{j}} f\left(t_{i}, t_{j}\right) \geq 0 .
$$

Similarly one can define non-negative definite functions on $G$.

Definition 5.3. A real valued function $f$ on $G$ is called non-negative definite if $F: G \times G \mapsto \mathbb{C}$ defined by $F\left(g_{1}, g_{2}\right)=f\left(g_{2}^{-1} g_{1}\right)$ is non-negative definite on $G$ as in the sense of Definition 5.2.

Covariance function of $X$ is defined as $K: T \times T \rightarrow \mathbb{C}$ by $K(t)=\operatorname{cov}\left(X_{t}, X_{o}\right)$. We can also define its version $\tilde{K}: G \rightarrow \mathbb{C}$ by $\tilde{K}(g)=K(g o)$. Then $\tilde{K}$ is a non-negative definite function, since

$$
\sum_{i, j} c_{i} \bar{c}_{j} \tilde{K}\left(g_{j}^{-1} g_{i}\right)=\mathbb{E}\left|\sum_{j} c_{j} X_{g_{j}}\right|^{2} \geq 0 .
$$


Notice also that $\tilde{K}$ is $G_{0}$-bi-invariant since for any $g \in G, \rho \in G_{0}$

$$
\begin{gathered}
\tilde{K}(g \rho)=K(g \rho o)=K(g o)=\tilde{K}(g), \\
\tilde{K}(\rho g)=K(\rho g o)=\operatorname{cov}\left(X_{\rho g o}, X_{\rho o}\right)=\operatorname{cov}\left(X_{g o}, X_{o}\right)=\tilde{K}(g) .
\end{gathered}
$$

We always have the symmetry

$$
\tilde{K}\left(g^{-1}\right)=\operatorname{cov}\left(X_{g^{-1} o}, X_{o}\right)=\operatorname{cov}\left(X_{o}, X_{g o}\right)=\overline{\operatorname{cov}\left(X_{g o}, X_{o}\right)}=\overline{\tilde{K}(g)} .
$$

Moreover, if the action of $G$ is rotationally transitive, we have $d\left(g^{-1} o, o\right)=d(o, g o)$, hence there exists $\rho \in G_{0}$ such that $g^{-1} o=\rho g o$ and, by $G_{0}$-invariance,

$$
\tilde{K}\left(g^{-1}\right)=\tilde{K}(g) .
$$

By comparing this with general symmetry, we conclude that in this case covariance is always real.

Rather strangely, the covariance function $\tilde{K}$ depends on the choice of the marked point. Indeed, if we take any $g^{\prime} \in G$ and pass from $o$ to $o^{\prime}:=g^{\prime} o$, we get for any $g \in G$,

$$
\begin{aligned}
\tilde{K}^{\prime}(g) & :=\operatorname{cov}\left(X_{g o^{\prime}}, X_{o^{\prime}}\right)=\operatorname{cov}\left(X_{g g^{\prime} o}, X_{g^{\prime} o}\right) \\
& =\operatorname{cov}\left(X_{\left(g^{\prime}\right)^{-1} g g^{\prime} o}, X_{o}\right)=\tilde{K}\left(\left(g^{\prime}\right)^{-1} g g^{\prime}\right) .
\end{aligned}
$$

We see that $\tilde{K}^{\prime}=\tilde{K}$ if $G$ is a commutative group (in particular, for the classical setting of translations in Euclidean space).

In the sequel, we apply this to the case $G=S U(1,1), G_{0}$ subgroup of rotations, $G / G_{0}=\mathbb{D}$.

Bochner theorems enable to select the "basic" covariances and represent arbitrary covariance function as a mixture of the primary ones. For the case $T=\mathcal{L}_{n}, G$ being the group of linear transformations of $\mathbb{R}^{n+1}$ preserving the quadratic form $[\cdot, \cdot]$ (and actually for a much more general case) the following Godement's theorem accomplishes this task by using the spherical functions as the primary ones [11], p. 182, [14]. Recall that the set of non-negatively definite spherical functions is $\left\{\omega_{s}, s \in \mathcal{S}\right\}$, where

$$
\mathcal{S}=\left(0, \frac{n-1}{2}\right) \cup\left\{\frac{n-1}{2}+\mathrm{i} \lambda, \lambda \geq 0\right\}
$$

Theorem 5.4. Let $K: \mathcal{L}_{n} \rightarrow \mathbb{C}$ be a covariance function of a stochastically continuous stationary field. Then there exists a unique finite measure $\nu$ on $\mathcal{S}$ such that

$$
K(\eta)=\int_{\mathcal{S}} \omega_{s}(\eta) \nu(\mathrm{d} s), \quad \eta \in \mathcal{L}_{n}
$$

Conversely, any function of type (5.1) is a covariance function of a stationary field.

The measure $\nu$ is called spectral measure for any field with covariance $K$.

Remark: When we compare Bochner Theorem for hyperbolic space and (2.2) on Euclidean space, there is a minor difference. In (2.2), the spectral measure is an arbitrary measure on $\mathbb{R}^{n}$, but if we let $G$ be the group of all isometries on $\mathbb{R}^{n}$, and not only translations, then stationary Gaussian fields in the sense of Definition 5.1 are stationary as in Definition 2.1 and isotropic because $\left(X_{\rho t}\right)_{t \in \mathbb{R}^{n}} \stackrel{(d)}{=}\left(X_{t}\right)_{t \in \mathbb{R}^{n}}$ for every rotation $\rho$. Hence starting from (2.2) and using a polar decomposition of $\mu$, which is rotationally invariant as $\nu(\mathrm{d} s) \times \sigma(\mathrm{d} \xi)$, where $\sigma(\mathrm{d} \xi)$ 
is the Lebesgue surface measure on the sphere $\mathbb{S}^{n-1}$, we get

$$
\begin{aligned}
r(t) & =\int_{\mathbb{R}^{n}} \mathrm{e}^{\mathrm{i} t \cdot \xi} \mu(\mathrm{d} \xi) \\
& =\int_{0}^{+\infty} \omega_{s}(\|t\|) \nu(\mathrm{d} s),
\end{aligned}
$$

where $\omega_{s}(\|t\|)=\int_{\mathbb{S}^{n-1}} \mathrm{e}^{\mathrm{i} t \cdot \xi} \mathrm{d} \sigma(\xi)$. Note that in the Euclidean case $\omega_{s}$ are Bessel functions.

\subsection{Stationary fields related to upper spectrum}

The basic examples of stationary fields are those with degenerated spectral measure concentrated in one point. It means that the covariance of such field coincides with one of spherical functions. We start with $n=2$ and upper spectrum of hyperbolic space $\mathbb{D}$ as suggested by Baudoin.

Let $W$ be a white noise defined on the boundary circle $B$. Fix $\lambda \in \mathbb{R}$ and define a field $\left\{X_{z}^{\lambda}, z \in \mathbb{D}\right\}$ by

$$
X_{z}^{\lambda}:=\int_{B} \mathrm{e}^{(\mathrm{i} \lambda+1)\langle z, b\rangle} W(\mathrm{~d} b)
$$

Let us first prove that $X^{\lambda}$ is stationary. Indeed, take any $h_{1}, h_{2}, g \in S U(1,1)$, set $z_{1}=h_{1}(0), z_{2}=h_{2}(0)$ and write, by using Lemma 3.5

$$
\begin{aligned}
\operatorname{cov}\left(X_{g z_{1}}^{\lambda}, X_{g z_{2}}^{\lambda}\right) & =\int_{B} \mathrm{e}^{(\mathrm{i} \lambda+1)\left\langle g h_{1}(0), b\right\rangle} \overline{\mathrm{e}^{(\mathrm{i} \lambda+1)\left\langle g h_{2}(0), b\right\rangle}} \mathrm{d} b \\
& =\int_{B} \mathrm{e}^{(\mathrm{i} \lambda+1)\left\langle\left(g h_{2}\right)^{-1} g h_{1}(0), b\right\rangle} \mathrm{d} b \\
& =\int_{B} \mathrm{e}^{(\mathrm{i} \lambda+1)\left\langle h_{2}^{-1} h_{1}(0), b\right\rangle} \mathrm{d} b \\
& =\operatorname{cov}\left(X_{z_{1}}^{\lambda}, X_{z_{2}}^{\lambda}\right) .
\end{aligned}
$$

By specifying here $z_{2}=0$ we find the covariance function

$$
K^{\lambda}(z)=\operatorname{cov}\left(X_{z}^{\lambda}, X_{0}^{\lambda}\right)=\int_{B} \mathrm{e}^{(\mathrm{i} \lambda+1)\langle z, b\rangle} \mathrm{d} b=\varphi_{\lambda}(z)
$$

Moreover, we can mix the above fields as we wish. Take a finite measure $\nu$ on $\mathbb{R}^{+}$, take a white noise $W$ on $\mathbb{R}^{+} \times B$ controlled by the measure $\nu(\mathrm{d} \lambda) \mathrm{d} b$ and construct a process

$$
X_{z}^{\nu}:=\int_{0}^{\infty} \int_{B} \mathrm{e}^{(\mathrm{i} \lambda+1)\langle z, b\rangle} W(\mathrm{~d} b \mathrm{~d} \lambda) .
$$

It is clear that $X^{\nu}$ is a stationary field with covariance

$$
K^{\nu}(z)=\operatorname{cov}\left(X_{z}^{\nu}, X_{0}^{\nu}\right)=\int_{0}^{\infty} \varphi_{\lambda}(z) \nu(\mathrm{d} \lambda) .
$$

We will see a construction of $X^{\nu}$, when $\nu$ admits a density in Section 6.2. Now we move to arbitrary $n$ where we use hyperboloid model. The analytic background of this part (and the next one) follows [9], p. 359-362. Take $s \in(0, n-1] \cup\left(\frac{n-1}{2}+i \mathbb{R}\right)$. Recall that this set parameterizes the spectrum of Laplace-Beltrami operator 
and the points $s$ and $n-1-s$ correspond to the same spherical function $\omega_{s}$. Recall that the corresponding covariance is defined as follows: if $\eta_{2}=g \eta_{1}$, then $K_{s}\left(\eta_{1}, \eta_{2}\right)=\omega_{s}(g \overline{0})$. Define Poisson kernel

$$
P_{s}(\eta, \bar{b})=[\eta, \bar{b}]^{-s}, \quad \eta \in \mathcal{L}_{n}, \bar{b} \in \bar{B} .
$$

According to [9], the covariance associated to spherical function $\omega_{s}$ given in (4.4) admits the following representation

$$
K_{s}\left(\eta_{1}, \eta_{2}\right)=\int_{\bar{B}} P_{s}\left(\eta_{1}, \bar{b}\right) P_{n-1-s}\left(\eta_{2}, \bar{b}\right) \mathrm{d} \bar{b}=\int_{\bar{B}}\left[\eta_{1}, \bar{b}\right]^{-s}\left[\eta_{2}, \bar{b}\right]^{-(n-1-s)} \mathrm{d} \bar{b}
$$

For the upper spectrum, we have $s=(n-1+\mathrm{i} \lambda) / 2, n-1-s=(n-1-i \lambda) / 2$ and we get

$$
\begin{aligned}
K_{s}\left(\eta_{1}, \eta_{2}\right) & =\int_{\bar{B}}\left[\eta_{1}, \bar{b}\right]^{-(n-1+\mathrm{i} \lambda) / 2}\left[\eta_{2}, \bar{b}\right]^{-((n-1-\mathrm{i} \lambda) / 2)} \mathrm{d} \bar{b} \\
& =\int_{\bar{B}}\left[\eta_{1}, \bar{b}\right]^{-(n-1+\mathrm{i} \lambda) / 2} \overline{\left[\eta_{2}, \bar{b}\right]^{-((n-1+\mathrm{i} \lambda) / 2)}} \mathrm{d} \bar{b} .
\end{aligned}
$$

We have thus an integral representation

$$
X^{\lambda}(\eta):=\int_{\bar{B}}[\eta, \bar{b}]^{-(n-1+\mathrm{i} \lambda) / 2} W(\mathrm{~d} \bar{b}) .
$$

Recall that for $n=2$ this expression reduces to (5.2) due to (4.25).

\subsection{Stationary fields related to the lower spectrum}

\subsubsection{Covariance representation}

Now we pass to the basic fields related to the spherical functions of lower spectrum. Take $s \in(0, n-1]$. Recall that this interval parameterizes the lower spectrum and the points $s$ and $n-1-s$ correspond to the same spherical function $\omega_{s}$. Recall that the corresponding covariance is defined as follows: if $\eta_{2}=g \eta_{1}$, then $K_{s}\left(\eta_{1}, \eta_{2}\right)=\omega_{s}(g(\overline{0}))$.

We use Poisson kernel (5.5). There is a formula in [9] that links $P_{s}$ and $P_{n-1-s}$ : for $0<s<\frac{n-1}{2}$,

$$
P_{s}\left(\eta_{1}, \bar{b}\right)=\gamma^{-1}(s, n) \int_{\bar{B}} P_{n-1-s}\left(\eta_{1}, \bar{b}_{1}\right)\left[\bar{b}_{1}, \bar{b}\right]^{-s} \mathrm{~d} \bar{b}_{1}=\gamma^{-1}(s, n) \int_{\bar{B}}\left[\eta_{1}, \bar{b}\right]^{-(n-1-s)}\left[\bar{b}_{1}, \bar{b}\right]^{-s} \mathrm{~d} \bar{b}_{1} .
$$

Here

$$
\gamma(s, n)=2^{n-1-s} \frac{\Gamma(n / 2) \Gamma\left(\frac{n-1}{2}-s\right)}{\sqrt{\pi} \Gamma(n-1-s)} .
$$

By combining this formula with (5.6) we get

$$
K_{s}\left(\eta_{1}, \eta_{2}\right)=\gamma^{-1}(s, n) \int_{\bar{B}} \int_{\bar{B}}\left[\eta_{1}, \bar{b}_{1}\right]^{-(n-1-s)}\left[\eta_{2}, \bar{b}_{2}\right]^{-(n-1-s)}\left[\bar{b}_{1}, \bar{b}_{2}\right]^{-s} \mathrm{~d} \bar{b}_{1} \mathrm{~d} \bar{b}_{2} .
$$

Since by definition

$$
\left[\bar{b}_{1}, \bar{b}_{2}\right]=1-\sum_{j=1}^{n}\left(\bar{b}_{1}\right)_{j}\left(\bar{b}_{2}\right)_{j}=\frac{1}{2}\left\|\bar{b}_{1}-\bar{b}_{2}\right\|^{2},
$$

we get

$$
K_{s}\left(\eta_{1}, \eta_{2}\right)=(2 \gamma(s, n))^{-1} \int_{\bar{B}} \int_{\bar{B}}\left[\eta_{1}, \bar{b}_{1}\right]^{-(n-1-s)}\left[\eta_{2}, \bar{b}_{2}\right]^{-(n-1-s)}\left\|\mid \bar{b}_{1}-\bar{b}_{2}\right\|^{-2 s} \mathrm{~d} \bar{b}_{1} \mathrm{~d} \bar{b}_{2}
$$




\subsubsection{Series representation}

From (5.8) we can deduce a series representation for $X$, at least for $n=2$. Let

$$
a_{m, s}=\int_{0}^{2 \pi} \mathrm{e}^{-\mathrm{i} m \theta}\left\|\mathrm{e}^{\mathrm{i} \theta}-1\right\|^{-2 s} \mathrm{~d} \theta, \quad 0<s<1 / 2 .
$$

Consider a bilinear form

$$
I_{s}\left(f_{1}, f_{2}\right)=\int_{0}^{2 \pi} \int_{0}^{2 \pi} f_{1}\left(\mathrm{e}^{\mathrm{i} \theta_{1}}\right) \overline{f_{2}\left(\mathrm{e}^{\mathrm{i} \theta_{2}}\right)}\left\|\mathrm{e}^{\mathrm{i} \theta_{1}}-\mathrm{e}^{\mathrm{i} \theta_{2}}\right\|^{-2 s} \mathrm{~d} \theta_{1} \mathrm{~d} \theta_{2} .
$$

For $f_{1}=\mathrm{e}^{\mathrm{i} m_{1} \theta}, f_{2}=\mathrm{e}^{\mathrm{i} m_{2} \theta}$ we have

$$
\begin{aligned}
I_{s}\left(f_{1}, f_{2}\right) & =\int_{0}^{2 \pi} \int_{0}^{2 \pi} \mathrm{e}^{\mathrm{i} m_{1} \theta_{1}-\mathrm{i} m_{2} \theta_{2}}\left\|\mathrm{e}^{\mathrm{i} \theta_{1}}-\mathrm{e}^{\mathrm{i} \theta_{2}}\right\|^{-2 s} \mathrm{~d} \theta_{1} \mathrm{~d} \theta_{2} \\
& =\int_{0}^{2 \pi} \mathrm{e}^{\mathrm{i}\left(m_{1}-m_{2}\right) \theta_{1}} \int_{0}^{2 \pi} \mathrm{e}^{-\mathrm{i} m_{2}\left(\theta_{2}-\theta_{1}\right)}\left\|\mathrm{e}^{\mathrm{i} \theta_{1}}-\mathrm{e}^{\mathrm{i} \theta_{2}}\right\|^{-2 s} \mathrm{~d} \theta_{2} \mathrm{~d} \theta_{1} \\
& =\int_{0}^{2 \pi} \mathrm{e}^{\mathrm{i}\left(m_{1}-m_{2}\right) \theta_{1}} a_{m, s} \mathrm{~d} \theta_{1}=2 \pi a_{m, s} \mathbf{1}_{\left\{m_{1}=m_{2}\right\}} .
\end{aligned}
$$

Hence, for all trigonometric polynomials $f_{1}=\sum_{m} \hat{f}_{1 m} \mathrm{e}^{\mathrm{i} m \theta}, f_{2}=\sum_{m} \hat{f}_{2 m} \mathrm{e}^{\mathrm{i} m \theta}$ we have

$$
I_{s}\left(f_{1}, f_{2}\right)=2 \pi \sum_{m \in \mathbb{Z}} a_{m, s} \hat{f}_{1 m} \overline{\hat{f}_{1 m}}
$$

The latter formula remains true for all sufficiently smooth functions $f_{1}, f_{2}$. By taking $\eta_{1}, \eta_{2} \in \mathcal{L}_{2}$ and considering for $j=1,2$ the smooth functions $f_{j}=\left[\eta_{j}, \mathrm{e}^{\mathrm{i} \theta}\right]^{-(1-s)}$ we obtain from (5.7)

$$
K_{s}\left(\eta_{1}, \eta_{2}\right)=\pi \gamma(s, 2)^{-1} \sum_{m \in \mathbb{Z}} a_{m, s} \hat{f}_{1 m} \overline{\hat{f}_{2 m}}
$$

One can easily obtain a field with such covariance by letting

$$
X_{\eta}=\pi^{1 / 2} \gamma(s, 2)^{-1 / 2} \sum_{m \in \mathbb{Z}} a_{m, s}^{1 / 2} \hat{f}_{m}(\eta) \xi_{m}
$$

where $\xi_{m}$ are non-correlated variables and

$$
\hat{f}_{m}(\eta)=\frac{1}{2 \pi} \int_{0}^{2 \pi} \mathrm{e}^{-\mathrm{i} m \theta}\left[\eta, \mathrm{e}^{\mathrm{i} \theta}\right]^{-(1-s)} \mathrm{d} \theta .
$$

\subsubsection{Integral representation}

In this subsection, we give an integral representation of a process $X^{s}$ with covariance (5.8).

Following [23], Section 4.4, we introduce a stereographic projection, which is the inverse of the following map

$$
\mathcal{P}: \mathbb{R}^{n-1} \rightarrow \mathbb{S}^{n-1} \subset \mathbb{R}^{n}
$$

defined by

$$
s_{j}=\frac{2 x_{j}}{1+\|x\|^{2}}, \quad 1 \leq j \leq n-1, \quad s_{n}=\frac{1-\|x\|^{2}}{1+\|x\|^{2}}
$$


The Jacobian of $\mathcal{P}$ is

$$
J_{\mathcal{P}}(x)=\left(\frac{2}{1+\|x\|^{2}}\right)^{n-1}
$$

Let $s \in\left(0, \frac{n-1}{2}\right)$. Then $2-\frac{2 s}{n-1} \in(1,2)$ and $q:=2\left(2-\frac{2 s}{n-1}\right)^{-1} \in(1,2)$. Define the stereographic projection of functions

$$
\mathcal{P}_{q}: L^{q}\left(\mathbb{S}^{n-1}\right) \rightarrow L^{q}\left(\mathbb{R}^{n-1}\right)
$$

by

$$
\left[\mathcal{P}_{q} F\right](x)=J_{\mathcal{P}}(x)^{1 / q} F(\mathcal{P}(x)) .
$$

Then $\left\|\mathcal{P}_{q} F\right\|_{q}=\|F\|_{q}$ but, most importantly, as shown in [23], Section 4.5,

$$
\int_{\mathbb{S}^{n-1}} \int_{\mathbb{S}^{n-1}} F_{1}\left(b_{1}\right) F_{2}\left(b_{2}\right)\left\|b_{1}-b_{2}\right\|^{-2 s} \mathrm{~d} b_{1} \mathrm{~d} b_{2}=\int_{\mathbb{R}^{n-1}} \int_{\mathbb{R}^{n-1}}\left[\mathcal{P}_{q} F_{1}\right]\left(x_{1}\right)\left[\mathcal{P}_{q} F_{2}\right]\left(x_{2}\right)\left\|x_{1}-x_{2}\right\|^{-2 s} \mathrm{~d} x_{1} \mathrm{~d} x_{2} .
$$

It is important that the distance on the sphere is the Euclidean one. Apply the identity to $F_{j}(b)=\left[\eta_{j}, \bar{b}\right]^{-(n-1-s)}$, $j=1,2$. We obtain

$$
\begin{aligned}
K_{s}\left(\eta_{1}, \eta_{2}\right) & =(2 \gamma(s, n))^{-1} \\
& \times \int_{\mathbb{R}^{n-1}} \int_{\mathbb{R}^{n-1}} J_{\mathcal{P}}\left(x_{1}\right)^{1 / q}\left[\eta_{1}, \mathcal{P}\left(x_{1}\right)\right]^{-(n-1-s)} J_{\mathcal{P}}\left(x_{2}\right)^{1 / q}\left[\eta_{2}, \mathcal{P}\left(x_{2}\right)\right]^{-(n-1-s)}\left\|x_{1}-x_{2}\right\|^{-2 s} \mathrm{~d} x_{1} \mathrm{~d} x_{2} .
\end{aligned}
$$

Next, let $\alpha=\frac{n-1}{2}+s \in\left(\frac{n-1}{2}, n-1\right)$ and write the classical identity

$$
\left\|x_{1}-x_{2}\right\|^{-2 s}=\left\|x_{1}-x_{2}\right\|^{n-1-2 \alpha}=Z \int_{\mathbb{R}^{n-1}}\left\|x-x_{1}\right\|^{-\alpha}\left\|x-x_{2}\right\|^{-\alpha} \mathrm{d} x,
$$

where

$$
Z=Z(s, n)=\frac{\Gamma\left(\frac{n-1}{2}-s\right) \Gamma\left(\frac{n-1}{4}+\frac{s}{2}\right)^{2}}{\Gamma(s) \Gamma\left(\frac{n-1}{4}-\frac{s}{2}\right)^{2} \pi^{\frac{n-1}{2}}} .
$$

(Note that the integral on the right hand side converges exactly for $\alpha \in\left(\frac{n-1}{2}, n-1\right)$ ), plug it in the formula for $K_{s}$ and apply Fubini theorem (all functions are positive) ${ }^{4}$. We get

$$
K_{s}\left(\eta_{1}, \eta_{2}\right)=\int_{\mathbb{R}^{n-1}} m_{\eta_{1}}(x) m_{\eta_{2}}(x) \mathrm{d} x
$$

where

$$
m_{\eta}(x)=(2 \gamma(s, n))^{-1 / 2} Z^{1 / 2} \int_{\mathbb{R}^{n-1}}\left\|x-x_{1}\right\|^{\frac{1-n}{2}-s} J_{\mathcal{P}}\left(x_{1}\right)^{1 / q}\left[\eta, \mathcal{P}\left(x_{1}\right)\right]^{-(n-1-s)} \mathrm{d} x_{1} .
$$

\footnotetext{
${ }^{4}$ By taking Fourier transforms and using formulas from Lieb and Loss $\left\|\widehat{\|\cdot\|^{-\beta}}=\frac{c_{n-1-\beta}}{c_{\beta}}\right\| \cdot \|^{\beta-(n-1)}$, we get the equation for the constants:

$$
\frac{c_{n-1-2 s}}{c_{2 s}}=Z \frac{c_{(n-1) / 2-s}^{2}}{c_{(n-1) / 2+s}^{2}}
$$

where $c_{\beta}=\frac{\Gamma(\beta / 2)}{\pi^{\beta / 2}}$. It follows

$$
Z=\frac{c_{n-1-2 s} c_{(n-1) / 2+s}^{2}}{c_{2 s} c_{(n-1) / 2-s}^{2}}=\frac{\Gamma\left(\frac{n-1}{2}-s\right) \Gamma\left(\frac{n-1}{4}+\frac{s}{2}\right)^{2}}{\Gamma(s) \Gamma\left(\frac{n-1}{4}-\frac{s}{2}\right)^{2} \pi^{\frac{n-1}{2}}} .
$$
}


Although it is not obvious from this expression, whether $m_{\eta}(\cdot) \in L^{2}\left(\mathbb{R}^{n-1}\right)$, we know that

$$
\int_{\mathbb{R}^{n-1}} m_{\eta}^{2}(x) \mathrm{d} x=K_{s}(\eta, \eta)<\infty
$$

Therefore, we obtain a white noise integral representation of our stationary field with covariance $K_{s}$, as presented in (5.8),

$$
X^{s}(\eta)=\int_{\mathbb{R}^{n-1}} m_{\eta}(x) W(\mathrm{~d} x) .
$$

We are extremely grateful to A.I. Nazarov for idea to use stereographic projection in this context.

Remark: We refer to Chapter 6 of [25] for a general concept of model of a random function which is behind the representation (5.11).

\subsubsection{Integral representation for hyperbolic plane}

Let us be more specific for dimension $n=2$. First recall the model of the Poincaré half plane and consider the map $\overline{\mathcal{P}}: \zeta \mapsto \frac{\zeta-i}{\zeta+i}$, which is one to one map from $\mathbb{P}=\{\zeta \in \mathbb{C}, \Im(\zeta)>0\}$ onto $\mathbb{D}$. One can define a Riemannian metric on $\mathbb{P}$ such that $\mathbb{P}$ is isometric to $\mathbb{D}$, and hence is another model for the hyperbolic space in dimension $n=2$. Moreover the boundary $\partial \mathbb{P}=\{\zeta \in \mathbb{C}, \Im(\zeta)=0\}$ of $\mathbb{P}$ is mapped by the inverse of the stereographic projection, still denoted by $\overline{\mathcal{P}}$, on the boundary of $\mathbb{D}$, which is $\mathbb{S}^{1}$.

Please note that in this case the isometry group of $\mathbb{P}$ is related to $S L(2, \mathbb{R})$. See chapter 6 in [1] for more details. Many problems of hyperbolic geometry can be recast in terms of representations of $S L(n, \mathbb{R})$. See the chapter by Takahashi in [9].

Let us now translate the previous formula, with the new notations. Recall (4.25)

$$
[\eta, \bar{b}]=\frac{|z-b|^{2}}{1-|z|^{2}} .
$$

If $\zeta \in \mathbb{P}$ is such that $\overline{\mathcal{P}}(\zeta)=\mathcal{I}(\eta)=z \in \mathcal{D}$ and $t \in \mathbb{R}$ such that $\overline{\mathcal{P}}(t)=b$, then

$$
\begin{aligned}
{[\eta, \bar{b}] } & =\frac{\left|\frac{\zeta-i}{\zeta+i}-\frac{t-i}{t+i}\right|^{2}}{1-\left|\frac{\zeta-i}{\zeta+i}\right|^{2}} \\
& =\frac{|\zeta-t|^{2}}{\Im \zeta\left(1+t^{2}\right)} .
\end{aligned}
$$

In dimension $n=2$, we have $q=\frac{1}{1-s}$ and the Jacobian can be rewritten as $J_{\mathcal{P}}(t)=\frac{2}{1+t^{2}}$. Then we obtain from (5.9), for $\zeta_{1}, \zeta_{2} \in \mathbb{P}$,

$$
\begin{aligned}
K_{s}\left(\zeta_{1}, \zeta_{2}\right) & =\int_{\mathbb{R} \times \mathbb{R}}\left(\frac{2}{1+t_{1}^{2}}\right)^{1-s}\left(\frac{\left|\zeta_{1}-t_{1}\right|^{2}}{\Im \zeta_{1}\left(1+t_{1}^{2}\right)}\right)^{s-1}\left(\frac{2}{1+t_{2}^{2}}\right)^{1-s}\left(\frac{\left|\zeta_{2}-t_{2}\right|^{2}}{\Im \zeta_{2}\left(1+t_{2}^{2}\right)}\right)^{s-1}\left|t_{1}-t_{2}\right|^{-2 s} \frac{\mathrm{d} t_{1} \mathrm{~d} t_{2}}{2 \gamma(s, 2)} \\
& =\frac{2^{-s} \sqrt{\pi} \Gamma(1-s)}{\Gamma\left(\frac{1}{2}-s\right)}\left(\Im \zeta_{1}\right)^{1-s}\left(\Im \zeta_{2}\right)^{1-s} \int_{\mathbb{R}} \int_{\mathbb{R}}\left|\zeta_{1}-t_{1}\right|^{2(s-1)}\left|\zeta_{2}-t_{2}\right|^{2(s-1)}\left|t_{1}-t_{2}\right|^{-2 s} \mathrm{~d} t_{1} \mathrm{~d} t_{2} .
\end{aligned}
$$

In this case we can write the kernel in (5.10), for $\zeta \in \mathbb{P}, x \in \mathbb{R}$

$$
m_{\zeta}(x)=\left(\frac{2^{-s} \sqrt{\pi} \Gamma(1-s)}{\Gamma(1 / 2-s)}\right)^{1 / 2} Z^{1 / 2}(\Im \zeta)^{1-s} \int_{\mathbb{R}}|x-t|^{-1 / 2-s}|\zeta-t|^{2(s-1)} \mathrm{d} t .
$$


Actually there are many integral representations of $X$. We will just mention the one with respect to the white noise in the Fourier domain $\hat{W}(\mathrm{~d} u)$, because it is slightly more explicit. Let us first define $\hat{W}(d u)$ by

$$
\int f(u) \hat{W}(\mathrm{~d} u):=\int \hat{f}(x) W(\mathrm{~d} x), \quad \forall f \in L^{2}(\mathbb{R}) \text { such that } f(-u)=\overline{f(u)}
$$

where

$$
\hat{f}(u)=\int_{\mathbb{R}^{n}} \mathrm{e}^{-2 \pi \mathrm{i}(u, x)} f(x) \mathrm{d} x,
$$

in any dimension $n$. Then

$$
X^{s}(\zeta)=\int_{\mathbb{R}} \widehat{m}_{\zeta}(u) \hat{W}(\mathrm{~d} u)
$$

where

$$
\widehat{m}_{\zeta}(u)=\left(\frac{2^{-s} \sqrt{\pi} \Gamma(1-s)}{\Gamma(1 / 2-s)}\right)^{1 / 2} Z^{1 / 2}(\Im \zeta)^{1-s} \mid \widehat{\left.\left.\right|^{-(1 / 2}+s\right)}(u) \cdot\left(\left(\Re \zeta-t \widehat{)^{2}+\Im}(\zeta)^{2}\right)^{s-1}(u) .\right.
$$

Indeed, $m_{\zeta}$ is defined in (5.14) by a convolution, which corresponds to a product in (5.16).

By using

$$
\left.\left|\widehat{\mid-(1 / 2+s)}(u)=\frac{\Gamma(1 / 4-s / 2) \pi^{1 / 4+s / 2}}{\Gamma(1 / 4+s / 2) \pi^{1 / 4-s / 2}}\right| u\right|^{s-1 / 2}=\frac{\Gamma(1 / 4-s / 2) \pi^{s}}{\Gamma(1 / 4+s / 2)}|u|^{s-1 / 2},
$$

and plugging in

$$
Z=Z(s, 2)=\frac{\Gamma(1 / 2-s) \Gamma(1 / 4+s / 2)^{2}}{\Gamma(s) \Gamma(1 / 4-s / 2)^{2} \pi^{1 / 2}}
$$

we obtain

$$
\begin{aligned}
\widehat{m}_{\zeta}(u) & =\left(\frac{2^{-s} \sqrt{\pi} \Gamma(1-s)}{\Gamma(1 / 2-s)}\right)^{1 / 2} Z^{1 / 2}(\Im \zeta)^{1-s} \frac{\Gamma(1 / 4-s / 2) \pi^{s}}{\Gamma(1 / 4+s / 2)}|u|^{s-1 / 2}\left((\Re \zeta-t)^{2}+\Im(\zeta)^{2}\right)^{s-1}(u) \\
& =\left(\frac{\pi^{2 s} \Gamma(1-s)}{2^{s} \Gamma(s)}\right)^{1 / 2}(\Im \zeta)^{1-s}|u|^{s-1 / 2}\left((\Re \zeta-t)^{2+}+\Im(\zeta)^{2}\right)^{s-1}(u) .
\end{aligned}
$$

Next, one can express the Fourier transform $\left((\Re \zeta-t)^{2}+\Im(\zeta)^{2}\right)^{s-1}(u)$ via modified Bessel functions of the second kind $K$. First,

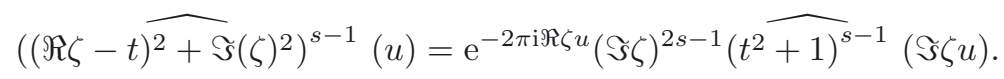

The expression for the remaining Fourier transform easily follows from the classical representation of the Modified Bessel function of the second kind $K_{\nu}$. See 8.432 1., p. 907 of [15].

$$
K_{\nu}(z)=\frac{\Gamma(\nu+1 / 2)(2 z)^{\nu}}{\sqrt{\pi}} \int_{0}^{\infty} \frac{\cos t \mathrm{~d} t}{\left(t^{2}+z^{2}\right)^{\nu+1}},
$$

which is clearly equivalent to

$$
\left(t^{2}+\widehat{\left.1)^{-(\nu}+1 / 2\right)}(u)=\frac{2 \sqrt{\pi}}{\Gamma(\nu+1 / 2)}(\pi|u|)^{\nu} K_{\nu}(2 \pi|u|) .\right.
$$

By letting $\nu=1 / 2-s$, we get

$$
\left(t^{2+1)^{s}}-1(u)=\frac{2 \sqrt{\pi}}{\Gamma(1-s)}(\pi|u|)^{1 / 2-s} K_{1 / 2-s}(2 \pi|u|) .\right.
$$


Hence we get from (5.17)

$$
\begin{aligned}
\widehat{m}_{\zeta}(u)= & \left(\frac{\pi^{2 s} \Gamma(1-s)}{2^{s} \Gamma(s)}\right)^{1 / 2}(\Im \zeta)^{1-s}|u|^{s-1 / 2} \cdot \mathrm{e}^{-2 \pi \mathrm{i} \Re \zeta u}(\Im \zeta)^{2 s-1} \cdot \frac{2 \sqrt{\pi}}{\Gamma(1-s)} \\
& \times(\pi \Im \zeta|u|)^{1 / 2-s} K_{1 / 2-s}(2 \pi \Im \zeta|u|) \\
= & \left(\frac{2^{2-s} \pi^{2}}{\Gamma(s) \Gamma(1-s)}\right)^{1 / 2}(\Im \zeta)^{\frac{1}{2}} \mathrm{e}^{-2 \pi \mathrm{i} \Re \zeta u} K_{\frac{1}{2}-s}(2 \pi \Im \zeta|u|) .
\end{aligned}
$$

\subsection{Fields with stationary increments}

Let us come back to general results and use notations of the beginning of Section 5 . We say that a complex valued random field $\left\{X_{t}, t \in T\right\}$ is a process with stationary increments in a wide sense starting at $o$ if $X_{o}=0$, $\mathbb{E} X_{t}=0$ for all $t \in T$ and $\forall t_{1}, t_{2} \in T, g \in G$

$$
\operatorname{cov}\left(X_{g t_{1}}-X_{g o}, X_{g t_{2}}-X_{g o}\right)=\operatorname{cov}\left(X_{t_{1}}, X_{t_{2}}\right) .
$$

In particular, we have

$$
\mathbb{E}\left|X_{g t_{1}}-X_{g t_{2}}\right|^{2}=\mathbb{E}\left|X_{t_{1}}-X_{t_{2}}\right|^{2} .
$$

The structure function of $X$ is defined as $\mathfrak{D}: T \rightarrow \mathbb{R}^{+}$by

$$
\mathfrak{D}(t)=\mathbb{E}\left|X_{t}\right|^{2}
$$

or $\tilde{\mathfrak{D}}: G \rightarrow \mathbb{R}^{+}$as $\tilde{\mathfrak{D}}(g)=\mathfrak{D}(g o)$.

Structure functions have a special property: they are of negative type. Recall that a function $\phi: T \times T \mapsto \mathbb{R}$ is of negative type if $\forall t_{1}, t_{2} \in T, \phi\left(t_{1}, t_{2}\right)=\phi\left(t_{2}, t_{1}\right) ; \phi\left(t_{1}, t_{1}\right)=0 ; \forall t_{1}, \ldots, t_{n} \in T, \forall c_{1}, \ldots, c_{n} \in \mathbb{R}$ such that $\sum_{i=1}^{n} c_{i}=0$

$$
\sum_{i=1}^{n} c_{i} c_{j} \phi\left(t_{i}, t_{j}\right) \leq 0 .
$$

Moreover, a function $\psi: G \mapsto \mathbb{R}$ is said to be of negative type if $\phi\left(g_{1}, g_{2}\right):=\psi\left(g_{2}^{-1} g_{1}\right)$ is of negative type with the previous definition.

The function $\tilde{\mathfrak{D}}$ has negative type since by $(5.19)$

$$
\begin{aligned}
\mathbb{E}\left|X_{g_{1} o}-X_{g_{2} o}\right|^{2} & =\mathbb{E}\left|X_{g_{1} o}-X_{g_{1}\left(g_{1}^{-1} g_{2}\right) o}\right|^{2}=\mathbb{E}\left|X_{o}-X_{g_{1}^{-1} g_{2} o}\right|^{2} \\
& =\mathbb{E}\left|X_{g_{1}^{-1} g_{2} o}\right|^{2}=\tilde{\mathfrak{D}}\left(g_{1}^{-1} g_{2}\right) .
\end{aligned}
$$

The function $\tilde{\mathfrak{D}}$ is also $G_{0}$-bi-invariant, since for any $g \in G, \rho \in G_{0}$

$$
\begin{gathered}
\tilde{\mathfrak{D}}(g \rho)=\mathfrak{D}(g \rho o)=\mathfrak{D}(g o)=\tilde{\mathfrak{D}}(g), \\
\tilde{\mathfrak{D}}(\rho g)=\mathbb{E}\left|X_{\rho g o}-X_{o}\right|^{2}=\mathbb{E}\left|X_{g o}-X_{o}\right|^{2}=\tilde{\mathfrak{D}}(g) .
\end{gathered}
$$

If the action of $G$ is rotationally transitive, then $\mathfrak{D}(t)$ only depends on $d(t, o)$, since if $d\left(t_{1}, o\right)=d\left(t_{2}, o\right), \rho t_{1}=t_{2}$,

$$
\mathfrak{D}\left(t_{2}\right)=\mathbb{E}\left|X_{t_{2}}\right|^{2}=\mathbb{E}\left|X_{\rho t_{1}}-X_{\rho o}\right|^{2}=\mathbb{E}\left|X_{t_{1}}-X_{o}\right|^{2}=\mathfrak{D}\left(t_{1}\right) .
$$

Recall now that any stationary field $\left\{Y_{t}, t \in T\right\}$ generates a field with stationary increments $X_{t}:=Y_{t}-Y_{o}$, since

$$
\begin{aligned}
\operatorname{cov}\left(X_{g t_{1}}-X_{g o}, X_{g t_{2}}-X_{g o}\right) & =\operatorname{cov}\left(Y_{g t_{1}}-Y_{g o}, Y_{g t_{2}}-Y_{g o}\right) \\
& =\operatorname{cov}\left(Y_{t_{1}}-Y_{o}, Y_{t_{2}}-Y_{o}\right)=\operatorname{cov}\left(X_{t_{1}}, X_{t_{2}}\right) .
\end{aligned}
$$


If $K(\cdot)$, the covariance of $Y$, is real, we get for the structure function of $X$,

$$
\mathfrak{D}(t)=\mathbb{E}\left|Y_{t}-Y_{o}\right|^{2}=2[K(o)-K(t)]
$$

The structure function of $X$ is bounded, thus any field with stationary increments and unbounded structure function can not be generated in this way.

Lévy-Khinchin-kind theorems enable to select the "basic" structure functions and represent arbitrary structure function as a mixture of the primary ones. For the case $T=\mathcal{L}_{n}, G$ being the group of linear transformations of $\mathbb{R}^{n+1}$ preserving the quadratic form $[\cdot, \cdot]$ (and actually for a much more general case) the following FarautHarzallah theorem accomplishes this task by using the spherical functions and the generalized quadratic form $Q$ defined in (4.10) as the primary ones [11], p. 184, p. 208.

Theorem 5.5. Let $\mathfrak{D}: \mathcal{L}_{n} \rightarrow \mathbb{R}^{+}$be a structure function of a stochastically continuous field with stationary increments. Then there exists a unique measure $\nu$ on the spectral set $\mathcal{S}$ and $c \geq 0$ such that

$$
\mathfrak{D}(\eta)=c Q(\eta)+\int_{S}\left[1-\omega_{s}(\eta)\right] \nu(\mathrm{d} s), \quad \eta \in \mathcal{L}_{n}
$$

and

$$
\int_{\mathcal{S}}(|s| \wedge 1) \nu(\mathrm{d} s)<\infty
$$

Conversely, any function of type (5.21) is a structure function of a field with stationary increments.

The measure $\nu$ is called spectral measure for any field with structure function $\mathfrak{D}$.

The second term in (5.21) corresponds to a mixture of fields arising from the stationary fields related to the covariances $\omega_{s}$.

We will now show that in asymptotic setting, as $\eta$ goes to infinity, the $Q$-term, with its linear growth, is always dominating. We state this as a proposition.

Proposition 5.6. Let $\nu$ be a measure on $\mathcal{S}$ satisfying assumption (5.22). Then

$$
\lim _{r=d_{n}(\eta, 0) \rightarrow \infty} r^{-1} \int_{\mathcal{S}}\left[1-\omega_{s}(\eta)\right] \nu(\mathrm{d} s)=0 .
$$

Proof. Since the measure $\nu$ is finite on $\{s \in \mathcal{S}:|s|>(n-1) / 2\}$, it is clear that

$$
r^{-1} \int_{|s|>(n-1) / 2}\left[1-\omega_{s}(\eta)\right] \nu(\mathrm{d} s) \leq r^{-1} \nu\{s \in \mathcal{S}:|s|>(n-1) / 2\} \rightarrow 0 .
$$

Consider the remaining part. By using (4.15), for any $k>0$ and $r>\frac{2 k}{n-1}$ we have

$$
\begin{aligned}
\int_{0}^{(n-1) / 2}\left[1-\omega_{s}(\eta)\right] \nu(\mathrm{d} s) & \leq \int_{0}^{k / r} r s \nu(\mathrm{d} s)+\int_{k / r}^{(n-1) / 2} 1 \nu(\mathrm{d} s) \\
& \leq r \int_{0}^{k / r} s \nu(\mathrm{d} s)+\int_{k / r}^{(n-1) / 2} \frac{s}{k / r} \nu(\mathrm{d} s) \\
& \leq r \int_{0}^{k / r} s \nu(\mathrm{d} s)+\frac{r}{k} \int_{0}^{(n-1) / 2} s \nu(\mathrm{d} s) .
\end{aligned}
$$

It follows that

$$
\lim _{r \rightarrow \infty} r^{-1} \int_{0}^{(n-1) / 2}\left[1-\omega_{s}(\eta)\right] \nu(\mathrm{d} s) \leq \frac{1}{k} \int_{0}^{(n-1) / 2} s \nu(\mathrm{d} s)
$$

and we are done by letting $k \rightarrow \infty$. 
We finish this section by describing some transformations of the structure functions, see [11], p. 179. Define Bernstein function as

$$
F(u)=c u+\int_{0}^{\infty}\left(1-\mathrm{e}^{-s u}\right) \mu(\mathrm{d} s)
$$

where $c \geq 0$ and $\mu$ is a measure on $(0, \infty)$ such that $\int(s \wedge 1) \mu(\mathrm{d} s)<\infty$.

The main examples of Bernstein functions are

$$
F(u)=u^{\alpha}=\frac{\alpha}{\Gamma(1-\alpha)} \int_{0}^{\infty}\left(1-\mathrm{e}^{-s u}\right) \frac{\mathrm{d} s}{s^{1+\alpha}}
$$

for $0<\alpha<1$ and

$$
F(u)=\log (1+u)=\int_{0}^{\infty}\left(1-\mathrm{e}^{-s u}\right) \frac{\mathrm{e}^{-s} \mathrm{~d} s}{s} .
$$

The main property of Bernstein functions is the following: If $F$ is such a function and $\mathfrak{D}(\cdot)$ is the structure function of a field with stationary increments, then there exists a field with stationary increments and structure function $F(\mathfrak{D}(\cdot))$.

\section{Homogeneous fields BASED ON HOROCYCLES}

Assume now that $G$ acts on a space $T$ and let $G_{0}$ be a subgroup of $G$. Assume that there is a $G$-invariant measure $\Lambda$ on $T$. Take a white noise $W$ on $T$ controlled by $\Lambda$ and any $f \in L^{2}(T, \Lambda)$ which is $G_{0}$-invariant, that is $f(\rho t)=f(t)$ for all $t \in T, \rho \in G_{0}$. Define a field $\left\{X_{z}^{f}, z \in G / G_{0}\right\}$ by letting for $g \in z \in G / G_{0}$

$$
X_{z}^{f}=\int_{T} f\left(g^{-1} t\right) W(\mathrm{~d} t)
$$

This is correct, since $g_{1}, g_{2} \in z$ implies $g_{1}=g_{2} \rho$ for some $\rho \in G_{0}$, hence $g_{1}^{-1}=\rho^{-1} g_{2}^{-1}$ and $f\left(g_{1}^{-1} t\right)=$ $f\left(\rho^{-1} g_{2}^{-1} t\right)=f\left(g_{2}^{-1} t\right)$.

Moreover, $X_{z}^{f}$ is a stationary process. Indeed, for any $g_{1} \in z_{1}, g_{2} \in z_{2}$ and $g \in G$, the invariance of $\Lambda$ yields

$$
\begin{aligned}
\operatorname{cov}\left(X_{g z_{1}}^{f}, X_{g z_{2}}^{f}\right) & =\int_{T} f\left(\left(g g_{1}\right)^{-1} t\right) \bar{f}\left(\left(g g_{2}\right)^{-1} t\right) \Lambda(\mathrm{d} t) \\
& =\int_{T} f\left(g_{1}^{-1} g^{-1} t\right) \bar{f}\left(g_{2}^{-1} g^{-1} t\right) \Lambda(\mathrm{d} t) \\
& =\int_{T} f\left(g_{1}^{-1} u\right) \bar{f}\left(g_{2}^{-1} u\right) \Lambda(\mathrm{d} u) \\
& =\operatorname{cov}\left(X_{z_{1}}^{f}, X_{z_{2}}^{f}\right) .
\end{aligned}
$$

\subsection{Definitions and basic example}

We will now specify the general framework of (6.1) to the particular case of the group $G=S U(1,1)$ acting on the horocycle space $\mathcal{H}$ parameterized according to (3.12). Recall that the action is described in (3.13) and the invariant measure is given in (3.14). Take any function $f \in L^{2}\left(\mathbb{R}, \mathrm{e}^{2 r} d r\right)$ and identify it with the function on $\mathcal{H}$, $f(r, b)=f(r)$. This function will be rotation invariant, since the action of any rotation $\rho$ on $\mathcal{H}$ preserves the first coordinate: $\rho(r, b)=(r, \rho(b))$. According to the general theory, we can construct a stationary field $\left\{X_{z}^{f}, z \in \mathbb{D}\right\}$ by taking a stationary white noise $W$ controlled by invariant measure $\mathrm{d} h$ and letting

$$
X_{g(0)}^{f}=\int_{\mathcal{H}} f\left(g^{-1} h\right) W(\mathrm{~d} h) .
$$


Let $z=g(0)$. Since by (3.10) we have $\left\langle g^{-1}(0), g^{-1}(b)\right\rangle=-\langle g(0), b\rangle=-\langle z, b\rangle$, it is true that

$$
X_{z}^{f}=\int_{\mathcal{H}} f\left(r+\left\langle g^{-1}(0), g^{-1}(b)\right\rangle\right) W(\mathrm{~d} h)=\int_{\mathcal{H}} f(r-\langle z, b\rangle) W(\mathrm{~d} h)
$$

Therefore, the corresponding covariance is

$$
\operatorname{cov}\left(X_{z}^{f}, X_{0}^{f}\right)=\int_{B} \int_{-\infty}^{\infty} f(r-\langle z, b\rangle) \bar{f}(r) \mathrm{e}^{2 r} \mathrm{~d} r \mathrm{~d} b .
$$

One of the most interesting examples is stationary Chentsov horocycle process $V_{z}$ associated with the function $f(r)=1_{r \leq 0}$. We have

$$
\operatorname{cov}\left(V_{z}, V_{0}\right)=\int_{B} \int_{-\infty}^{\min \{0,\langle z, b\rangle\}} \mathrm{e}^{2 r} \mathrm{~d} r \mathrm{~d} b=\frac{1}{2} \int_{B} \exp \{2 \min \{0,\langle z, b\rangle\}\} \mathrm{d} b .
$$

It is not very difficult to calculate the covariance explicitly. Indeed, take a real $z \in[0,1)$. By using (3.17) and (3.16) we get

$$
\begin{aligned}
& \int_{B} \exp \{2 \min \{0,\langle z, b\rangle\}\} \mathrm{d} b=\int_{\langle z, b\rangle<0} \exp \{2\langle z, b\rangle\} \mathrm{d} b+\int_{\langle z, b\rangle>0} 1 \mathrm{~d} b \\
= & 1+\int_{\langle z, b\rangle>0}(1-\exp \{2\langle z, b\rangle\}) \mathrm{d} b=1+\int_{\cos b>z}(1-\exp \{2\langle z, b\rangle\}) \mathrm{d} b \\
= & 1+\int_{\cos b>z}\left(1-\frac{1-z^{2}}{|z-b|^{2}}\right) \mathrm{d} b=1+\frac{2}{2 \pi} \int_{0}^{\beta}\left(1-\frac{1-z^{2}}{\left|z-\mathrm{e}^{\mathrm{i} \varphi}\right|^{2}}\right) \mathrm{d} \varphi \\
= & 1+\frac{\beta}{\pi}-\frac{\left(1-z^{2}\right)}{\pi} \int_{0}^{\beta} \frac{\mathrm{d} \varphi}{\left|z-\mathrm{e}^{\mathrm{i} \varphi}\right|^{2}},
\end{aligned}
$$

where $\beta=\arccos z$. Next,

$$
\begin{aligned}
& \int_{0}^{\beta} \frac{\mathrm{d} \varphi}{\left|z-\mathrm{e}^{\mathrm{i} \varphi}\right|^{2}}=\int_{0}^{\beta} \frac{\mathrm{d} \varphi}{(z-\cos \varphi)^{2}+\sin ^{2} \varphi} \\
= & \int_{0}^{\beta} \frac{\mathrm{d} \varphi}{\left(z^{2}+1\right)-2 z \cos \varphi}=\int_{0}^{\beta} \frac{\mathrm{d} \varphi}{(1-z)^{2} \cos (\varphi / 2)+(1+z)^{2} \sin ^{2}(\varphi / 2)} \\
= & 2 \int_{0}^{\beta} \frac{\mathrm{d} \tan (\varphi / 2)}{(1-z)^{2}+(1+z)^{2} \tan ^{2}(\varphi / 2)}=2 \int_{0}^{\tan (\beta / 2)} \frac{\mathrm{d} t}{(1-z)^{2}+(1+z)^{2} t^{2}} \\
= & \frac{2}{1-z^{2}} \arctan \left(\frac{(1+z) \tan (\beta / 2)}{1-z}\right) .
\end{aligned}
$$

Since $\tan (\beta / 2)=\sqrt{\frac{1-z}{1+z}}$, we have $\arctan \sqrt{\frac{1+z}{1-z}}=\frac{\pi}{2}-\frac{\beta}{2}$ and we obtain

$$
\int_{0}^{\beta} \frac{\mathrm{d} \varphi}{\left|z-\mathrm{e}^{\mathrm{i} \varphi}\right|^{2}}=\frac{2}{1-z^{2}} \arctan \sqrt{\frac{1+z}{1-z}}=\frac{2}{1-z^{2}}\left(\frac{\pi}{2}-\frac{\beta}{2}\right)=\frac{\pi-\beta}{1-z^{2}} .
$$

We end up with a strikingly simple formula

$$
\operatorname{cov}\left(V_{z}, V_{0}\right)=\frac{1}{2}\left[1+\frac{\beta}{\pi}-\frac{\left(1-z^{2}\right)}{\pi} \frac{\pi-\beta}{1-z^{2}}\right]=\frac{\beta}{\pi}=\frac{\arccos z}{\pi} .
$$


For arbitrary $z \in \mathbb{D}$ we have

$$
\operatorname{cov}\left(V_{z}, V_{0}\right)=\frac{\arccos |z|}{\pi}
$$

The Chentsov horocycle field with stationary increments $W_{\mathcal{H}}(z):=V_{z}-V_{0}$ has a particularly clear geometric meaning. Indeed, let $z \in \mathbb{D}$. Then by the definition,

$$
W_{\mathcal{H}}(z)=\int_{B} \int_{-\infty}^{\infty}\left(1_{r \leq\langle z, b\rangle}-1_{r \leq 0}\right) W(\mathrm{~d} h)=W\left(A_{z}^{+}\right)-W\left(A_{z}^{-}\right),
$$

where the sets $A_{z}^{+}=\{(r, b): 0<r \leq\langle z, b\rangle\}, A_{z}^{-}=\{(r, b):\langle z, b\rangle<r \leq 0\} \subset \mathcal{H}$ are disjoint and $A_{z}:=A_{z}^{+} \cup A_{z}^{-}$ is exactly the set of horocycles separating the points 0 and $z$. This is of course an exact equivalent to Chentsov's construction of Lévy's Brownian field in $R^{n}$, [6], with horocycles instead of hyperplanes.

Finally, note that the structure function of $W_{\mathcal{H}}$ is fairly simple: it follows from (6.6) that

$$
\mathfrak{D}(z)=\mathbb{E}\left|W_{\mathcal{H}}\right|^{2}=\mathbb{E}\left|V_{z}-V_{0}\right|^{2}=2\left(\mathbb{E}\left|V_{0}\right|^{2}-\operatorname{cov}\left(V_{z}, V_{0}\right)\right)=\frac{2}{\pi}(1-\arccos |z|) .
$$

This is a bounded function. Thus $W_{\mathcal{H}}$ is not a Lévy Brownian field on $\mathbb{D}$, which is defined in Section 8 .

\subsection{Spectral representation}

We will now give a spectral representation of arbitrary stationary field based on horocycles. Take a function $f \in L^{2}\left(\mathbb{R}, \mathrm{e}^{2 r} \mathrm{~d} r\right)$ as above and consider the associated classical Fourier transform of $f(r) \mathrm{e}^{r}$

$$
F(\lambda)=\frac{1}{\sqrt{2 \pi}} \int_{-\infty}^{\infty} f(r) \mathrm{e}^{r} \mathrm{e}^{\mathrm{i} \lambda r} \mathrm{~d} r .
$$

By Plancherel formula,

$$
\begin{aligned}
\int_{-\infty}^{\infty} f(r-\langle z, b\rangle) \bar{f}(r) \mathrm{e}^{2 r} \mathrm{~d} r & =\mathrm{e}^{\langle z, b\rangle} \int_{-\infty}^{\infty} \mathrm{e}^{r-\langle z, b\rangle} f(r-\langle z, b\rangle) \bar{f}(r) \mathrm{e}^{r} \mathrm{~d} r \\
& =\mathrm{e}^{\langle z, b\rangle} \int_{-\infty}^{\infty} F(\lambda) \mathrm{e}^{\mathrm{i} \lambda\langle z, b\rangle} \bar{F}(\lambda) \mathrm{d} \lambda \\
& =\mathrm{e}^{\langle z, b\rangle} \int_{-\infty}^{\infty}|F(\lambda)|^{2} \mathrm{e}^{\mathrm{i} \lambda\langle z, b\rangle} \mathrm{d} \lambda .
\end{aligned}
$$

Hence,

$$
\begin{aligned}
\operatorname{cov}\left(X_{z}^{f}, X_{0}^{f}\right) & =\int_{B} \mathrm{e}^{\langle z, b\rangle} \int_{-\infty}^{\infty}|F(\lambda)|^{2} \mathrm{e}^{\mathrm{i} \lambda\langle z, b\rangle} \mathrm{d} \lambda \mathrm{d} b \\
& =\int_{-\infty}^{\infty}|F(\lambda)|^{2} \int_{B} \mathrm{e}^{(1+\mathrm{i} \lambda)\langle z, b\rangle} \mathrm{d} b \mathrm{~d} \lambda \\
& =\int_{-\infty}^{\infty}|F(\lambda)|^{2} \varphi_{\lambda}(z) \mathrm{d} \lambda
\end{aligned}
$$

We see that $\nu(\mathrm{d} \lambda)=2|F(\lambda)|^{2} \mathrm{~d} \lambda$ is the spectral measure for the field $X^{f}$. Any absolutely continuous spectral measure can be obtained in this way. Yet the singular spectral measures are unavailable.

In the particular case of Chentsov horocycle field we have

$$
F(\lambda)=\frac{1}{\sqrt{2 \pi}} \int_{-\infty}^{0} \mathrm{e}^{(1+\mathrm{i} \lambda) r} \mathrm{~d} r=\frac{1}{\sqrt{2 \pi}}(1+\mathrm{i} \lambda)^{-1} .
$$


Hence the spectral measure

$$
\nu(\mathrm{d} \lambda)=\frac{1}{\pi}\left(1+\lambda^{2}\right)^{-1} \mathrm{~d} \lambda
$$

is just Cauchy measure, exactly as for the classical Ornstein-Uhlenbeck process.

\section{Homogeneous fields BASED ON GEODESICS}

\subsection{Basic geometry of geodesic lines}

We consider the two-dimensional hyperbolic space $\mathbb{D}$. Let $\mathcal{G}$ denote the set of all geodesic lines in $\mathbb{D}$. Recall that any $\ell \in \mathcal{G}$ is the intersection of $\mathbb{D}$ with an Euclidean circle crossing the boundary $B$ orthogonally. As a limiting case, any diameter of $\mathbb{D}$ also is a geodesic line.

There exist different ways to parameterize the two-dimensional set $\mathcal{G}$. Let $\ell=S \cap \mathbb{D} \in \mathcal{G}$ where $S$ is an Euclidean circle generating $\ell$. Let $r$ denote the radius of $S$ and $R$ denote the Euclidean distance between 0 and the center of $S$. Finally, let $\left\{b_{1}, b_{2}\right\}=S \cap B$. We can always write

$$
b_{1}=\mathrm{e}^{\mathrm{i}(\psi+\varphi)}, \quad b_{2}=\mathrm{e}^{\mathrm{i}(\psi-\varphi)},
$$

where $\psi=\psi(\ell) \in[0,2 \pi)$ determines the "direction" of the geodesic $\ell$ and $\varphi=\varphi(\ell) \in[0, \pi / 2]$ determines its "Euclidean size".

It is easy to see that the following relations are true:

$$
r=\tan \varphi, \quad R=\sqrt{r^{2}+1}=\frac{1}{\cos \varphi} .
$$

The Euclidean distance between 0 and $\ell$ is

$$
R-r=\frac{1}{\cos \varphi}-\tan \varphi=\frac{1-\sin \varphi}{\cos \varphi}=\tan (\pi / 4-\varphi / 2) .
$$

One can parameterize $\mathcal{G}$ either with pairs $\left(b_{1}, b_{2}\right)$ or $(\varphi, \psi)$. We will mainly prefer the last option.

\subsection{Separation condition}

It will be important for us in the sequel to know which geodesic lines separate two given points. Let $z=$ $(x, y) \in \mathbb{D}$ be fixed. Let us find out which geodesics separate $z$ from 0 . To begin with, consider a geodesic line $\ell$ with $\psi=0$. It means that $\ell$ is a part of the circle of radius $r$ centered at the point $(R, 0)$. The equation of the circle is $y^{2}+(x-R)^{2}=r^{2}$, hence the separation condition is $y^{2}+(x-R)^{2}<r^{2}$ which is equivalent to

$$
|z|^{2}+R^{2}-2 x R<r^{2} .
$$

By using (7.2) this reduces to

$$
|z|^{2}+1-\frac{2 x}{\cos \varphi}<0 .
$$

For general geodesic line (with arbitrary $\psi$ ), $x$ should be replaced with respective coordinate of $z$, that is with $x \cos \psi+y \sin \psi$. We arrive at separation condition

$$
|z|^{2}+1-\frac{2 x \cos \psi+2 y \sin \psi}{\cos \varphi}<0 .
$$

In particular, for real $z=x$ we have $y=0$ and separation condition becomes

$$
\cos \varphi<\frac{2 x}{x^{2}+1} \cos \psi .
$$




\subsection{Invariant measure}

Obviously, the group of isometries $S U(1,1)$ acts on $\mathcal{G}$. It is therefore important to find an invariant measure of this action. We refer to [34] for other methods to find invariant measures for $n=2$ and generalization for $n \geq 3$. In view of rotation invariance, the invariant measure $\Lambda$ should have the form

$$
\Lambda(\mathrm{d} \ell)=p(\varphi) \mathrm{d} \varphi \mathrm{d} \psi
$$

and we just need to find the density $p(\cdot)$. The direct calculation is tedious but the following trick helps. For $u \in(0,1)$ let $\mathcal{G}_{u}$ denote the set of geodesic lines crossing the Euclidean ball of radius $u$ centered at 0 . Then by $(7.3)$

$$
\begin{aligned}
\mathcal{G}_{u} & =\{\ell=(\varphi, \psi): \tan (\pi / 4-\varphi / 2) \leq u\} \\
& =\{\ell: \pi / 4-\varphi / 2 \leq \arctan u\} \\
& =\{\ell: \pi / 2 \geq \varphi \geq \pi / 2-2 \arctan u\} .
\end{aligned}
$$

By integral geometry reasons $\Lambda\left(\mathcal{G}_{u}\right)$ should be proportional to the length of the boundary of ball calculated with the Riemann metrics of $\mathbb{D}$. By [17], p. 29, this length is equal to

$$
\int_{0}^{2 \pi}\left(1-u^{2}\right)^{-1}\left(u^{2} \sin ^{2} \varphi+u^{2} \cos ^{2} \varphi\right)^{1 / 2} \mathrm{~d} \varphi=\frac{2 \pi u}{1-u^{2}}
$$

We obtain the equation

$$
\int_{\pi / 2-2 \arctan u}^{\pi / 2} p(\varphi) \mathrm{d} \varphi=\frac{c u}{1-u^{2}} .
$$

Now easy calculation yields $p(\varphi)=\frac{c}{2 \sin ^{2} \varphi}$. Indeed,

$$
\begin{aligned}
\int_{\pi / 2-2 \arctan u}^{\pi / 2} \frac{\mathrm{d} \varphi}{\sin ^{2} \varphi} & =\int_{0}^{2 \arctan u} \frac{\mathrm{d} \varphi}{\cos ^{2} \varphi}=\tan (2 \arctan u) \\
& =\frac{\sin (2 \arctan u)}{\cos (2 \arctan u)}=\frac{2 \sin (\arctan u) \cos (\arctan u)}{\cos ^{2}(\arctan u)-\sin ^{2}(\arctan u)} \\
& =\frac{2 \tan (\arctan u)}{1-\tan ^{2}(\arctan u)}=\frac{2 u}{1-u^{2}},
\end{aligned}
$$

as required. We have obtained

$$
\Lambda(\mathrm{d} \ell)=\frac{c \mathrm{~d} \varphi \mathrm{d} \psi}{\sin ^{2} \varphi} .
$$

It is instructive to calculate the measure of geodesics separating given points. Take a real $z=x \in \mathbb{D}$ and set $\beta=\frac{2 x}{x^{2}+1}$. According to (7.6), calculate

$$
\begin{aligned}
& \int_{\mathcal{G}} 1_{\cos \varphi<\beta \cos \psi} \Lambda(\mathrm{d} \ell)=\int_{0}^{2 \pi} \int_{0}^{\pi / 2} 1_{\cos \varphi<\beta \cos \psi} \frac{c \mathrm{~d} \varphi \mathrm{d} \psi}{\sin ^{2} \varphi} \\
= & 2 c \int_{0}^{\pi / 2} \int_{\arccos (\beta \cos \psi)}^{\pi / 2} \frac{\mathrm{d} \varphi}{\sin ^{2} \varphi} \mathrm{d} \psi=2 c \int_{0}^{\pi / 2} \cot (\arccos (\beta \cos \psi)) \mathrm{d} \psi \\
= & 2 c \int_{0}^{\pi / 2} \frac{\beta \cos \psi}{\sqrt{1-\beta^{2} \cos ^{2} \psi}} \mathrm{d} \psi=2 c \int_{0}^{\pi / 2} \frac{\beta \cos \psi}{\sqrt{\left(1-\beta^{2}\right)+\beta^{2} \sin ^{2} \psi}} \mathrm{d} \psi \\
= & 2 c \int_{0}^{1} \frac{\beta \mathrm{d} s}{\sqrt{\left(1-\beta^{2}\right)+\beta^{2} s^{2}}}=2 c \int_{0}^{1} \frac{\mathrm{d} s}{\sqrt{a^{2}+s^{2}}},
\end{aligned}
$$


where

$$
a^{2}=\frac{1-\beta^{2}}{\beta^{2}}=\frac{1}{\beta^{2}}-1=\frac{\left(x^{2}+1\right)^{2}}{4 x^{2}}-1=\frac{\left(1-x^{2}\right)^{2}}{4 x^{2}},
$$

hence $a=\frac{1-x^{2}}{2 x}$ and, by using (3.2),

$$
\begin{aligned}
& \int_{\mathcal{G}} 1_{\cos \varphi<\beta} \cos \psi \Lambda(\mathrm{d} \ell)=2 c \int_{0}^{1 / a} \frac{\mathrm{d} v}{\sqrt{1+v^{2}}} \\
& =2 c \log \left(\frac{1}{a}+\sqrt{\frac{1}{a^{2}}+1}\right)=2 c \log \left(\frac{1+\sqrt{a^{2}+1}}{a}\right) \\
& =2 c \log \left(\frac{1+1 / \beta}{a}\right)=2 c \log \left(\frac{\beta+1}{\beta a}\right) \\
& =2 c \log \frac{1+x}{1-x}=4 c d(0, z) \text {. }
\end{aligned}
$$

We see that the measure of separating set is proportional to the distance between the separated points. By transitivity of the action this conclusion remains true for any pair of points in $\mathbb{D}$.

\subsection{Action of isometries}

Any isometry $g$ acts in a natural way on $\mathcal{G}$ since the image of a geodesic line is again a geodesic line. We need a parametric interpretation for this action. We start with fixing an isometry $g$ and a geodesic line $\ell$ with endpoints $b_{1}, b_{2}$ as stated in (7.1). Then $g(\ell)$ is a geodesic line with endpoints

$$
\tilde{b}_{1}=g\left(b_{1}\right):=\mathrm{e}^{\mathrm{i}(\tilde{\psi}+\tilde{\varphi})}, \quad \tilde{b}_{2}=g\left(b_{2}\right):=\mathrm{e}^{\mathrm{i}(\tilde{\psi}-\tilde{\varphi})} .
$$

By using differentiation formula $(3.11)$ we see that the Jacobian of the map $\left(b_{1}, b_{2}\right) \rightarrow\left(g\left(b_{1}\right), g\left(b_{2}\right)\right)$ is

$$
J\left(b_{1}, b_{2}\right)=\exp \left\{-2\left\langle g(0), g\left(b_{1}\right)\right\rangle-2\left\langle g(0), g\left(b_{2}\right)\right\rangle\right\} \text {. }
$$

By using the expression of invariant measure (7.7) we have

$$
\frac{1}{\sin ^{2} \tilde{\varphi}}=\frac{1}{\sin ^{2} \varphi}\left|J\left(b_{1}, b_{2}\right)\right|^{-1}=\frac{1}{\sin ^{2} \varphi} \exp \left\{2\left\langle g(0), g\left(b_{1}\right)\right\rangle+2\left\langle g(0), g\left(b_{2}\right)\right\rangle\right\} \text {. }
$$

We rewrite this as

$$
\sin ^{2} \varphi=\sin ^{2} \tilde{\varphi} \exp \left\{2\left\langle g(0), g\left(b_{1}\right)\right\rangle+2\left\langle g(0), g\left(b_{2}\right)\right\rangle\right\} .
$$

\subsection{Stationary fields based on geodesics lines}

According to general rule (6.1), we can define a stationary field by

$$
X_{g(0)}^{f}=\int_{\mathcal{G}} f\left(g^{-1} \ell\right) W(\mathrm{~d} \ell) .
$$

Since $f$ must be rotation invariant, we have

$$
f(\ell)=f(\varphi, \psi)=f(\varphi):=F\left(\sin ^{2} \varphi\right) .
$$

Giving a noise a coordinate form, our field becomes

$$
X_{g(0)}^{F}=\int_{0}^{2 \pi} \int_{0}^{\pi / 2} F\left(\sin ^{2} \varphi_{0}(g, \varphi, \psi)\right) W(\mathrm{~d} \varphi \mathrm{d} \psi)
$$


where $\varphi_{0}(g, \varphi, \psi)$ is the $\varphi$-coordinate of the geodesic line $g^{-1}(\ell)$. It follows from (7.10) that

$$
\sin ^{2} \varphi_{0}(g, \varphi, \psi)=\sin ^{2} \varphi \exp \left\{2\left\langle g(0), b_{1}\right\rangle+2\left\langle g(0), b_{2}\right\rangle\right\} .
$$

Hence, for $z=g(0)$,

$$
X_{z}^{F}=\int_{0}^{2 \pi} \int_{0}^{\pi / 2} F\left(\sin ^{2} \varphi \exp \left\{2\left\langle z, b_{1}\right\rangle+2\left\langle z, b_{2}\right\rangle\right\}\right) W(\mathrm{~d} \varphi \mathrm{d} \psi)
$$

Recall that the control measure for the white noise is given by (7.7). Therefore, the field is well defined iff

$$
\int_{0}^{\pi / 2}\left|F\left(\sin ^{2} \varphi\right)\right|^{2} \frac{\mathrm{d} \varphi}{\sin ^{2} \varphi}<\infty \Leftrightarrow \int_{0}^{1} \frac{|F(v)|^{2} \mathrm{~d} v}{v^{3 / 2}(1-v)^{1 / 2}}<\infty .
$$

Finally, for covariance we get

$$
\operatorname{cov}\left(X_{z}^{F}, X_{0}^{F}\right)=c^{2} \int_{0}^{2 \pi} \int_{0}^{\pi / 2} F\left(\sin ^{2} \varphi \exp \left\{2\left\langle z, b_{1}\right\rangle+2\left\langle z, b_{2}\right\rangle\right\}\right) \overline{F\left(\sin ^{2} \varphi\right)} \frac{\mathrm{d} \varphi \mathrm{d} \psi}{\sin ^{2} \varphi} .
$$

A nice example is given by $F(v)=v^{\beta}$ when $\beta>1 / 4$. Then

$$
\operatorname{cov}\left(X_{z}^{F}, X_{0}^{F}\right)=c^{2} \int_{0}^{2 \pi} \int_{0}^{\pi / 2} \sin ^{4 \beta-2} \varphi \exp \left\{2 \beta\left\langle z, b_{1}\right\rangle+2 \beta\left\langle z, b_{2}\right\rangle\right\} \mathrm{d} \varphi \mathrm{d} \psi
$$

\section{LÉvy BRownian field AND FRACTIONAL Brownian FIELDS}

\subsection{General definitions}

Let $(T, d)$ be a metric space with a marked point $o$. A centered real Gaussian field $\left\{W_{t}, t \in T\right\}$ is called Lévy Brownian field if

$$
\operatorname{cov}\left(W_{t_{1}}, W_{t_{2}}\right)=\frac{1}{2}\left(d\left(t_{1}, o\right)+d\left(t_{2}, o\right)-d\left(t_{1}, t_{2}\right)\right)
$$

which is equivalent to $W_{o}=0$ and

$$
\mathbb{E}\left(W_{t_{1}}-W_{t_{2}}\right)^{2}=d\left(t_{1}, t_{2}\right)
$$

We refer to $[4,24]$ for more information about Lévy Brownian fields on normed spaces.

More generally, given $H>0$, a centered real Gaussian field $\left\{W_{t}^{H}, t \in T\right\}$ is called $H$-fractional Lévy Brownian field if

$$
\operatorname{cov}\left(W_{t_{1}}^{H}, W_{t_{2}}^{H}\right)=\frac{1}{2}\left(d\left(t_{1}, o\right)^{2 H}+d\left(t_{2}, o\right)^{2 H}-d\left(t_{1}, t_{2}\right)^{2 H}\right)
$$

which is equivalent to $W_{o}^{H}=0$ and

$$
\mathbb{E}\left(W_{t_{1}}^{H}-W_{t_{2}}^{H}\right)^{2}=d\left(t_{1}, t_{2}\right)^{2 H} .
$$

The existence of such fields is a hard problem depending on the properties of $T$. In general, by using Bernstein function $F(u)=u^{\alpha}, \alpha<1$, we can only say that the existence of $W^{H}$ implies the existence of $W^{H^{\prime}}$ for all $0<H^{\prime}<H$.

For example, it is known that for $T=R^{n}$ the fractional Lévy Brownian field exists whenever $0<H \leq 1$. For further discussions and examples on this topic see $[18,19]$

In the following, we consider the hyperbolic case $T=\mathcal{L}_{n}$ and $d=d_{n}$ being invariant metrics, as defined in (4.1). Lévy Brownian field for hyperbolic case was considered in [28-30,38]. Moreover, it is easily seen that in this case $W^{H}$ should be a field with stationary increments and structure function

$$
\mathfrak{D}(\eta)=\mathbb{E}\left|W_{\eta}^{H}\right|^{2}=d(\eta, \overline{0})^{2 H} .
$$


As we see from (4.34) and Proposition 5.6 no structure function can increase faster than $d(\eta, \overline{0})$. Therefore, $W^{H}$ does not exist on hyperbolic space when $H>1 / 2$. Conversely, Faraut [11], p. 205, proves that Lévy Brownian field does exist. Therefore, fractional Lévy Brownian field exists iff $0<H \leq 1 / 2$.

Recall Chentsov representation for Lévy Brownian field. Consider again the set of all geodesic lines $\mathcal{G}$ equipped with invariant measure $\Lambda$ from (7.7) and let $W$ be a white noise controlled by $\Lambda$, as considered in (7.11). For $z_{1}, z_{2} \in \mathbb{D}$, let $\mathcal{G}_{z_{1}, z_{2}} \subset \mathcal{G}$ be the set of all geodesic lines separating $z_{1}$ from $z_{2}$. We know from (7.8) that $\Lambda\left(\mathcal{G}_{z_{1}, z_{2}}\right)=4 c d\left(z_{1}, z_{2}\right)$. Consider a random field

$$
X_{z}=(4 c)^{-1 / 2} W\left(\mathcal{G}_{z, 0}\right), \quad z \in \mathbb{D} .
$$

Then

$$
\mathbb{E}\left|X_{z_{1}}-X_{z_{2}}\right|^{2}=(4 c)^{-1} \Lambda\left(\mathcal{G}_{z_{1}, 0} \Delta \mathcal{G}_{z_{2}, 0}\right)=(4 c)^{-1} \Lambda\left(\mathcal{G}_{z_{1}, z_{2}}\right)=d\left(z_{1}, z_{2}\right) .
$$

Hence, $X$ is a Lévy Brownian field.

A similar construction is available in higher dimensions $n>2$. One only has to replace separating geodesic lines with appropriate surfaces of codimension one. The details are given in [32], along with a discussion of problems arising when one tries to extend the Chentsov construction to complex hyperbolic spaces or to projective spaces.

In Euclidean case, Chentsov construction also works via separating points by straight lines (or hyperplanes, if $n>2$ ). Moreover, Takenaka [37] gave a similar construction for fractional Lévy Brownian field on $\mathbb{R}^{n}$ by using separating hyperspheres. We do not know how to extend his construction to the sphere or to hyperbolic space.

\subsection{Spectral properties}

Let us now consider the spectral decompositions. For Lévy Brownian field write

$$
r:=d(0, \eta)=c Q(\eta)+\int_{\mathcal{S}}\left[1-\omega_{s}(\eta)\right] \nu(\mathrm{d} s), \quad \eta \in \mathcal{L}_{n} .
$$

By using (4.34) and Proposition 5.6 we see that

$$
1=c \lim _{r \rightarrow \infty} \frac{Q(\eta)}{r}+\lim _{r \rightarrow \infty} r^{-1} \int_{\mathcal{S}}\left[1-\omega_{s}(\eta)\right] \nu(\mathrm{d} s)=c .
$$

Hence, the $Q$-term is really present. Consider now the remainder,

$$
I(\eta)=r-Q(\eta)=\int_{\mathcal{S}}\left[1-\omega_{s}(\eta)\right] \nu(\mathrm{d} s)
$$

We know from (4.34) that $I(\eta)=\tilde{C}_{n}-q_{n}(\eta)$. We show now that $\nu$ is a finite measure. Take any positive $\varepsilon<\frac{n-1}{2}$ and set $\mathcal{S}_{\varepsilon}=\left[\varepsilon, \frac{n-1}{2}\right]$. Since $\omega_{s}(\eta)$ is decreasing to zero uniformly on $s \in \mathcal{S}_{\varepsilon}$ when $r=d_{n}(\eta, \overline{0}) \rightarrow \infty$ and also $q_{n}(\eta)$ tends to zero, we have

$$
\tilde{C}_{n} \geq \lim _{r \rightarrow \infty} \int_{\mathcal{S}_{\varepsilon}}\left[1-\omega_{s}(\eta)\right] \nu(\mathrm{d} s)=\nu\left(\mathcal{S}_{\varepsilon}\right) .
$$

It follows that

$$
\nu\left(0, \frac{n-1}{2}\right]=\lim _{\varepsilon \rightarrow 0} \nu\left(\mathcal{S}_{\varepsilon}\right) \leq \tilde{C}_{n}<\infty .
$$


Therefore, we can split the integral in (8.2). Since $\lim _{r \rightarrow \infty} \int_{\mathcal{S}_{\varepsilon}} \omega_{s}(\eta) \nu(\mathrm{d} s)=0$, and since $\omega_{s}$ bounded implies $\lim _{r \rightarrow \infty} \int_{0}^{\varepsilon} \omega_{s}(\eta) \nu(\mathrm{d} s)=0$, we have $\lim _{r \rightarrow \infty} \int_{\mathcal{S}} \omega_{s}(\eta) \nu(\mathrm{d} s)=0$. Then

$$
\nu(S)-\int_{\mathcal{S}} \omega_{s}(\eta) \nu(\mathrm{d} s)=\tilde{C}_{n}-q_{n}(\eta)
$$

and $\lim _{r \rightarrow \infty} q_{n}(\eta)=0$ implies

$$
\int_{\mathcal{S}} \omega_{s}(\eta) \nu(\mathrm{d} s)=q_{n}(\eta)
$$

Let us now show that this indicates the absence of the lower spectrum, at least for $n \leq 5$. Denote $\mathcal{S}_{i}=\{s=$ $\left.\frac{n-1}{2}+\mathrm{i} \lambda\right\}$ the upper spectrum. Since $\omega_{s}$ are non-negative for real $s$, for any $\varepsilon<\frac{n-1}{2}$ we can write, using (4.30)

$$
\begin{aligned}
\int_{\varepsilon / 2}^{\varepsilon} \omega_{s}(\eta) \nu(\mathrm{d} s) & \leq q_{n}(\eta)+\int_{\mathcal{S}_{i}}\left|\omega_{s}(\eta)\right| \nu(\mathrm{d} s) \leq q_{n}(\eta)+\nu\left(\mathcal{S}_{i}\right) \max _{s \in \mathcal{S}_{i}}\left|\omega_{s}(\eta)\right| \\
& \leq q_{n}(\eta)+\nu\left(\mathcal{S}_{i}\right)\left|\omega_{\frac{n-1}{2}}(\eta)\right| .
\end{aligned}
$$

For $n \leq 5$ we have, by using (4.29) and (4.35)

$$
q_{n}(\eta)+\nu\left(\mathcal{S}_{i}\right)\left|\omega_{\frac{n-1}{2}}(\eta)\right|=O\left(r \mathrm{e}^{\frac{-(n-1) r}{2}}\right),
$$

while by $(4.27)$

$$
\int_{\varepsilon / 2}^{\varepsilon} \omega_{s}(\eta) \nu(\mathrm{d} s) \succeq \nu[\varepsilon / 2, \varepsilon] \cdot \mathrm{e}^{-\varepsilon r} .
$$

Since $\varepsilon<\frac{n-1}{2}$, we conclude that $\nu[\varepsilon / 2, \varepsilon]=0$. Since $\varepsilon<\frac{n-1}{2}$ was chosen arbitrary, it follows that $\nu\left(0, \frac{n-1}{2}\right)=0$.

For $n>5$, the same reasoning shows that there is no spectral mass in a neighborhood of zero, namely $\nu(0,2)=0$.

For $H$-fractional Lévy Brownian field, $H<1 / 2$, write

$$
r^{2 H}:=d(0, \eta)^{2 H}=c Q(\eta)+\int_{\mathcal{S}}\left[1-\omega_{s}(\eta)\right] \nu(\mathrm{d} s), \quad \eta \in \mathcal{L}_{n} .
$$

Again, by using (4.34) and Proposition 5.6 we see that

$$
0=\lim _{r \rightarrow \infty} \frac{r^{2 H}}{r}=c \lim _{r \rightarrow \infty} \frac{Q(\eta)}{r}+\lim _{r \rightarrow \infty} r^{-1} \int_{\mathcal{S}}\left[1-\omega_{s}(\eta)\right] \nu(\mathrm{d} s)=c .
$$

Hence, the $Q$-term is absent. Unlike the case $H=\frac{1}{2}$ the spectral measure should be infinite, hence it charges any neighborhood of 0 . In Section 9 we will give a representation of the spectrum in $H$-fractional case, as a mixture of the known spectra. In particular we show that $\nu\left[\varepsilon, \frac{n-1}{2}\right) \approx \varepsilon^{-2 H}$, as $\varepsilon \rightarrow 0$.

A form of the spectral representation for Lévy Brownian field, $H=1 / 2$, was found by Molchan in [28]. The upper spectrum has a density while the lower spectrum is concentrated on even integer points of the interval $\left[2, \frac{n-1}{2}\right)$. Again we see that in any dimension there is a neighborhood of zero, namely, $[0,2)$, which is not charged by the spectral measure and there is no lower spectrum at all for $n \leq 5$.

In our notation, Molchan's formula for the spectral density is

$$
p(\lambda)=\frac{\Gamma\left(\frac{n+1}{2}\right)}{2^{n} \pi^{3 / 2} \Gamma(n / 2)}\left|\Gamma\left(\frac{n-1}{2}+\mathrm{i} \lambda\right)\right|^{2} \lambda \sinh (\pi \lambda) \frac{\left|\Gamma\left(-\frac{n-1}{4}+\mathrm{i} \lambda / 2\right)\right|^{2}}{\left|\Gamma\left(\frac{n+3}{4}+\mathrm{i} \lambda / 2\right)\right|^{2}} .
$$


Some further simplifications are available for odd $n \geq 3$. By standard properties of $\Gamma$-function, one arrives to a rational function as a spectral density

$$
p(\lambda)=\frac{\Gamma\left(\frac{n+1}{2}\right)}{2^{n} \pi^{1 / 2} \Gamma(n / 2)} \prod_{j=0}^{\frac{n-3}{2}}\left[\left(\frac{n-2 j-3}{2}\right)^{2}+\lambda^{2}\right] \prod_{j=0}^{\frac{n-1}{2}}\left[\left(\frac{n-4 j-1}{4}\right)^{2}+\lambda^{2} / 4\right]^{-1}
$$

The density is decreasing as $\lambda^{-2}$ at infinity. In the simplest case, $n=3$,

$$
p(\lambda)=\frac{4 \lambda^{2}}{\pi\left(1+\lambda^{2}\right)^{2}}
$$

For the weights of the lower spectrum, [28] contains only some hints. Using these ideas one can prove that the spectral weight $w_{m}$ at even integer point $m \in\left[2, \frac{n-1}{2}\right.$ ) is (according to [31])

$$
\begin{aligned}
w_{m} & =\frac{n-1-2 m}{(n-1-m) m} \frac{\Gamma\left(1-\frac{n}{2}\right)}{\Gamma\left(1+\frac{m-n}{2}\right)} \cdot \frac{\Gamma\left(\frac{n+1}{2}\right)}{\Gamma\left(\frac{n+1}{2}-\frac{m}{2}\right)} \cdot \frac{\Gamma\left(\frac{1}{2}\right)}{\Gamma\left(\frac{1-m}{2}\right)} \cdot \frac{1}{\Gamma\left(1+\frac{m}{2}\right)} \\
& =\frac{n-1-2 m}{(n-1-m) m} \frac{\left(\begin{array}{c}
\frac{n-1}{2} \\
\frac{m}{2}
\end{array}\right)^{2}}{\left(\begin{array}{c}
n-1 \\
m
\end{array}\right)},
\end{aligned}
$$

where as usual $\left(\begin{array}{c}x \\ m\end{array}\right)=\frac{x(x-1) \cdots(x-n+1)}{m !}$.

\subsection{Spectral density of the Lévy Brownian field on hyperbolic plane}

We restrict us to the case $n=2$ and accept in this subsection Helgason's notation. Let us prove the following result.

Theorem 8.1. The spectral decomposition of the Brownian field on the hyperbolic plane is

$$
r=\frac{1}{2} Q(r)+\int_{0}^{\infty}\left[1-\varphi_{\lambda}(r)\right] p(\lambda) \mathrm{d} \lambda,
$$

where the spectral density $p$ is given by

$$
p(\lambda)=\frac{\hat{\phi}_{2}(\lambda) \lambda \tanh (\pi \lambda / 2)}{\sqrt{2 \pi}\left(\lambda^{2}+1\right)}
$$

where $\hat{\phi}_{2}$ is the classical Fourier transform of the function

$$
\phi_{2}(u)=\int_{0}^{\pi / 2} \frac{2 \sin ^{2} \theta-1}{\sqrt{\cosh ^{2} u-\sin ^{2} \theta}} \mathrm{d} \theta .
$$

Proof. Since we know that there is no lower spectrum, we can write Lévy-Khinchin formula as

$$
r=\frac{1}{2} Q(r)+\int_{0}^{\infty} p(\lambda)\left[1-\varphi_{\lambda}(z)\right] \mathrm{d} \lambda
$$

where $Q(r)=2 \log \cosh r$ is the generalized quadratic form and $p(\cdot)$ denotes the unknown spectral density. Recall that the variables $r \geq 0$ and $z \in \mathbb{D}$ are related by $r=d(z, 0)$. Apply the Helgason's version of LaplaceBeltrami operator (3.6) to both sides. Clearly, $\Delta r=2 \operatorname{coth}(2 r)$ and we know that $\Delta Q \equiv 4$. Indeed, in Faraut 
interpretation, $\Delta Q=n-1=1$ but we remember that Helgason's operator is four times larger. Anyway, it is easy to check this directly. We obtain the equation

$$
2(\operatorname{coth}(2 r)-1)=\int_{0}^{\infty}\left(\lambda^{2}+1\right) p(\lambda) \varphi_{\lambda}(z) \mathrm{d} \lambda .
$$

Notice that the left hand side is decreasing as $\mathrm{e}^{-4 r}$ at infinity, hence it belongs to $L^{2}(\mathbb{D})$ and we can apply Fourier transform defined in (3.20) in its radial version.

Letting $f(z)=2(\operatorname{coth}(2 r)-1)$, we can compare the inversion formula (3.22)

$$
f(z)=\frac{1}{2 \pi} \int_{0}^{\infty} \tilde{f}(\lambda) \lambda \tanh (\pi \lambda / 2) \varphi_{\lambda}(z) \mathrm{d} \lambda
$$

with equation above and see that

$$
\left(\lambda^{2}+1\right) p(\lambda)=\frac{1}{2 \pi} \tilde{f}(\lambda) \lambda \tanh (\pi \lambda / 2)
$$

hence

$$
p(\lambda)=\frac{\tilde{f}(\lambda) \lambda \tanh (\pi \lambda / 2)}{2 \pi\left(\lambda^{2}+1\right)} .
$$

One can think of using series representation

$$
f(z)=2(\operatorname{coth}(2 r)-1)=\frac{4 \mathrm{e}^{-2 r}}{\mathrm{e}^{2 r}-\mathrm{e}^{-2 r}}=4 \mathrm{e}^{-4 r} \frac{1}{1-\mathrm{e}^{-4 r}}=4 \sum_{k=1}^{\infty} \mathrm{e}^{-4 k r}
$$

for getting the Fourier transform but we proceed differently, as suggested by Helgason, p. 40-41. Namely, do the following. Write

$$
f(z)=f_{1}(r)=f_{2}(\tanh r)=F\left(\cosh ^{2} r\right),
$$

then calculate integrals

$$
\phi(u)=\int_{-\infty}^{\infty} F\left(u+y^{2}\right) \mathrm{d} y,
$$

set $\phi_{2}(u)=\phi\left(\cosh ^{2} u\right)$ and finally apply classical Fourier transform,

$$
\tilde{f}(\lambda)=\int_{-\infty}^{\infty} \mathrm{e}^{\mathrm{i} \lambda u} \phi_{2}(u) \mathrm{d} u=\sqrt{2 \pi} \hat{\phi}_{2}(\lambda),
$$

with the definition (5.15) we chose for the Fourier transform.

With our specific function we have $f_{1}(r)=\frac{4}{\mathrm{e}^{4 r}-1}$. Since $T=\tanh r=\frac{\mathrm{e}^{2 r}-1}{\mathrm{e}^{2 r}+1}$, we get $\mathrm{e}^{2 r}=\frac{1+T}{1-T}$, hence $\mathrm{e}^{4 r}=\frac{(1+T)^{2}}{(1-T)^{2}}$ and $f_{2}(T)=\frac{(1-T)^{2}}{T}$.

Next, for $q=\cosh ^{2} r$, we have $T^{2}=\frac{\left(\mathrm{e}^{r}+\mathrm{e}^{-r}\right)^{2}-4}{\left(\mathrm{e}^{r}+\mathrm{e}^{-r}\right)^{2}}=1-\frac{1}{q}$. Hence $T=\sqrt{(q-1) / q}$ and $F(q)=f_{2}(T)=$ $\sqrt{q /(q-1)}-2+\sqrt{(q-1) / q}$.

Furthermore,

$$
\begin{aligned}
\phi(u) & =\int_{-\infty}^{\infty} F\left(u+y^{2}\right) \mathrm{d} y=2 \int_{0}^{\infty}\left(\frac{\sqrt{u+y^{2}}}{\sqrt{u+y^{2}-1}}-2-\frac{\sqrt{u+y^{2}-1}}{\sqrt{u+y^{2}}}\right) \mathrm{d} y \\
& =2 \lim _{U \rightarrow \infty}\left(I_{1}(U)-2 U+I_{2}(U)\right)
\end{aligned}
$$


where

$$
\begin{aligned}
& I_{1}(U)=\int_{0}^{U} \frac{\sqrt{u+y^{2}}}{\sqrt{u+y^{2}-1}} \mathrm{~d} u:=\int_{0}^{U} \frac{\sqrt{a^{2}+y^{2}}}{\sqrt{b^{2}+y^{2}}} \mathrm{~d} u, \\
& I_{2}(U)=\int_{0}^{U} \frac{\sqrt{u+y^{2}-1}}{\sqrt{u+y^{2}}} \mathrm{~d} u:=\int_{0}^{U} \frac{\sqrt{b^{2}+y^{2}}}{\sqrt{a^{2}+y^{2}}} \mathrm{~d} u
\end{aligned}
$$

with $a^{2}=u>u-1=b^{2}$. Let $\alpha=\arctan (U / b)=\arctan (U /(u-1))$ and $q=\frac{b^{2}-a^{2}}{a}=\frac{1}{\sqrt{u}} \cdot$ According to formulas 3.169 and 8.111-8.112 in [15], we have

$$
\begin{aligned}
& I_{1}(U)=a[F(\alpha, q)-E(\alpha, q)]+U \frac{\sqrt{a^{2}+U^{2}}}{\sqrt{b^{2}+U^{2}}}, \\
& I_{2}(U)=\frac{b^{2}}{a} F(\alpha, q)-a E(\alpha, q)+U \frac{\sqrt{a^{2}+U^{2}}}{\sqrt{b^{2}+U^{2}}}
\end{aligned}
$$

where $F$ and $E$ are incomplete elliptic integrals of the first and of the second kind, respectively.

By summing up,

$$
I_{1}(U)-2 U+I_{2}(U)=\left(a+\frac{b^{2}}{a}\right) F(\alpha, q)-2 a E(\alpha, q)+2 U\left(\frac{\sqrt{a^{2}+U^{2}}}{\sqrt{b^{2}+U^{2}}}-1\right) .
$$

By taking a limit, note that the last term vanishes, and we obtain

$$
\begin{aligned}
\phi(u) & =2\left[\left(a+\frac{b^{2}}{a}\right) F(\pi / 2, q)-2 a E(\pi / 2, q)\right] \\
& =2\left[\left(a+\frac{b^{2}}{a}\right) \mathbb{F}(q)-2 a \mathbb{E}(q)\right] \\
& =2\left[\left(2 \sqrt{u}-\frac{1}{\sqrt{u}}\right) \mathbb{F}(q)-2 \sqrt{u} \mathbb{E}(q)\right] \\
& =4 \sqrt{u}\left(\mathbb{F}\left(\frac{1}{\sqrt{u}}\right)-\mathbb{E}\left(\frac{1}{\sqrt{u}}\right)\right)-\frac{2}{\sqrt{u}} \mathbb{F}\left(\frac{1}{\sqrt{u}}\right),
\end{aligned}
$$

where

$$
\mathbb{F}(q)=\int_{0}^{\pi / 2} \frac{\mathrm{d} \theta}{\sqrt{1-q^{2} \sin ^{2} \theta}}
$$

and

$$
\mathbb{E}(q)=\int_{0}^{\pi / 2} \sqrt{1-q^{2} \sin ^{2} \theta} \mathrm{d} \theta
$$

are complete elliptic integrals of the first and second kind, respectively.

We get a slightly clearer representation with

$$
\begin{aligned}
4 \sqrt{u}\left(\mathbb{F}\left(\frac{1}{\sqrt{u}}\right)-\mathbb{E}\left(\frac{1}{\sqrt{u}}\right)\right) & =\int_{0}^{\pi / 2} \frac{4 \sqrt{u}\left(1-\left(1-\sin ^{2} \theta / u\right)\right.}{\sqrt{1-\sin ^{2} \theta / u}} \mathrm{~d} \theta \\
& =4 \int_{0}^{\pi / 2} \frac{\sin ^{2} \theta}{\sqrt{u-\sin ^{2} \theta}} \mathrm{d} \theta .
\end{aligned}
$$


Hence

$$
\begin{gathered}
\phi(u)=2 \int_{0}^{\pi / 2} \frac{2 \sin ^{2} \theta-1}{\sqrt{u-\sin ^{2} \theta}} \mathrm{d} \theta, \\
\phi_{2}(u)=2 \int_{0}^{\pi / 2} \frac{2 \sin ^{2} \theta-1}{\sqrt{\cosh ^{2} u-\sin ^{2} \theta}} \mathrm{d} \theta .
\end{gathered}
$$

Recall that

$$
p(\lambda)=\frac{\hat{\phi}_{2}(\lambda) \lambda \tanh (\pi \lambda / 2)}{\sqrt{2 \pi}\left(\lambda^{2}+1\right)}
$$

and we are done.

\section{ORNSTEIN-UHLENBECK FIELDS}

We start with $\mathbb{R}^{1}$ and $\mathbb{R}^{n}$ analogies. A centered Gaussian one-parametric stationary process is called OU process if its covariance is $\mathbb{E} U(t) U(0)=\mathrm{e}^{-a|t|}$. Similarly (one version of) $\alpha$-fractional OU field is defined by $\mathbb{E} U(t) U(0)=\mathrm{e}^{-a|t|^{\alpha}}$. Here $0<\alpha \leq 2$. In Euclidean case time scaling is not a problem, so we set for a while $a=1$. The spectral measure for OU field is the Cauchy distribution $\nu(d u)=\frac{\mathrm{d} u}{\pi\left(1+u^{2}\right)} \cdot$ Analogously, for fractional OU field the spectral measure is the symmetric $\alpha$-stable distribution.

Next, consider OU stationary random field $U(t), t \in \mathbb{R}^{n}$, which we require to be OU field along any real line. It follows that the covariance should be $\mathbb{E} U(t) U(0)=\mathrm{e}^{-a|| t||}$. The corresponding spectral measure on $\mathbb{R}^{d}$ is the symmetric multivariate Cauchy distribution (for which $\mathrm{e}^{-a|| t||}$ is a characteristic function). There are two ways to define multivariate Cauchy distribution. One is to look at its Lévy-Khinchin formula. By scaling,

$$
\int_{S} \int_{0}^{\infty}\left(\mathrm{e}^{\mathrm{i}(t, r \theta)}-1\right) r^{-p} \mathrm{~d} r \mathrm{~d} \theta=c\|t\|^{p-1}, \quad 1<p<3 .
$$

Hence, $p=2$ provides a Lévy-Khinchin measure $r^{-2} d r \mathrm{~d} \theta$ for Cauchy distribution.

Another way to define Cauchy distribution goes through the connection of the density of a spherically symmetric distribution and the density of its one dimensional projection, see [39]. Indeed, let $\nu(\mathrm{d} x)=p\left(\|x\|^{2}\right) \mathrm{d} x$ and let $f$ be its marginal density. Then

$$
p(s)= \begin{cases}c \frac{\mathrm{d}^{m}}{\mathrm{~d} s^{m}} f(\sqrt{s}), & n=2 m+1 ; \\ c \frac{\mathrm{d}^{m}}{\mathrm{~d} s^{m}}\left[\int_{\sqrt{s}}^{\infty} \frac{z f(z) \mathrm{d} z}{\sqrt{z^{2}-s}}\right], & n=2 m .\end{cases}
$$

We know that marginal distribution is Cauchy. Hence $f(s)=\frac{c}{1+s^{2}}$ and we easily obtain $p(s)=c(1+s)^{-(n+1) / 2}$, hence,

$$
\nu(\mathrm{d} x)=c\left(1+\|x\|^{2}\right)^{-(n+1) / 2} \mathrm{~d} x .
$$

For further information about multivariate Cauchy distribution and some generalizations, we refer to [21] Chapter 42 and to [10].

We also obtain the spherical spectral measure:

$$
\begin{aligned}
\mathrm{e}^{-|| t||}=\int_{\mathbb{R}^{n}} \mathrm{e}^{\mathrm{i}(t, x)} \nu(\mathrm{d} x) & =c \int_{S} \int_{0}^{\infty} \mathrm{e}^{\mathrm{i}(t, x)} \frac{r^{n-1}}{\left(1+r^{2}\right)^{(n+1) / 2}} \mathrm{~d} r \mathrm{~d} \theta \\
& =c \int_{0}^{\infty} \varphi_{r}(t) \frac{r^{n-1}}{\left(1+r^{2}\right)^{(n+1) / 2}} \mathrm{~d} r
\end{aligned}
$$

Similarly, fractional OU random fields on $\mathbb{R}^{n}$ are defined by covariance $\mathbb{E} U(t) U(0)=\mathrm{e}^{-a|| t \mid \|^{\alpha}}$. The corresponding spectral measure is the symmetric $\alpha$-stable measure whose Lévy-Khinchin measure is given by 
$r^{-(\alpha+1)} \mathrm{d} r \mathrm{~d} \theta$ and there is no formula for its Lebesgue density. For $\alpha=2$ the spectral measure is standard Gaussian.

Remark: (a) The presented way is by far not the unique possibility to generalize the notion of OU process to fractional and/or to multivariate case. Even if time is one-dimensional, one can obtain another reasonable fractional extension of OU process by applying Lamperti transformation to the univariate fractional Brownian process $W^{H}$, that is considering $\widetilde{U}(t)=\mathrm{e}^{-H t} W^{H}\left(\mathrm{e}^{t}\right)$. The covariance of $\widetilde{U}$ is then

$$
\mathbb{E} \widetilde{U}(t) \widetilde{U}(0)=\frac{1}{2}\left(\mathrm{e}^{H t}+\mathrm{e}^{-H t}-\left|\mathrm{e}^{t / 2}-\mathrm{e}^{-t / 2}\right|^{2 H}\right) .
$$

(b) There is also a considerable literature about the extensions of OU process taking values in manifolds other than $\mathbb{R}^{n}$, see e.g. [36], focused on properties of OU as a diffusion. In spite of many common keywords, the latter approach apparently is completely disjoint with our constructions.

(c) OU processes on the line have important Markov property. The corresponding Markov concepts for random fields are too numerous and subtle to be discussed here. We refer to $[22,26,27,30]$ for related information about LBM and OU fields.

We now pass to fractional OU fields on hyperbolic space $\mathcal{L}_{n}$. As before, this should be a stationary random field with covariance $\mathbb{E} U(\eta) U(0)=\exp \left\{-a d_{n}(\eta, 0)^{\alpha}\right\}$ (in classical case, $\alpha=1$ ). In other words, $U$ would be a one-parametric fractional OU field along every geodesic line. Recall that parameter $a$ formally is now important, because we do not have appropriate time scaling in $\mathcal{L}_{n}$.

Recall that a function $\mathfrak{D}$ is negatively defined if and only if $\mathrm{e}^{-a \mathfrak{D}}$ is non-negatively defined for all $a>0$. Knowing that Lévy's fractional Brownian field exists for $H \leq 1 / 2$, we conclude that fractional OU is well defined for all $0<\alpha \leq 1, a>0$ and that it does not exist for $\alpha>1$ at least for all $a$ that are small enough. We do not know whether fractional OU does not exist for all $a>0$ in this case.

In the case of existence, it would be interesting to find the spherical spectral measure

$$
\mathrm{e}^{-a r^{\alpha}}=\int_{0}^{\infty} \omega_{s}(r) \nu_{a, \alpha}(\mathrm{d} s)
$$

When $\alpha<1$, for any $a>0$ the spectrum definitely is non-void in any neighborhood of zero, since covariance is decreasing slower than $\mathrm{e}^{-s r}$ for any particular $s$.

When $\alpha=1$, the spectrum does not approach 0 and its lower bound depends on parameter $a$. Indeed, decreasing as $\mathrm{e}^{-a r}, a<n-1 / 2$, prevents from the presence of spectrum in [0,a), cf. (4.27). On the other hand, if $a$ is large enough, $a>(n-1) / 2$, the covariance is in $L^{2}$ and hence only upper spectrum is present. Molchan in [30], Theorem 2, provides more detailed picture. In our notation, he gives a formula for the spectral density of OU-field with parameter $a$,

$$
g_{a}(\lambda)=\frac{a \Gamma\left(\frac{n+1}{2}\right)}{2^{n} \pi^{3 / 2} \Gamma(n / 2)}\left|\Gamma\left(\frac{n-1}{2}+\mathrm{i} \lambda\right)\right|^{2} \lambda \sinh (\pi \lambda) \frac{\left|\Gamma\left(a / 2-\frac{n-1}{4}+\mathrm{i} \lambda / 2\right)\right|^{2}}{\left|\Gamma\left(a / 2+\frac{n+3}{4}+\mathrm{i} \lambda / 2\right)\right|^{2}} .
$$

Notice that the $g_{a}$ vanishes at zero except for the values $a=\frac{n-1}{2}-2 j, j \in \mathbb{N}$ where $\Gamma$-function explodes in the nominator. We will see now that these values have also a special meaning for the lower spectrum.

Remark: We can not see what the complicated density $g_{a}$ has in common with Cauchy distribution from Euclidean case.

As for the lower spectrum, it is worthwhile to describe the evolution of the spectral measure of OU field when the parameter $a$ decreases from infinity to zero, as suggested by Theorem 2 in [30]. 
For OU field with covariance $a^{-1} \exp \{-a r\}$ the spectral weight is situated at the point $a+m \in\left(0, \frac{n-1}{2}\right)$, where $m$ is even integer, and the atom size is ([31])

$$
\begin{aligned}
w_{m}^{a}= & \frac{n-1-2 m-2 a}{4^{a}(n-1-m-2 a)} \frac{\Gamma(m+a)}{\Gamma(1+a) m !} \cdot \frac{\Gamma\left(1-\frac{n}{2}+a\right)}{\Gamma\left(1+\frac{m-n}{2}+a\right)} \cdot \frac{\Gamma\left(\frac{n+1}{2}\right)}{\Gamma\left(\frac{n+1}{2}-\frac{m}{2}\right)} \cdot \frac{\Gamma\left(\frac{1}{2}\right)}{\Gamma\left(\frac{1-m}{2}\right)} \\
& \times \frac{\Gamma(1+a)}{\Gamma\left(1+\frac{m}{2}+a\right)} \cdot \frac{\Gamma(n-1-m-a)}{\Gamma(n-1-m-2 a)} \cdot \frac{\Gamma\left(\frac{n}{2}-a\right)}{\Gamma\left(\frac{n}{2}\right)} .
\end{aligned}
$$

Therefore, the qualitative evolution picture is as follows. Let denote $\nu=\frac{n-1}{2}$ the crossing point of upper and lower spectra.

Once $a>\nu$, only the upper spectrum is present.

When $a=\nu$ an atom is born at $\nu$.

When $a$ decreases from $\nu$ to $\nu-2$, the atom floats through positions $a$.

When $a=\nu-2$ the second atom is born at $\nu$.

When $a$ decreases from $\nu-2$ to $\nu-4$, the first (resp. second) atom floats through positions $a$ (resp. $a+2$ ).

And so on: every time $a$ crosses a point $\nu-m$, with an even $m \in \mathbb{N}$, a new atom is born at $\nu$ and starts floating with unit speed towards zero.

Finally, when $a=0$ is achieved, we arrive to the spectrum of Lévy Brownian field. At this point the first atom arrives to 0 and this results in the appearance of a quadratic component. Other atoms will be situated in the even points of the open interval $(0, \nu)$ : namely, the atom born at $a=\nu-m$ will find its final place at $m$. This is in accordance with positions of atoms for LBF.

Of course, when comparing with LBF weights (8.4), we obtain $\lim _{a \rightarrow 0} w_{m}^{a}=w_{m}$, as $a \downarrow 0$.

On the other hand, notice that the baby atom (when it is just born) is very small: recall that the $m$ '-th atom appears at $a=\nu-m$, so that

$$
\lim _{a \rightarrow \nu-m}(n-1-2 m-2 a)=\lim _{a \rightarrow \nu-m}(n-1-2(m+a))=n-1-2 \nu=0 .
$$

Since $n-1-2 m-2 a$ is the first factor in the definition of $w_{m}^{a}$, we have

$$
\lim _{a \rightarrow \nu-m} w_{m}^{a}=0 .
$$

From the spectral measure of OU-fields associated to the covariance $\mathrm{e}^{-a u}$ one can deduce the spectral measure of a field with stationary increments and with structure functions $2\left(1-\mathrm{e}^{-a u}\right)$, as in (5.20). This enables us to obtain the spectral measure of many other fields. Indeed, let

$$
F(u)=\int_{0}^{\infty}\left(1-\mathrm{e}^{-a u}\right) \mu(\mathrm{d} a)
$$

be a Bernstein function. Then the spectral measure of a field with stationary increments and with structure function $F$ writes as

$$
\nu_{F}(\mathrm{~d} s)=\frac{1}{2} \int_{0}^{\infty} \nu_{a}(\mathrm{~d} s) \mu(\mathrm{d} a)
$$

where $\nu_{a}$ is the spectral measure of the OU-field just described above:

$$
\nu_{a}(\mathrm{~d} s)=g_{a}(\lambda) \mathrm{d} \lambda+a \sum_{\substack{m=0,2, \ldots \\ m+a \leq(n-1) / 2}} w_{m}^{a} \delta_{m+a} .
$$

For example, for $H$-fractional Lévy field we obtain

$$
\nu_{H}(\mathrm{~d} s)=\int_{0}^{\infty} \nu_{a}(\mathrm{~d} s) \frac{H \mathrm{~d} a}{\Gamma(1-2 H) a^{1+2 H}}, \quad 0<H<1 / 2 .
$$


Note that this spectral measure is absolutely continuous, both in upper and lower parts.

When $m=0$ and $a \rightarrow 0$, the atom $w_{0}^{a}$ located at the point $a \rightarrow 0$ has the asymptotics $w_{0}^{a} \sim \Gamma(a) \sim a^{-1}$. Therefore, the asymptotics of the spectral density at zero (lower spectrum) is $\frac{H}{\Gamma(1-2 H) a^{1+2 H}}$.

\section{QUADRATIC FIELD}

\subsection{Non-degeneracy of quadratic field}

Quadratic field on hyperbolic space is a field with stationary increments $\left\{X_{\eta}, \eta \in \mathcal{L}_{n}\right\}$ such that $\mathbb{E}\left|X_{\eta}\right|^{2}=$ $Q(\eta)$ where generalized quadratic form $Q$ is defined in (4.10) and plays a fundamental role in Lévy-Khinchin decomposition (5.21).

Notice that the Euclidean analogue of $Q$ is a simple quadratic form $Q_{\mathbb{R}}(x)=\|x\|^{2}$. Therefore, Euclidean quadratic field is just $X(t)=\sum_{1}^{n} t_{i} \xi_{i}, t \in \mathbb{R}^{n}$, with non-correlated r.v. $\xi_{i}$. This is a finite-rank field and its kernel is finite-dimensional.

In spite of this analogy, the quadratic field on hyperbolic space is not as degenerate. Just take $n=2$ and consider the behavior of $X$ along a fixed geodesic line. This restriction produces a 1-parametric process with stationary increments (still denote it $X$ ) such that, according to (4.12),

$$
\mathbb{E}\left|X_{r}\right|^{2}=Q(\eta)=2 \log \cosh (r / 2), \quad r=d(\eta, 0) \geq 0 .
$$

We are interested in the derivative $X^{\prime}$ of $X$. Recall that for any differentiable process $Y$ with stationary increments the derivative is a stationary process with covariance

$$
\mathbb{E} Y_{t}^{\prime} Y_{0}^{\prime}=\lim _{\delta \rightarrow 0} \delta^{-2} \mathbb{E}\left(Y_{t+\delta}-Y_{t}\right) Y_{\delta}=\frac{1}{2} \lim _{\delta \rightarrow 0} \delta^{-2}\left[\mathbb{E}\left|Y_{t+\delta}\right|^{2}+\mathbb{E}\left|Y_{t-\delta}\right|^{2}-2 \mathbb{E}\left|Y_{t}\right|^{2}\right]=\left.\frac{1}{2} \frac{\mathrm{d}^{2}}{\mathrm{~d} t^{2}}\right|_{t} \mathbb{E}\left|Y_{t}\right|^{2} .
$$

In our case, $\mathbb{E} X_{r}^{\prime} X_{0}^{\prime}=\frac{1}{2} Q^{\prime \prime}(r)=\frac{1}{4} \cosh ^{-2}\left(\frac{r}{2}\right)$. We are even able to find the spectral measure of $X^{\prime}$. Namely, according to Formula 3.982 in [15],

$$
\int_{0}^{\infty} \frac{\cos a r}{\cosh ^{2} \beta r} \mathrm{~d} r=\frac{a \pi}{2 \beta^{2} \sinh (a \pi / 2 \beta)}, \quad a>0, \operatorname{Re} \beta>0,
$$

hence the spectral density for $X^{\prime}$ is

$$
f(a)=\frac{1}{2 \pi} \int_{-\infty}^{\infty} \frac{\mathrm{e}^{\mathrm{i} a r}}{4 \cosh ^{2}(r / 2)} \mathrm{d} r=\frac{a}{2 \sinh (a \pi)} .
$$

We see that $X^{\prime}$ is an infinitely differentiable (since the spectral measure has all moments) and infinite-rank process. Hence, the same is true for $X$ itself.

\subsection{Helix representation}

In this subsection we prove that a helix given in [11] provides a covariance structure of quadratic process. Our starting point will be a stationary field $X_{s}$ with the covariance (5.7) associated to the spherical function $\omega_{s}$. Define a field with stationary increments $Y_{s}(\eta)=s^{-1 / 2}\left(X_{s}(\eta)-X_{s}(\overline{0})\right.$. Then $\operatorname{Cov}\left(Y_{s}\left(\eta_{1}\right), Y_{s}\left(\eta_{2}\right)\right)$ equals to

$$
s^{-1} \gamma(s, n)^{-1} \int_{\bar{B}} \int_{\bar{B}}\left(\left[\eta_{1}, \bar{b}_{1}\right]^{-(n-1-s)}-1\right)\left(\left[\eta_{2}, \bar{b}_{2}\right]^{-(n-1-s)}-1\right)\left[\bar{b}_{1}, \bar{b}_{2}\right]^{-s} \mathrm{~d} \bar{b}_{1} \mathrm{~d} \bar{b}_{2} .
$$

In particular,

$$
\begin{aligned}
\mathbb{E}\left|Y_{s}(\eta)\right|^{2} & =2 s^{-1}\left(1-\omega_{s}(\eta)\right) \\
& =s^{-1} \gamma(s, n)^{-1} \int_{\bar{B}} \int_{\bar{B}}\left(\left[\eta, \bar{b}_{1}\right]^{-(n-1-s)}-1\right)\left(\left[\eta, \bar{b}_{2}\right]^{-(n-1-s)}-1\right)\left[\bar{b}_{1}, \bar{b}_{2}\right]^{-s} \mathrm{~d} \bar{b}_{1} \mathrm{~d} \bar{b}_{2} .
\end{aligned}
$$


Notice also that

$$
\begin{aligned}
& \int_{\bar{B}} \int_{\bar{B}}\left(\left[\eta, \bar{b}_{1}\right]^{-(n-1-s)}-1\right)\left(\left[\eta, \bar{b}_{2}\right]^{-(n-1-s)}-1\right) \mathrm{d} \bar{b}_{1} \mathrm{~d} \bar{b}_{2}=\int_{\bar{B}}\left(\left[\eta, \bar{b}_{1}\right]^{-(n-1-s)}-1\right) \mathrm{d} \bar{b}_{1} \\
& \times \int_{\bar{B}}\left(\left[\eta, \bar{b}_{2}\right]^{-(n-1-s)}-1\right) \mathrm{d} \bar{b}_{2}=\left(\omega_{n-1-s}(\eta)-1\right)^{2}=\left(\omega_{s}(\eta)-1\right)^{2}=O\left(s^{2}\right), \quad s \rightarrow 0 .
\end{aligned}
$$

We see that

$$
\begin{aligned}
Q(\eta) & =\lim _{s \rightarrow 0} s^{-1}\left(1-\omega_{s}(\eta)\right)=\lim _{s \rightarrow 0} \frac{1}{2} \mathbb{E}\left|Y_{s}(\eta)\right|^{2} \\
& =\lim _{s \rightarrow 0} s^{-1}(2 \gamma(s, n))^{-1} \int_{\bar{B}} \int_{\bar{B}}\left(\left[\eta, \bar{b}_{1}\right]^{-(n-1-s)}-1\right)\left(\left[\eta, \bar{b}_{2}\right]^{-(n-1-s)}-1\right)\left(\left[\bar{b}_{1}, \bar{b}_{2}\right]^{-s}-1\right) \mathrm{d} \bar{b}_{1} \mathrm{~d} \bar{b}_{2} \\
& =-(2 \gamma(0, n))^{-1} \int_{\bar{B}} \int_{\bar{B}}\left(\left[\eta, \bar{b}_{1}\right]^{-(n-1)}-1\right)\left(\left[\eta, \bar{b}_{2}\right]^{-(n-1)}-1\right) \log \left(\left[\bar{b}_{1}, \bar{b}_{2}\right]\right) \mathrm{d} \bar{b}_{1} \mathrm{~d} \bar{b}_{2} .
\end{aligned}
$$

Therefore, the helix of functions $\left(F_{\eta}: \bar{B} \rightarrow \mathbb{R}\right)_{\eta \in \mathcal{L}_{n}}$ given by

$$
F_{\eta}(\bar{b})=(2 \gamma(0, n))^{-1 / 2}\left([\eta, \bar{b}]^{-(n-1)}-1\right)
$$

considered in the Hilbert space with scalar product

$$
\left(f_{1}, f_{2}\right)=-\int_{\bar{B}} \int_{\bar{B}} f_{1}\left(\bar{b}_{1}\right) \overline{f_{2}\left(\bar{b}_{2}\right)} \log \left(\left[\bar{b}_{1}, \bar{b}_{2}\right]\right) \mathrm{d} \bar{b}_{1} \mathrm{~d} \bar{b}_{2}
$$

has the same Hilbert structure as a quadratic process.

\subsection{Integral representation}

Again we use that a quadratic field $X^{Q}(\eta)$ can be obtained as the limit of $(2 s)^{-1 / 2}\left(X_{s}(\eta)-X_{s}(\overline{0})\right.$ as $s \rightarrow 0$. By using the white noise representation for $X_{s}$ given in (5.11) we obtain

$$
X^{Q}(\eta)=\int_{\mathbb{R}^{n-1}} m_{\eta}^{Q}(x) W(\mathrm{~d} x)
$$

where

$$
m_{\eta}^{Q}(x)=\lim _{s \rightarrow 0}(2 s)^{-1 / 2}\left(m_{\eta}(x)-m_{\overline{0}}(x)\right)=\lim _{s \rightarrow 0}(2 s)^{-1 / 2}\left(\frac{Z(s, n)}{2 \gamma(s, n)}\right)^{1 / 2} I_{s}(x),
$$

with $I_{s}(x):=\left(\frac{Z(s, n)}{2 \gamma(s, n)}\right)^{-1 / 2}\left(m_{\eta}(x)-m_{\overline{0}}(x)\right)$. Moreover,

$$
\begin{aligned}
\lim _{s \rightarrow 0}(2 s)^{-1 / 2}\left(\frac{Z(s, n)}{2 \gamma(s, n)}\right)^{1 / 2} & =\lim _{s \rightarrow 0}(2 s)^{-1 / 2}\left(\frac{\Gamma\left(\frac{n-1}{4}+\frac{s}{2}\right)^{2} \Gamma\left(\frac{n-1}{2}-s\right)}{\Gamma\left(\frac{n-1}{4}-\frac{s}{2}\right)^{2} \Gamma(s) \pi^{\frac{n-1}{2}}} \cdot \frac{\pi^{1 / 2} \Gamma(n-1-s)}{\Gamma(n / 2) \Gamma\left(\frac{n-1}{2}\right) 2^{n-s}}\right)^{1 / 2} \\
& =\left(\frac{\Gamma(n-1)}{\Gamma(n / 2) 2^{n+1} \pi^{\frac{n}{2}-1}}\right)^{1 / 2} \lim _{s \rightarrow 0}(s \Gamma(s))^{-1 / 2} \\
& =\left(\frac{\Gamma(n-1)}{\Gamma(n / 2) 2^{n+1} \pi^{\frac{n}{2}-1}}\right)^{1 / 2}
\end{aligned}
$$


and

$$
\begin{aligned}
\lim _{s \rightarrow 0} I_{s}(x) & =\lim _{s \rightarrow 0} \int_{\mathbb{R}^{n-1}}\left\|x-x_{1}\right\|^{\frac{1-n}{2}-s} J_{\mathcal{P}}\left(x_{1}\right)^{1 / q}\left(\left[\eta, \mathcal{P}\left(x_{1}\right)\right]^{-(n-1-s)}-1\right) \mathrm{d} x_{1} \\
& =\int_{\mathbb{R}^{n-1}}\left\|x-x_{1}\right\|^{\frac{1-n}{2}} J_{\mathcal{P}}\left(x_{1}\right)\left(\left[\eta, \mathcal{P}\left(x_{1}\right)\right]^{-(n-1)}-1\right) \mathrm{d} x_{1} .
\end{aligned}
$$

Therefore,

$$
m_{\eta}^{Q}(x)=\left(\frac{\Gamma(n-1)}{\Gamma(n / 2) 2^{n+1} \pi^{\frac{n}{2}-1}}\right)^{1 / 2} \int_{\mathbb{R}^{n-1}}\left\|x-x_{1}\right\|^{\frac{1-n}{2}} J_{\mathcal{P}}\left(x_{1}\right)\left(\left[\eta, \mathcal{P}\left(x_{1}\right)\right]^{-(n-1)}-1\right) \mathrm{d} x_{1} .
$$

For $n=2$, we obtain

$$
m_{\eta}^{Q}(x)=2^{-3 / 2} \int_{\mathbb{R}^{1}}\left\|x-x_{1}\right\|^{-\frac{1}{2}} J_{\mathcal{P}}\left(x_{1}\right)\left(\left[\eta, \mathcal{P}\left(x_{1}\right)\right]^{-1}-1\right) \mathrm{d} x_{1} .
$$

When we use the Poincaré half plane we get for the integral representation with respect to the Fourier white noise for $\zeta \in \mathbb{P}$

$$
\begin{aligned}
\widehat{m}_{\zeta}^{Q}(u) & =(2|u|)^{-1 / 2}\left(\left(\Im(\zeta)(\Re \zeta-t)^{2}+\widehat{\Im(\zeta)^{2}}\right)^{-1}-\left(1+t^{2}\right)^{-1}\right)(u) . \\
& =\pi(2|u|)^{-1 / 2}\left(\mathrm{e}^{-2 \pi(i \Re \zeta u+\Im \zeta|u|)}-\mathrm{e}^{-2 \pi|u|}\right) .
\end{aligned}
$$

One can remark that the quadratic field is not generated by finite dimensional white noise in contrast with the Euclidean case.

\section{WhITE NOISE}

In this section, we let $n=2$. Since the white noise is a generalized process, it is defined on test functions. Following the analogy with Euclidean case, it is natural to define a white noise on $\mathbb{D}$ as a field $\mathcal{W}[f], f \in L^{2}(\mathbb{D}, d z)$, with covariance

$$
\operatorname{Cov}\left(\mathcal{W}\left[f_{1}\right], \mathcal{W}\left[f_{2}\right]\right)=\int_{\mathbb{D}} f_{1}(z) \overline{f_{2}(z)} \mathrm{d} z
$$

By using Fourier transform, we can also write this as

$$
\operatorname{Cov}\left(\mathcal{W}\left[f_{1}\right], \mathcal{W}\left[f_{2}\right]\right)=\int_{0}^{\infty} \int_{B} \tilde{f}_{1}(\lambda, b) \overline{\tilde{f}_{2}(\lambda, b)} \mathrm{d} b \frac{\lambda \tanh (\pi \lambda / 2) \mathrm{d} \lambda}{2 \pi}
$$

Let us discuss the spectral measure of white noise. For any stationary field $X_{z}$ on $\mathbb{D}$ with a spectral measure $\nu$ supported on the upper spectrum (see (5.3)) let $X[f]=\int_{\mathbb{D}} f(z) X(z) d z$. We have

$$
\operatorname{Cov}\left(X\left[f_{1}\right], X\left[f_{2}\right]\right)=\int_{\mathbb{D}} \int_{\mathbb{D}} f_{1}\left(z_{1}\right) \overline{f_{2}\left(z_{2}\right)} \operatorname{Cov}\left(X_{z_{1}}, X_{z_{2}}\right) \mathrm{d} z_{1} \mathrm{~d} z_{2} .
$$

By spectral representation

$$
\operatorname{Cov}\left(X_{z_{1}}, X_{z_{2}}\right)=\int_{0}^{\infty} K_{\lambda}\left(z_{1}, z_{2}\right) \nu(\mathrm{d} \lambda)=\int_{0}^{\infty} \int_{B} \mathrm{e}^{(1+\mathrm{i} \lambda)\left\langle z_{1}, b\right\rangle+(1-\mathrm{i} \lambda)\left\langle z_{2}, b\right\rangle} \mathrm{d} b \nu(\mathrm{d} \lambda)
$$


where $K_{\lambda}$ is the covariance from (5.2). It follows that

$$
\begin{aligned}
\operatorname{Cov}\left(X\left[f_{1}\right], X\left[f_{2}\right]\right) & =\int_{\mathbb{D}} \int_{D} f_{1}(z) \bar{f}_{2}(z) \int_{0}^{\infty} \int_{B} \mathrm{e}^{(1+\mathrm{i} \lambda)\left\langle z_{1}, b\right\rangle+(1-i \lambda)\left\langle z_{2}, b\right\rangle} \mathrm{d} b \nu(\mathrm{d} \lambda) \\
& =\int_{0}^{\infty} \int_{B} \int_{\mathbb{D}} f_{1}\left(z_{1}\right) \mathrm{e}^{(1+\mathrm{i} \lambda)\left\langle z_{1}, b\right\rangle} \mathrm{d} z_{1} \cdot \int_{\mathbb{D}} \bar{f}_{2}\left(z_{2}\right) \mathrm{e}^{(1-\mathrm{i} \lambda)\left\langle z_{2}, b\right\rangle} \mathrm{d} z_{2} \mathrm{~d} b \nu(\mathrm{d} \lambda) \\
& =\int_{0}^{\infty} \int_{B} \tilde{f}_{1}(\lambda, b) \overline{\tilde{f}_{2}(\lambda, b)} \mathrm{d} b \nu(\mathrm{d} \lambda) .
\end{aligned}
$$

In the case of white noise, by comparing this with the formula above we conclude that

$$
\nu_{\mathcal{W}}(\mathrm{d} \lambda)=\frac{\lambda \tanh (\pi \lambda / 2) \mathrm{d} \lambda}{2 \pi}
$$

\section{EuCLIDEAN SPHERE}

In this section we provide a brief survey of the same problems considered on the unit Euclidean sphere $\mathbb{S}=\mathbb{S}^{n} \subset \mathbb{R}^{n+1}$ instead of hyperbolic space. Compactness of $\mathbb{S}$ simplifies the problems considerably.

\section{1. $\mathbb{S}$ as a symmetric space}

We can consider $\mathbb{S}$ as a symmetric space equal to the factor of all rotations $S O(n+1)$ of $\mathbb{S}$ factorized by the set of rotations $S O(n)$ preserving one given point (which is equivalent to all rotations of $\mathbb{S}^{n-1}$ ). In the following we let $\overline{0}=(1,0, \ldots, 0)$ be a fixed point.

The space $\mathbb{S}$ is a Riemann manifold with the distance $d(\cdot, \cdot) \in[0, \pi]$ given by

$$
\cos d\left(\eta, \eta^{\prime}\right)=\left(\eta, \eta^{\prime}\right)_{\mathbb{R}^{n+1}} .
$$

The invariant measure is the surface measure of the sphere. We normalize it so that the total mass would be one.

The geodesic lines are large circles. Thus the shortest way between two points goes along a large circle. There are infinitely many such ways. In particular, if the points are opposite poles, then there are infinitely many shortest ways (meridians) connecting the points.

The volume of an $r$-ball in $\mathbb{S}^{n}$ is

$$
V(r)=\left|\mathbb{S}^{n-1}\right| \int_{0}^{r} \sin ^{n-1} \theta \mathrm{d} \theta=\frac{2 \pi^{n / 2}}{\Gamma(n / 2)} \int_{0}^{r} \sin ^{n-1} \theta \mathrm{d} \theta .
$$

Hence, the surface measure $A$ of the $r$-ball on the sphere is defined by

$$
A(r)=\frac{\mathrm{d}}{\mathrm{d} r} V(r)=\frac{2 \pi^{n / 2}}{\Gamma(n / 2)} \sin ^{n-1} r .
$$

The Laplace-Beltrami operator on $\mathbb{S}$ can be defined as [11], p. 200,

$$
\Delta f=\frac{\mathrm{d}^{2}}{\mathrm{~d} r^{2}} f+\frac{A^{\prime}(r)}{A(r)} \frac{\mathrm{d}}{\mathrm{d} r} f=\frac{\mathrm{d}^{2}}{\mathrm{~d} r^{2}} f+(n-1) \cot r \frac{\mathrm{d}}{\mathrm{d} r} f .
$$

The spherical functions are [11], p. 203.

$$
\omega_{s}(\eta)=\int_{\mathbb{S}^{n-1}}\left[(\overline{0}, \eta)_{\mathbb{R}^{n+1}}-i(b, \eta)_{\mathbb{R}^{n+1}}\right]^{s} \mathrm{~d} b, \quad s=0,1,2, \ldots,
$$


where $\mathbb{S}^{n-1}=\left\{b \in \mathbb{S}: b_{0}=0\right\}$. In coordinate form

$$
\omega_{s}(\eta)=\frac{\Gamma(n / 2)}{\sqrt{\pi} \Gamma\left(\frac{n-1}{2}\right)} \int_{0}^{\pi}[\cos r-i \sin r \cos \theta]^{s} \sin ^{n-2} \theta \mathrm{d} \theta=P_{n, s}(\cos r) .
$$

Here (and elsewhere in this section) $r=d(\eta, \overline{0})$ and $P_{n, s}$ are ultra-spherical polynomials (Gegenbauer polynomials).

Examples: $P_{n, 0}(u)=1, P_{n, 1}(u)=u, P_{n, 2}=\frac{(n+1) u^{2}-1}{n}, P_{n, 3}=\frac{(n+3) u^{3}-3 u}{n}$.

The spherical functions satisfy (see [11], p. 203)

$$
\Delta \omega_{s}=-s(s+n-1) \omega_{s} .
$$

\subsection{Lévy-Khinchin formulas}

The non-negative type functions (covariance functions of spherically invariant stationary fields) have the form $K(x)=K(d(x, \overline{0}))$,

$$
K(r)=\sum_{s=0}^{\infty} \nu_{s} \omega_{s}(x)=\sum_{s=0}^{\infty} \nu_{s} P_{n, s}(\cos r),
$$

with spectrum $\left(\nu_{j}\right)$ satisfying $\nu_{j} \geq 0, \sum_{s=0}^{\infty} \nu_{s}<\infty$.

Moreover, the functions of negative type (structure functions of the spherically invariant fields with stationary increments), have the form $\mathfrak{D}(x)=\mathfrak{D}(r)$,

$$
\mathfrak{D}(r)=\sum_{s=0}^{\infty} \nu_{s}\left(1-\omega_{s}(x)\right)=\sum_{s=0}^{\infty} \nu_{s}\left(1-P_{n, s}(\cos r)\right),
$$

with the same assumptions on $\left(\nu_{j}\right)$.

We see that every field with stationary increments on the sphere is generated by a stationary process. There is no generalized quadratic form and there is no possibility for infinite spectral measure since there is no spectrum in the neighborhood of zero.

\subsection{Examples of stationary fields on Euclidean sphere}

Example 12.1. Let $\left(\xi_{j}\right)_{0 \leq j \leq n}$ be centered i.i.d. with unit variance. Let $X(\eta)=\sum_{j=0}^{n} \eta_{j} \xi_{j}$ for $\eta \in \mathbb{S}^{n}$. Then $\operatorname{cov}\left(X(\eta), X\left(\eta^{\prime}\right)\right)=\left(\eta, \eta^{\prime}\right)_{\mathbb{R}^{n+1}}=\cos d\left(\eta, \eta^{\prime}\right)$. We see that the degenerated field $X$ corresponds to the spherical function $\omega_{n, 1}$.

Example 12.2. Let $\left(\xi_{j_{1} j_{2}}\right)_{0 \leq j_{1}, j_{2} \leq n}$ be centered i.i.d. with unit variance. Let $X(\eta)=\sum_{j_{1}, j_{2}=0}^{n} \eta_{j_{1}} \eta_{j_{2}} \xi_{j_{1} j_{2}}$ for $\eta \in \mathbb{S}^{n}$. Then

$$
\operatorname{cov}\left(X(\eta), X\left(\eta^{\prime}\right)\right)=\sum_{j_{1}, j_{2}} \eta_{j_{1}} \eta_{j_{2}} \eta_{j_{1}}^{\prime} \eta_{j_{2}}^{\prime}=\left(\sum_{j} \eta_{j} \eta_{j}^{\prime}\right)^{2}=\left(\eta, \eta^{\prime}\right)_{\mathbb{R}^{n+1}}^{2}=\cos ^{2} d\left(\eta, \eta^{\prime}\right)
$$

Similarly, $k$-linear forms with independent coefficients generate fields with covariance $\cos ^{k} d\left(\eta, \eta^{\prime}\right)$.

Example 12.3. Let $X$ be a field from the previous example. We center it by subtracting its integral:

$$
I:=\int_{\mathbb{S}} X(\eta) \mathrm{d} \eta=(n+1)^{-1} \sum_{j=0}^{n} \xi_{j j}
$$


Let $X_{0}(\eta)=X(\eta)-I$. The covariance of the new field is

$$
\operatorname{cov}\left(X_{0}(\eta), X_{0}\left(\eta^{\prime}\right)\right)=\operatorname{cov}\left(X(\eta), X\left(\eta^{\prime}\right)\right)-\operatorname{cov}(X(\eta), I)-\operatorname{cov}\left(X\left(\eta^{\prime}\right), I\right)+\operatorname{cov}(I, I) .
$$

Clearly,

$$
\operatorname{cov}(X(\eta), I)=(n+1)^{-1} \operatorname{cov}\left(\sum_{j=0}^{n} \eta_{j_{1}} \eta_{j_{2}} \xi_{j_{1} j_{2}}, \sum_{j=0}^{n} \xi_{j j}\right)=(n+1)^{-1} \sum_{j=0}^{n} \eta_{j}^{2}=(n+1)^{-1},
$$

and by the same reason, $\operatorname{cov}\left(X\left(\eta^{\prime}\right), I\right)=(n+1)^{-1}$. Next,

$$
\operatorname{cov}(I, I)=(n+1)^{-2} \sum_{j_{1}=0}^{n} \sum_{j_{2}=0}^{n} \operatorname{cov}\left(\xi_{j_{1} j_{1}}, \xi_{j_{2} j_{2}}\right)=(n+1)^{-1} .
$$

It follows that

$$
\operatorname{cov}\left(X_{0}(\eta), X_{0}\left(\eta^{\prime}\right)\right)=\cos ^{2} d\left(\eta, \eta^{\prime}\right)-(n+1)^{-1}=\frac{n}{n+1} P_{n, 2}\left(\cos d\left(\eta, \eta^{\prime}\right)\right) .
$$

We see that the field $X_{0}$ corresponds to the second non-trivial spherical function (up to a constant).

We briefly sketch here the next step. Take the third order field from the previous example,

$$
X(\eta)=\sum_{j_{1}, j_{2}, j_{3}=0}^{n} \eta_{j_{1}} \eta_{j_{2}} \eta_{j_{3}} \xi_{j_{1} j_{2} j_{3}}
$$

It is easy to see that $\int_{\mathbb{S}} X(\eta) \mathrm{d} \eta=0$, thus the centering will be more involved. Namely, we center $X$ so that it becomes orthogonal in $L^{2}(\mathbb{S})$ not only to constants but also to all linear functions. To this aim, let

$$
\begin{aligned}
X_{0}(\eta) & =X(\eta)-\sum_{l=0}^{n} \frac{\int_{\mathbb{S}} X(u) u_{l} \mathrm{~d} u}{\int_{\mathbb{S}} u_{l}^{2} \mathrm{~d} u} \eta_{l} \\
& =X(\eta)-\sum_{l=0}^{n} \sum_{j \neq l}\left(\xi_{j j l}+\xi_{j l j}+\xi_{l j j}\right) \frac{\eta_{l}}{n+3}-\sum_{l=0}^{n} \xi_{l l l} \frac{3 \eta_{l}}{n+3} .
\end{aligned}
$$

Here we used that

$$
\begin{aligned}
\int_{\mathbb{S}} X(u) u_{l} d u & =\sum_{j_{1}, j_{2}, j_{3}=0}^{n} \xi_{j_{1} j_{2} j_{3}} \int_{\mathbb{S}} u_{j_{1}} u_{j_{2}} u_{j_{3}} u_{l} \mathrm{~d} u \\
& =\sum_{j \neq l}\left(\xi_{j j l}+\xi_{j l j}+\xi_{l j j}\right) \int_{\mathbb{S}} u_{j}^{2} u_{l}^{2} \mathrm{~d} u+\xi_{l l l} \int_{\mathbb{S}} u_{l}^{4} \mathrm{~d} u \\
& =\sum_{j \neq l}\left(\xi_{j j l}+\xi_{j l j}+\xi_{l j j}\right) \frac{1}{(n+1)(n+3)}+\xi_{l l l} \frac{3}{(n+1)(n+3)} .
\end{aligned}
$$

An easy direct calculation shows that

$$
\operatorname{cov}\left(X_{0}(\eta), X_{0}\left(\eta^{\prime}\right)\right)=\cos ^{3} d\left(\eta, \eta^{\prime}\right)-\frac{3 \cos d\left(\eta, \eta^{\prime}\right)}{n+3}=\frac{n}{n+3} P_{n, 3}\left(\cos d\left(\eta, \eta^{\prime}\right)\right) .
$$

Again we see that the field $X_{0}$ corresponds to a non-trivial spherical function (up to a constant).

It is very likely that the fields corresponding to other spherical functions can be obtained from the fields of Example 12.2 by similar centering (projection operation). This procedure is strikingly similar to the normalization procedure for $U$-statistics. We stress that all basic stationary fields on $\mathbb{S}$ are finite-rank fields. 
Example 12.4. (Lévy's Brownian field on the sphere). For $\eta \in \mathbb{S}$ let $A_{\eta}=\left\{b \in \mathbb{S}:(b, \eta)_{\mathbb{R}^{n+1}}>0\right\}$. Take a white noise $W$ on $\mathbb{S}$ and consider the field $X(\eta)=W\left(A_{\eta}\right)$. Then $\operatorname{cov}\left(X(\eta), X\left(\eta^{\prime}\right)\right)=\left|A_{\eta} \cap A_{\eta^{\prime}}\right|$. Let us calculate this measure. Clearly, it only depends on the distance $r=d\left(\eta, \eta^{\prime}\right)$ (hence, $X$ is a stationary process). Let for simplicity $\eta=(1,0, \ldots, 0), \eta^{\prime}=(\cos r, \sin r, 0, \ldots, 0)$. Then

$$
A_{\eta} \cap A_{\eta^{\prime}}=\left\{u \in \mathbb{S}: u_{0}=\rho \cos \psi, u_{1}=\rho \sin \psi, 0 \leq \rho \leq 1, r-\pi / 2 \leq \psi \leq \pi / 2\right\} .
$$

By rotation symmetry of $\mathbb{S}$,

$$
\left|A_{\eta} \cap A_{\eta^{\prime}}\right|=\pi / 2-(r-\pi / 2)=\pi-r=\pi-d\left(\eta, \eta^{\prime}\right) .
$$

It follows that

$$
\frac{1}{2} \mathbb{E}(X(\eta)-X(\overline{0}))^{2}=\mathbb{E} X(\overline{0})^{2}-\mathbb{E} X(\eta) X\left(\eta^{\prime}\right)=\pi-\left(\pi-d\left(\eta, \eta^{\prime}\right)\right)=d\left(\eta, \eta^{\prime}\right) .
$$

We see that the field with stationary increments $2^{-1 / 2}(X(\eta)-X(\overline{0}))$ is a Lévy's Brownian field.

\subsection{Illustration: $n=1$}

The theory for $\mathbb{S}^{1}$ is particularly simple because it reduces to the well known case of periodic stationary processes. We identify $\mathbb{S}^{1}$ with $[0,2 \pi)$. Covariance function of any complex $2 \pi$-periodical stationary process writes as

$$
K(\theta)=\sum_{s \in \mathbb{Z}} \nu_{s} \mathrm{e}^{\mathrm{i} s \theta}
$$

with summable and positive spectral weights $\left(\nu_{s}\right)$, which can be calculated as

$$
\nu_{s}=\frac{1}{2 \pi} \int_{0}^{2 \pi} K(\theta) \mathrm{e}^{-\mathrm{i} s \theta} \mathrm{d} \theta
$$

once $K$ is given. For real fields $\nu_{s}=\nu_{-s}$ and we have

$$
K(\theta)=\sum_{s \geq 0} \tilde{\nu}_{s} \cos (s \theta)
$$

Here the functions $\omega_{s}(\theta)=\cos (s \theta)$ are the spherical functions corresponding to eigenvalues $-s^{2}=-s(s+n-1)$ and $\tilde{\nu}_{0}=\nu_{0} ; \tilde{\nu}_{s}=2 \nu_{s}($ for $s>0)$.

All stationary processes admit a simple integral representation. Let $f \in L^{2}[0,2 \pi)$. We set

$$
X(\eta)=\int_{0}^{2 \pi} f(\theta+\eta) W(\mathrm{~d} \theta)
$$

where $W$ is a white noise controlled by Lebesgue measure. Let us calculate the covariance $K_{X}$ by using Fourier expansion

$$
f(\theta)=\sum_{s \in \mathbb{Z}} \hat{f}_{s} \mathrm{e}^{\mathrm{i} s \theta}
$$

Since

$$
f(\theta+\eta)=\sum_{s \in \mathbb{Z}} \hat{f}_{s} \mathrm{e}^{\mathrm{i} s \eta} \mathrm{e}^{\mathrm{i} s \theta}
$$


we obtain by Parseval equality

$$
\begin{aligned}
K_{X}\left(\eta_{1}, \eta_{2}\right) & =\int_{0}^{2 \pi} f\left(\theta+\eta_{1}\right) \overline{f\left(\theta+\eta_{2}\right)} \mathrm{d} \theta \\
& =2 \pi \sum_{s \in \mathbb{Z}} \hat{f}_{s} \mathrm{e}^{\mathrm{i} s \eta_{1}} \overline{\hat{f}_{s} \mathrm{e}^{\mathrm{i} s \eta_{2}}}=2 \pi \sum_{s \in \mathbb{Z}}\left|\hat{f}_{s}\right|^{2} \mathrm{e}^{\mathrm{i} s\left(\eta_{1}-\eta_{2}\right)} .
\end{aligned}
$$

We see that $\nu_{s}=2 \pi\left|\hat{f}_{s}\right|^{2}$, hence any spectrum can be obtained within this family of fields.

The fields with stationary increments are all generated by stationary fields. They admit an integral representation

$$
Y(\eta)=X(\eta)-X(0)=\int_{0}^{2 \pi}(f(\theta+\eta)-f(\theta)) W(\mathrm{~d} \theta) .
$$

As usual, $\mathfrak{D}_{Y}(\eta)=2\left(K_{X}(0)-K_{X}(\eta)\right)$. Notice that adding a constant to $X$ does not change $Y$. Hence the spectrum of $X$ at zero is irrelevant for $Y$.

Consider now a particular case of fractional Brownian process $W^{H}, 0<H<1$, and find the respective stationary field $X_{H}$ with covariance $K_{H}$ and spectrum $\left(\nu_{s, H}\right)_{s \in \mathbb{Z}}$. We have

$$
K_{H}(\eta)=K_{H}(0)-\frac{\mathfrak{D}_{H}(\eta)}{2}=K_{H}(0)-\frac{\mathrm{d}(\eta, 0)}{2}=K_{H}(0)-\frac{\min \{|\eta|,|2 \pi-\eta|\}^{2 H}}{2} .
$$

For $s \neq 0$, by integrating we find

$$
\begin{aligned}
\nu_{s, H} & =-\frac{1}{4 \pi} \int_{0}^{2 \pi} \min \{|\eta|,|2 \pi-\eta|\}^{2 H} \mathrm{e}^{-\mathrm{i} s \eta} \mathrm{d} \eta=-\frac{1}{4 \pi} \int_{-\pi}^{\pi}|\eta|^{2 H} \mathrm{e}^{-\mathrm{i} s \eta} \mathrm{d} \eta \\
& =-\frac{1}{2 \pi} \int_{0}^{\pi} \eta^{2 H} \cos (s \eta) \mathrm{d} \eta
\end{aligned}
$$

One can easily show that if $s \neq 0$ is even and $1 / 2<H \leq 1$, then $\nu_{s, H}<0$, see [18], p. 257 (this is based on convexity of the function $\eta \rightarrow \eta^{2 H}$ for $H>1 / 2$ ). This fact simply means that $H$-fractional Brownian process on $\mathbb{S}^{1}$ does not exist in this range. It follows that it does not exist on any $\mathbb{S}^{n}$ since the latter contains $\mathbb{S}^{1}$.

On the other hand, we can easily construct Brownian field (with $H=1 / 2$ ) by letting $f(\eta)=\pi^{1 / 2} 1_{[0, \pi]}(\eta)$ in (12.3). By Bernstein argument it follows that fractional Brownian process exists for all $H<1 / 2$. We have no more explicit representation for it.

For more information about random fields on compact symmetric spaces other than the sphere, we refer to [2].

\section{OpEN PROBLEMS}

In this section we point some open questions.

- Find a closed (explicit) expression of the (upper and lower) spectrum of the fractional Lévy motion $W^{(H)}$, $0<H<1 / 2$.

- Although we know that $H$-fractional Lévy Brownian field does not exist when $H>1 / 2$, find for these $H$ some natural (e.g. appearing in some limit theorems) fields with stationary increments $X$ on hyperbolic space such that $\mathbb{E}|X(z)-X(0)|^{2} \approx d(z, 0)^{H}$ as $z \rightarrow 0$.

- Find the class of the stationary fields generated by the action of the group $S U(1,1)$ on the space of the geodesics (may be every field can be obtained in this way?). Find the spectra of such fields.

- Give a white noise representation $W_{\eta}^{H}=W\left(A_{\eta}\right)$ for $H$-fractional Lévy Brownian fields, $H<1 / 2$, on the hyperbolic space and on the sphere as done by Takenaka [37] in Euclidean case ( $c f$. Sect. 8.1). The 
only construction we are aware of was proposed recently by Estrade and Istas. It handles the case of onedimensional sphere.

- Check whether fractional Ornstein-Uhlenbeck field with covariance $K\left(\eta, \eta^{\prime}\right)=\exp \left\{-a d\left(\eta, \eta^{\prime}\right)^{\alpha}\right\}$ exists for some $a>0, \alpha>1$.

- Investigate the algebraic properties of spectral representations. For example:

- find the spectrum of the stationary field corresponding to the covariance function $K(z)=\omega_{s_{1}}(r) \omega_{s_{2}}(r)$, where $0<s_{1}, s_{2}<\infty$ with $s_{1}, s_{2} \in\left[0, \frac{n-1}{2}\right] \cup\left\{\frac{n-1}{2}+\mathrm{i} \lambda, \lambda \in \mathbb{R}\right\}$.

- Let $\left(X_{z}, z \in \mathbb{D}\right)$ be a field with stationary increments starting at 0 with the structure function $\mathfrak{D}(z)=$ $\mathbb{E}\left|X_{z}\right|^{2}$. Then $\mathfrak{D}(\cdot)$ is a function of negative type. Let $F: \mathbb{R}^{+} \rightarrow \mathbb{R}$ be a Bernstein function. Then $F(\mathfrak{D}(\cdot))$ is a function of negative type, too. Construct a field with stationary increments $\left(Y_{z}, z \in \mathbb{D}\right)$ such that $F(\mathfrak{D}(z))=\mathbb{E}\left|Y_{z}\right|^{2}$. Given the spectrum of $X$ find that of $Y$.

Let us briefly explain the relation between the two problems. Imagine we know the spectral measures $\left\{\nu_{s_{1}, s_{2}}\right\}$ corresponding to covariances $\left\{\omega_{s_{1}} \cdot \omega_{s_{2}}\right\}$. For any covariances $K_{1}$ and $K_{2}$ and the corresponding spectral measures $\mu_{1}, \mu_{2}$ introduce a "convolution" measure

$$
\mu_{1} \otimes \mu_{2}:=\iint \nu_{s_{1}, s_{2}} \mu_{1}\left(\mathrm{~d} s_{1}\right) \mu_{2}\left(\mathrm{~d} s_{2}\right)
$$

The covariance corresponding to $\mu_{1} \otimes \mu_{2}$ is

$$
\begin{aligned}
\iiint \omega_{s_{3}}(\eta) \nu_{s_{1}, s_{2}}\left(\mathrm{~d} s_{3}\right) \mu_{1}\left(\mathrm{~d} s_{1}\right) \mu_{2}\left(\mathrm{~d} s_{2}\right) & =\iint \omega_{s_{1}}(\eta) \omega_{s_{2}}(\eta) \mu_{1}\left(\mathrm{~d} s_{1}\right) \mu_{2}\left(\mathrm{~d} s_{2}\right) \\
& =K_{1}(\eta) K_{2}(\eta)
\end{aligned}
$$

By iterating this argument we see that if $\mu$ corresponds to $K(\cdot)$, then $\mu^{\otimes m}=\mu \otimes \mu \otimes \cdots \otimes \mu(m$ times $)$ corresponds to $K(\cdot)^{m}$. Moreover, the spectral measure

$$
\exp _{\otimes}\{\gamma \mu\}:=\sum_{m=0}^{\infty} \frac{\gamma^{m}}{m !} \mu^{\otimes m}
$$

clearly corresponds to covariance $\exp (\gamma K(\cdot)\}$ and to the structure function

$$
\begin{aligned}
\mathfrak{D}_{\gamma}(\eta): & =2(\exp (\gamma K(0))-\exp (\gamma K(\eta))) \\
& =2 \exp (\gamma K(0))(1-\exp (-\gamma(K(0)-K(\eta)))) \\
& =2 \exp (\gamma K(0))\left(1-\exp \left(-\gamma \mathfrak{D}_{K}(\eta) / 2\right),\right.
\end{aligned}
$$

where $\mathfrak{D}_{K}(\cdot)=2(K(0)-K(\cdot))$ is the structure function related to $K$.

Finally, take a Bernstein function

$$
F(u)=\int_{0}^{\infty}\left(1-\mathrm{e}^{-\gamma u / 2}\right) \nu(\mathrm{d} \gamma)
$$


and observe that

$$
\begin{aligned}
F\left(\mathfrak{D}_{K}(\eta)\right) & =\int_{0}^{\infty}\left(1-\mathrm{e}^{-\gamma \mathfrak{D}_{K}(\eta) / 2}\right) \nu(\mathrm{d} \gamma) \\
& =\int_{0}^{\infty} \frac{1}{2} \exp (-\gamma K(0)) \mathfrak{D}_{\gamma}(\eta) \nu(\mathrm{d} \gamma) \\
& =\int_{0}^{\infty} \exp (-\gamma K(0)) \int\left(1-\omega_{s}(\eta)\right) \exp _{\otimes}(\gamma \mu)(\mathrm{d} s) \nu(\mathrm{d} \gamma) \\
& =\int\left(1-\omega_{s}(\eta)\left[\int_{0}^{\infty} \exp (-\gamma K(0)) \exp _{\otimes}(\gamma \mu) \nu(\mathrm{d} \gamma)\right](\mathrm{d} s)\right.
\end{aligned}
$$

which gives the spectral representation for $F\left(\mathfrak{D}_{K}\right)$.

Acknowledgements. We are much indebted to the referees for careful reading of the manuscript and for their numerous advice that helped us to improve the exposition.

\section{REFERENCES}

[1] J.W. Anderson, Hyperbolic Geometry, 2nd edition. Springer Undergraduate Mathematics Series, Springer-Verlag London Ltd., London (2005).

[2] R. Askey and N.H. Bingham, Gaussian processes on compact symmetric spaces. J. Probab. Theory Relat. Fields 37 (1976) $127-143$.

[3] S. Barsky, Surface texture using photometric stereo data: classification and direction of illumination detection. J. Math. Imaging Vis. 29 (2007) 185-204.

[4] J. Bretagnolle, D. Dacunha-Castelle and J.-L. Krivine, Lois stables et espaces L ${ }^{\mathrm{P}}$. Ann. Inst. Henri Poincaré, Ser. B. 2 (1965/66) 231-259.

[5] J.W. Cannon, W.J. Floyd, R. Kenyon and W.R. Parry, Hyperbolic geometry, in Flavors of Geometry, edited by S. Levy. Cambridge University Press, Cambridge. Math. Sci. Res. Inst. Publ. 31 (1997) 59-115.

[6] N.N. Chentsov, Lévy Brownian Motion for several parameters and generalized white noise. Theory Probab. Appl. 2 (1957) $265-266$.

[7] N.N. Chentsov and E.A. Morozova, P. Lévy's random fields. Theory Probab. Appl. 12 (1967) 153-156.

[8] M. Clerc and S. Mallat, Estimating deformations of stationary processes. Ann. Stat. 31 (2003) 1772-1821.

[9] J.L. Clerc, J. Faraut, M. Rais, P. Eymard and R. Takahashi, Analyse Harmonique. Les Cours du CIMPA (1980).

[10] J.-L. Dunau and H. Senateur, Characterization of the type of some generalizations of the Cauchy distribution, in Probability measures on Groups IX. Oberwolfach (1988). Lect. Notes Math. 1379 (1989) 64-74.

[11] J. Faraut and K. Harzallah, Distances hilbertiennes invariantes sur un espace homogène. Ann. Inst. Fourier (Grenoble) 24 (1974) 171-217.

[12] R. Gangolli, Positive definite kernels on homogeneous spaces and certain stochastic processes related to Lévy's Brownian motion of several parameters. Ann. Inst. Henri Poincaré Sect. B (N.S.) 3 (1967) 121-226.

[13] J. Garding, Shape from texture and contour by weak isotropy. Artif. Intell. 64 (1993) 243-297.

[14] R. Godement, Introductions aux travaux de A. Selberg, Séminaire Bourbaki (1957) 95-110.

[15] I.S. Gradshteyn and I.M. Ryzhik, Table of Integrals, Series, and Products, VI edition. Academic Press, New York (2000).

[16] S. Helgason, Differential Geometry, Lie Groups and Symmetric Spaces, 2nd edition. Academic Press 80 (1978).

[17] S. Helgason, Groups and Geometric Analysis, edited by American Mathematical Society, Providence, RI. Mathematical Surveys and Monographs 83 (2000). Integral geometry, invariant differential operators, and spherical functions. Corrected reprint of the 1984 original.

[18] J. Istas, Spherical and hyperbolic fractional Brownian motion. Electron. Comm. Probab. 10 (2005) 254-262 (electronic).

[19] J. Istas, On fractional stable fields indexed by metric spaces. Electron. Comm. Probab. 11 (2006) 242-251 (electronic).

[20] J. Istas, Manifold indexed fractional fields. Preprint (2009).

[21] N.L. Johnson and S. Kotz, Distributions in statistics: continuous multivariate distributions. Wiley Series in Probability and Mathematical Statistics, John Wiley \& Sons Inc., New York (1972).

[22] P. Lévy, Processus Stochastiques et Mouvement Brownien, 2éme édition, edited by J. Gabay (1965).

[23] E.H. Lieb and M. Loss, Analysis, Graduate Studies in Mathematics, 2nd edition. American Mathematical Society, Providence, RI 14 (2001).

[24] M.A. Lifshits, On the representation of Lévy fields by indicators. Theory Probab. Appl. 24 (1980) 629-633.

[25] M.A. Lifshits, Gaussian Random Functions. Kluwer Academic Publishers (1995). 
[26] H.P. McKean, Brownian Motion with a several-dimensional time. Theory Probab. Appl. 8 (1963) 335-354.

[27] G.M. Molchan, On some problems concerning Brownian motion in Lévy's sense. Theory Probab. Appl. 12 (1967) $682-690$.

[28] G.M. Molchan, On homogenious random fields on symmetric spaces of rank 1 (Russian). Teor. Veroyatnost. $i$ Mat. Statist. (1979) 123-147. Translated in: Theor. Probab. Math. Statist. (1980) 143-168.

[29] G.M. Molchan, Multiparametric Brownian motion on symmetric spaces. VNU Sci. Press, Utrecht (1987). Prob. Theory and Math. Stat. II. Vilnius (1985) 275-286.

[30] G.M. Molchan, Multiparameter Brownian motion (Russian). Teor. Veroyatnost. i Mat. Statist. (1987) 88-101. Translated in: Theor. Probab. Math. Statist. (1988) 97-110.

[31] G.M. Molchan, Private communication (2009).

[32] A.G. Robertson, Crofton formulae and geodesic distance in hyperbolic spaces. J. Lie Theory 8 (1998) $163-172$.

[33] W. Rudin, Fourier Analysis on Groups. Wiley Classics Library, John Wiley \& Sons Inc., New York (1990). Reprint of the 1962 original, A Wiley-Interscience Publication.

[34] L.A. Santaló, Integral geometry on surfaces of constant negative curvature. Duke Math. J. 10 (1943) 687-709.

[35] R. Stanton and P.Thomas, Expansions of spherical functions on non-compact spaces, Acta Math. 40 (1978) $251-276$.

[36] D.W. Stroock, The Ornstein-Uhlenbeck process in a Riemanian manifold, in Proc. of ICCM'98 (Beijing, 1998), First International congress of Chinese Mathematicians. AMS (2001) 11-23.

[37] S. Takenaka, Integral-geometric construction of self-similar stable processes. Nagoya Math. J. 123 (1991) 1-12.

[38] S. Takenaka, I. Kubo and H. Urakawa, Brownian motion parametrized with metric space of constant curvature. Nagoya Math. J. 82 (1981) 131-140.

[39] N.A. Volodin, Some classes of spherically symmetric distributions. Stability problems for stochastic models (Russian) Sukhumi (1987), Vsesoyuz. Nauchno-Issled. Inst. Sistem. Issled., Moscow (1988), Translated in J. Soviet Math. 57 (1991) 3189-3192, $4-8$.

[40] A.M. Yaglom, An Introduction to the Theory of Stationary Random Functions. Revised English edition, Prentice-Hall Inc., Englewood Cliffs, N.J. (1962) 